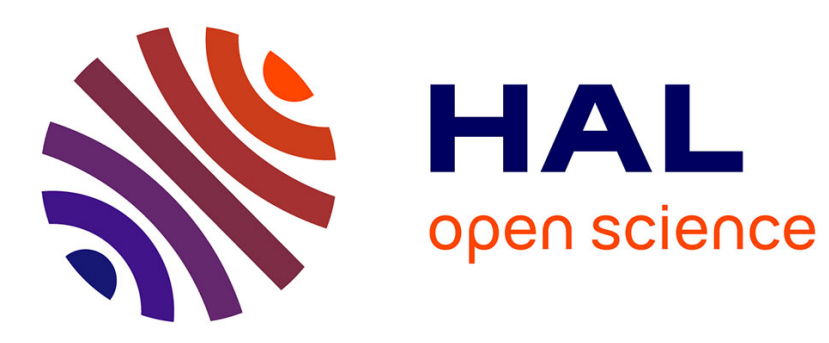

\title{
Advanced characterization techniques based on luminescence in XLPE and modified XLPE
}

G. Teyssedre, Christian Laurent, Bo Qiao

\section{To cite this version:}

G. Teyssedre, Christian Laurent, Bo Qiao. Advanced characterization techniques based on luminescence in XLPE and modified XLPE. Cross-linkable Polyethylene based blends and nanocomposites, Springer, Chap. 6, pp. 99-157, 2021, 978-981-16-0486-7. hal-03008898

\section{HAL Id: hal-03008898 \\ https://hal.science/hal-03008898}

Submitted on 17 Nov 2020

HAL is a multi-disciplinary open access archive for the deposit and dissemination of scientific research documents, whether they are published or not. The documents may come from teaching and research institutions in France or abroad, or from public or private research centers.
L'archive ouverte pluridisciplinaire HAL, est destinée au dépôt et à la diffusion de documents scientifiques de niveau recherche, publiés ou non, émanant des établissements d'enseignement et de recherche français ou étrangers, des laboratoires publics ou privés. 


\title{
Advanced characterization techniques based on luminescence in XLPE and modified XLPE
}

\author{
Gilbert Teyssèdre ${ }^{\left(1^{*}\right)}$, Christian Laurent ${ }^{(1 * *)}$ and Bo Qiao ${ }^{\left(2^{* *}\right)}$ \\ ${ }^{(1)}$ Laboratory on Plasma and Energy Conversion - Laplace, \\ University of Toulouse and CNRS, Toulouse (31062), France \\ ${ }^{(2)}$ Key Laboratory of Luminescence and Optical Information, Ministry of Education \\ Institute of Optoelectronics Technology, Beijing Jiaotong University, Beijing (100044), P.R .China \\ *gilbert.teyssedre@laplace.univ-tlse.fr \\ **christian.laurent@laplace.univ-tlse.fr \\ ***boqiao@bjtu.edu.cn
}

\begin{abstract}
Crosslinked Polyethylene finds application in the insulation of high voltage cables, constituting a high demanding domain in terms of electrical performances. High quality XLPE grades are now produced for insulation under high voltage direct current (HVDC) stress. Luminescence techniques have constituted original techniques along the development of such materials, particularly as regards the role of defects and residues in the behavior of materials in terms of electrical charges stabilization. Luminescence provides a family of extremely sensitive techniques, though limited to substances with unsaturated groups: in materials like XLPE only additives, residues and defects are optically active. After recalling the grounds of luminescence principles in organic materials, we explain the techniques implemented, mainly based on photoluminescence and on an analysis of optical emissions related to charge traps into materials. The main results obtained with the identification of the role of these 'defects' are presented before addressing the knowledge brought by luminescence methods, including electroluminescence, on thermal and electrical ageing aspects of polyethylene materials.
\end{abstract}

Keywords: XLPE, luminescence, crosslinking, cable insulation

This chapter is the final version as a contribution (Chap 6) to an upcoming book on Cross linkable Polyethylene based blends and nanocomposites, Edited by Prof. Sabu Thomas and Prof. Zakiah Ahmad, Springer (2020). 


\section{I-Introduction}

\section{Contents}

\section{II-Luminescence from materials}

\section{II.1 Definitions}

II.2 Absorption and emission phenomena

II.3 Luminescence from polymers

II-3-1-Photoluminescence

II-3-2-Luminescence due to charge recombination

II-3-3-Other forms of luminescence

II-3-4-Correlation between luminescence and analytical techniques

\section{III-Implementation of luminescence measurements}

III.1 Materials

III.1.1-Standart XLPE

III.1.2-Models of XLPE compounds

III.1.3-Grafted XLPE

III.2. Multipurpose luminescence set-up

III-2-1-Outlook of the characterization system

III.2.2-Detection systems

III-2-3-Luminescence techniques according to excitation means

\section{IV-Luminescence in XLPE and XLPE compounds}

IV-1-Base line for luminescence properties: LDPE base resin vs. XLPE

IV-I-1-LDPE base resin

IV-I-2-XLPE and thermally-treated XLPE

IV-I-3-Overall: LDPE vs. XLPE

IV-2-Model compound for interpretation of XLPE deep trapping sites

IV-2-1-Luminescence signature of model compounds: by-products of cross-linking reactions

IV-2-2- Luminescence signature of XLPE model compounds with grafted species

IV-2-3- Luminescence signature of model compounds: anti-oxidant and its derivatives

IV-3-Investigating cable XLPE insulation with screen diffusing species

IV-3-1-Influence of storage duration of XLPE slices in the presence of SC

IV-3-2-Fluorescence and phosphorescence vs. cable radius in unaged and aged samples

IV-3-3-Identification of photoluminescence contributions

\section{V-Diagnosis, degradation and ageing processes}

$\mathrm{V}$-1-Electroluminescence from LDPE and XLPE

$\mathrm{V}$-1-1-Rationale for EL measurements

$\mathrm{V}-1-2-$ Field regimes of light emission - AC vs DC EL

V-1-3-Electroluminescence spectra

V-2-Cathodoluminescence as a prototype source for interpreting EL spectra

$\mathrm{V}-2-1-T h e \mathrm{CL}$ spectra from polyolefins

$\mathrm{V}$-2-2-The $\mathrm{CL}$ spectrum decomposition into different processes

$\mathrm{V}$-3-Ageing diagnosis

V-3-1-EL yield of aged materials

$\mathrm{V}-3-2-\mathrm{PL}$ from thermally degraded materials

V-3-3-Contact sensitization

\section{VI-Conclusions}




\section{I-Introduction}

Crosslinked Polyethylene (XLPE) is used for several decades now as insulation in high voltage buried or submarine power transmission cables, i.e. rated above $60 \mathrm{kV}$ [1]. The major advantages of XLPE over the competitive technology based on traditional oil-impregnated paper insulation are lower dielectric losses, less complicated installation procedure and maintenance, and no risk of leakage implying improved respect for environment. The technical risks of XLPE-insulated high voltage cables could be a lack of knowledge of the degradation withstanding ability of the insulation. Today's hot topics are more related to the extension of its application to HVDC energy transport. Significant progresses have been achieved in adapting the material formulation to the new challenges brought by the form of stress to be supported. Space charge processes and conduction processes are two properties that had to be seriously addressed for ensuring the reliability of cables under high voltage direct current stresses. These issues have been addressed in a number of recent reviews and we won't go into more details here [2] [3].

Failure in transmission has always important consequences related to the time for energy outage and repairing costs. While some failures may arise from isolated events as natural disasters or human mistakes with digging for example, the repetition of middle term failure due to intrinsic weakness of the cable under continuous application of nominal stress is really a catastrophic aspect. In this perspective, the aim of the European project named ARTEMIS [4] was to investigate degradation processes and derive diagnostic properties for ageing evaluation and modelling of high voltage XLPE cables. The progresses in producing even safer cables have been achieved with ever improving extrusion processes in cables as well as cleanliness of rough materials used in HV cable production.

XLPE has therefore some high end niches in which high quality materials must be produced. Materials quality in the cable industry is naturally being assessed with electrical characterization as impedance spectroscopy, conductivity measurements, breakdown measurements, space charge measurements, etc in one hand, mechanical properties and thermal stability on the other hand. XLPE became at the top of cable insulation materials firstly because of its excellent thermomechanical properties [5]. The improvement of thermomechanical properties was achieved with compounding materials: peroxide crosslinking and protection against oxidation with introducing antioxidants is the way it is generally achieved. Because of this compounding, XLPE is a material containing, besides defects in the main chain, by-products, being volatile or not, and small amounts of residues. In turn, these moieties impart most of the differences in electrical properties compared to low density polyethylene for example. There has been a need for characterization techniques capable of making the link between chemicals on the one hand, and their impact on the behavior of electrical charges in the insulation, in the other hand. Indeed, depending on excitation means, different relaxation mechanisms can be at play such as the relaxation of optically excited groups, the recombination of charges of opposite polarity coexisting in the same region, or relaxation following hot carrier processes (impact excitation or ionisation).

Luminescence techniques have appeared as original characterisation tools in this perspective, the reason why we have dedicated one chapter on its principles and results in this book. Depending on the excitation means, luminescence features may provide information related to chemicals, or to the interaction between electrical charges and groups acting as deep traps. These techniques can therefore be considered as at the frontier between physico-chemical and electrical characterisation tools.

Before going into the details, let's mention a few specificities of luminescence methods when applied to materials such as XLPE:

-Ideally, polyethylene does not contain unsaturations in its main chain, and then no luminescence should be detected in the uv-vis domain. Then, the response necessarily comes from additives, residues, defects, ageing by-products, etc being dispersed into the material. Both extrinsic (additives, crosslinking byproducts) and intrinsic (oxidised groups, insaturations) chromophores are potentially emitting groups in XLPE materials. 
-Luminescence can be very sensitive in presence of optically active moieties. However, moieties can be present without being luminescent. Therefore, it cannot pretend to cover all the possible defects;

-In a solid material, many energy exchange phenomena can be at play, the response of a molecule can be dependent on its environment and therefore identifying the luminescence bands is not as straightforward as it can be for infrared spectroscopy for example.

In the next section, we first present the general aspects of luminescence phenomena, particularly in the case of organic solids. In section 3, we present the kind of XLPE materials that have been considered in luminescence experiments, how it was processed, and we describe the methods that were implemented.

Then, the results are presented in two main sections related to luminescence from compounds and to ageing and degradation aspects viewed from the luminescence standpoint. 


\section{II-Luminescence from materials}

\section{II-1-Definitions}

Luminescence is the light emitted by a substance when the excitation is not due to heat, by opposition to incandescence. The excited states responsible for material luminescence can be produce by different excitation sources [6], among them: photon absorption (photoluminescence PL) [7], chemical reactions (chemiluminescence $\mathrm{CHL}$ ) [8], recombining charges (recombination induced luminescence RIL) [9] [10], electric field application (electroluminescence EL) [11] [12], electron beam irradiation (cathodoluminescence CL or electron-beam induced luminescence EBIL) [13] [14] [15], temperature (thermoluminescence TL) [16] [17] [18], etc. PL, CHL, RIL, EL, EBIL, TL refer to the source of excitation itself which can generate a series of elementary excitations contributing to the luminescence. As example, photon absorption can raise excited states of atoms/molecules but also can ionize them. The luminescence will result from relaxation of neutral excited states and from charge recombination in case of ionization. Another example is thermoluminescence where the thermal energy is used to detrap electrical charges, the luminescence deing due to subsequent charge recombination. The generic term used to name a form of luminescence does not imply a single elementary process. Common luminescence mechanisms typical in polymeric materials are given in Table 1.

\begin{tabular}{c|c}
\hline \hline $\begin{array}{c}\text { Luminescence type } \\
\text { Photoluminescence } \\
\text { PL }\end{array}$ & $\begin{array}{c}\text { Promotion of excited state } \\
\text { CHL }\end{array}$ \\
\hline $\begin{array}{c}\text { Chemiluminescence } \\
\text { (mild UV) }\end{array}$ \\
\hline RIL & $\begin{array}{c}\text { Chemical reaction } \\
\text { (typical: oxidation of polyolefins) }\end{array}$ \\
\hline Recombination induced luminescence & $\begin{array}{c}\text { Recombination between trapped carriers } \\
\text { (no kinetic energy) }\end{array}$ \\
\hline Electroluminescence & $\begin{array}{c}\text { Mixture of excitation processes } \\
\text { induced by electric field }\end{array}$ \\
\hline Electron beam induced luminescence EBIL & $\begin{array}{c}\text { Hot electron impact and processes } \\
\text { induced by irradiation }\end{array}$ \\
\hline Plasma-Induced Luminescence & $\begin{array}{c}\text { Luminescence following excitation of the surface } \\
\text { by a plasma discharge }\end{array}$ \\
\hline Thermoluminescence & $\begin{array}{c}\text { Luminescence in a temperature ramp following } \\
\text { excitation of the surface by a ionizing radiation or } \\
\text { other way of depositing charges }\end{array}$ \\
\hline \hline
\end{tabular}

Table 1. Overview of common luminescence types in insulating polymers

\section{II.2 Absorption and emission phenomena in organic molecules}

\section{II-2-1-Absorption}

The absorption of UV-VIS radiations in organic molecules is restricted to functionnal groups called chromophores that have valence electrons of weak energy. Under excitation conditions, electrons located on the orbitals $\sigma$ (simple covalence bonds), $\pi$ (multiple covalence bonds) and $n$ (lone electron pair) will be promoted to anti-bonding orbital (excited state) $\pi^{*}$ or $\sigma^{*}$. Transitions $\sigma \rightarrow \sigma^{*}$ and $\pi \rightarrow \pi^{*}$ have a strong energy, whereas transitions $\mathrm{n} \rightarrow \sigma^{*}$ and $\mathrm{n} \rightarrow \pi^{*}$ have a weak energy. $\mathrm{n}$ electrons do not form bonds and they don't have anti-bonding orbitals [19] [20]. The possible electronic transitions upon mild UV absorption are schematically represented in Figure 1. 


\section{a) $\sigma \rightarrow \sigma^{*}$ transitions}

One electron located on simple covalent bond is excited to the corresponding anti-bonding orbital. The energy gap between the two states is strong. As example, methane which has only $\mathrm{C}-\mathrm{H}$ bonds, can only stands $\sigma \rightarrow \sigma^{*}$ transitions corresponding to a maximum in absorption at $125 \mathrm{~nm}$ which cannot be observed in classical UV spectroscopy (200 $\mathrm{nm}$ to $300 \mathrm{~nm})$.

\section{b) $\mathbf{n} \rightarrow \sigma^{*}$ transitions}

Compounds containing atoms with lone electron pairs (oxygen, nitrogen) are able of such transitions of lower energy. They can be promoted by photons of wave length between $150 \mathrm{~nm}$ and $250 \mathrm{~nm}$.

\section{c) $n \rightarrow \pi^{*}$ and $\pi \rightarrow \pi^{*}$ transitions}

Optical spectroscopy of most organic compounds is based on the electronic transitions from $\mathrm{n}$ or $\pi$ state to the excited state $\pi^{*}$. The presence of unsaturated groups in the molecule is therefore needed to provide $\pi$ electrons. One can quote a strong transition $\pi \rightarrow \pi^{*}$ in the far UV for the molecules containing $\mathrm{C}=\mathrm{C}$ groups. In contrast, $\mathrm{n} \rightarrow \pi^{*}$ transitions have a weak intensity but classical in carbonyls spectroscopy.

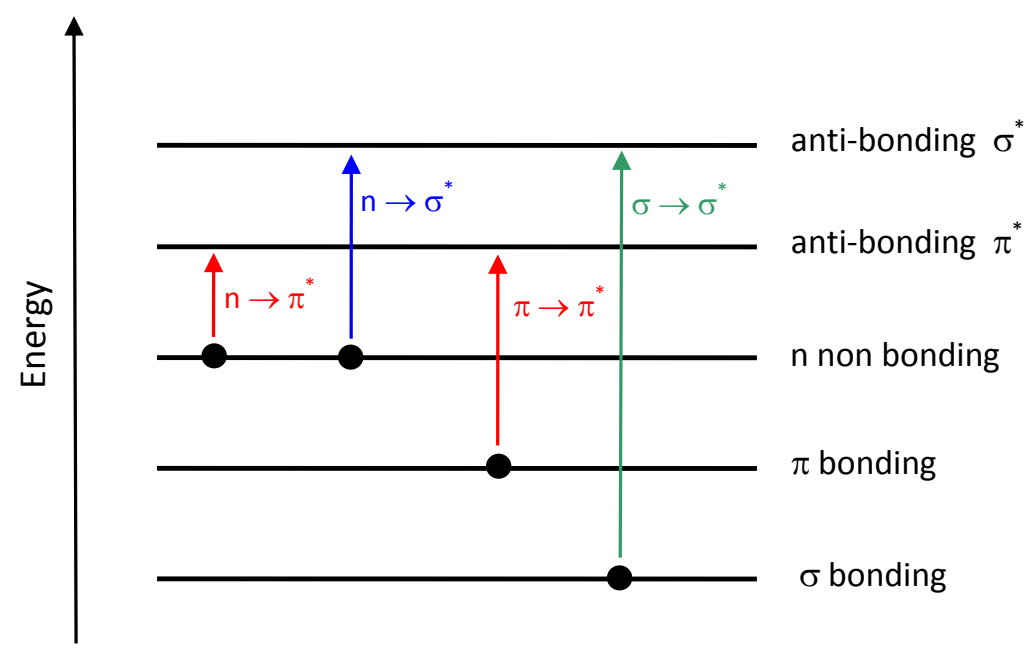

Figure 1. Possible electronic transitions in organic molecules in the UV-VIS spectrum

\section{II-2-2-Emission}

The electronic state of most organic molecules can be separated in singlet $(\mathrm{S})$ and triplet states (T). Difference between these two types is based on spin coupling -see Figure 2 . In singlet state, two electrons of the same energy state have a coupled spin (anti-parallel spin) whereas in triplet state, they have a parallel spin [19].

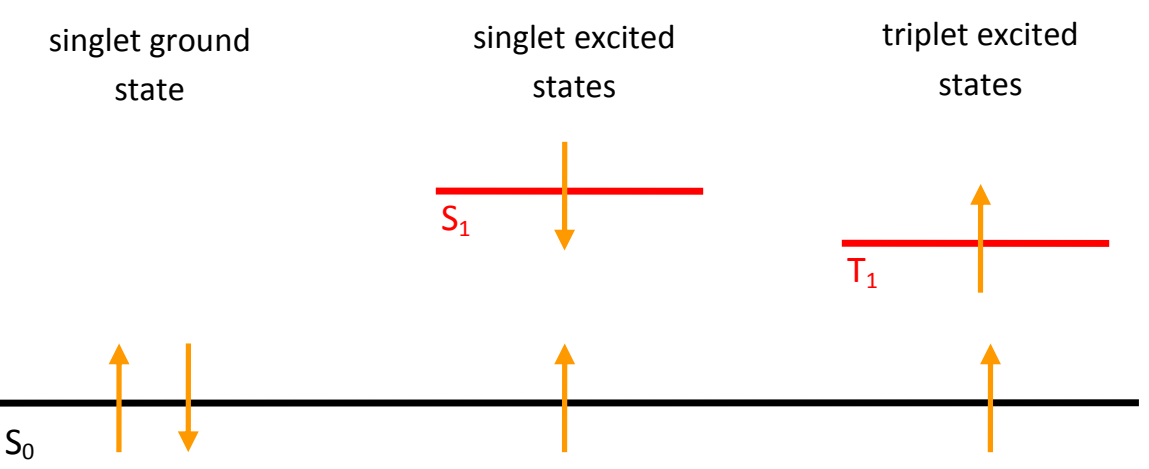

Figure 2. Spin coupling in ground and excited states defining singlet and triplet excitons 
The energy conversion in a molecule can be approached with reference to the Jablonsky representation (Figure 3) [20].

\section{d) Energy absorption (transition 1 in Figure 3)}

The molecule absorbs some energy raising an electron from the ground state to an excited state with vibrationnal and rotationnal components.

\section{e) Energy relaxation (transitions 2, 5 and 6 in Figure 3)}

The molecule can relax following radiative and non radiative pathways.

In non radiative relaxation, the energy is dissipated by internal conversion (transitions 2) through collision with other atoms/molecules, or by intersystem crossing (transition 4). In the later, the electron spin is reversed and the molecule lies in a lower energy triplet state.

In radiative relaxation, the excited electron loses its energy going from the first excited state to the ground state. This can happen from the first excited singlet state (fluorescence) or from the first excited triplet state (phosphorescence). The life time of a triplet state is much longer (up to several s) than the one of a singlet state (order of ns) because it is a spin forbidden transition. Moreover, non radiative transfers compete with radiative relaxation from a triplet state in such a way that phosphorescence is usually observed only at low temperature which hinders inhibition by another atom/molecule.

1. absorption

2. vibrationnal relaxation

3. internal conversion

4. inter-system crossing

5. fluorescence

6. phosphorescence
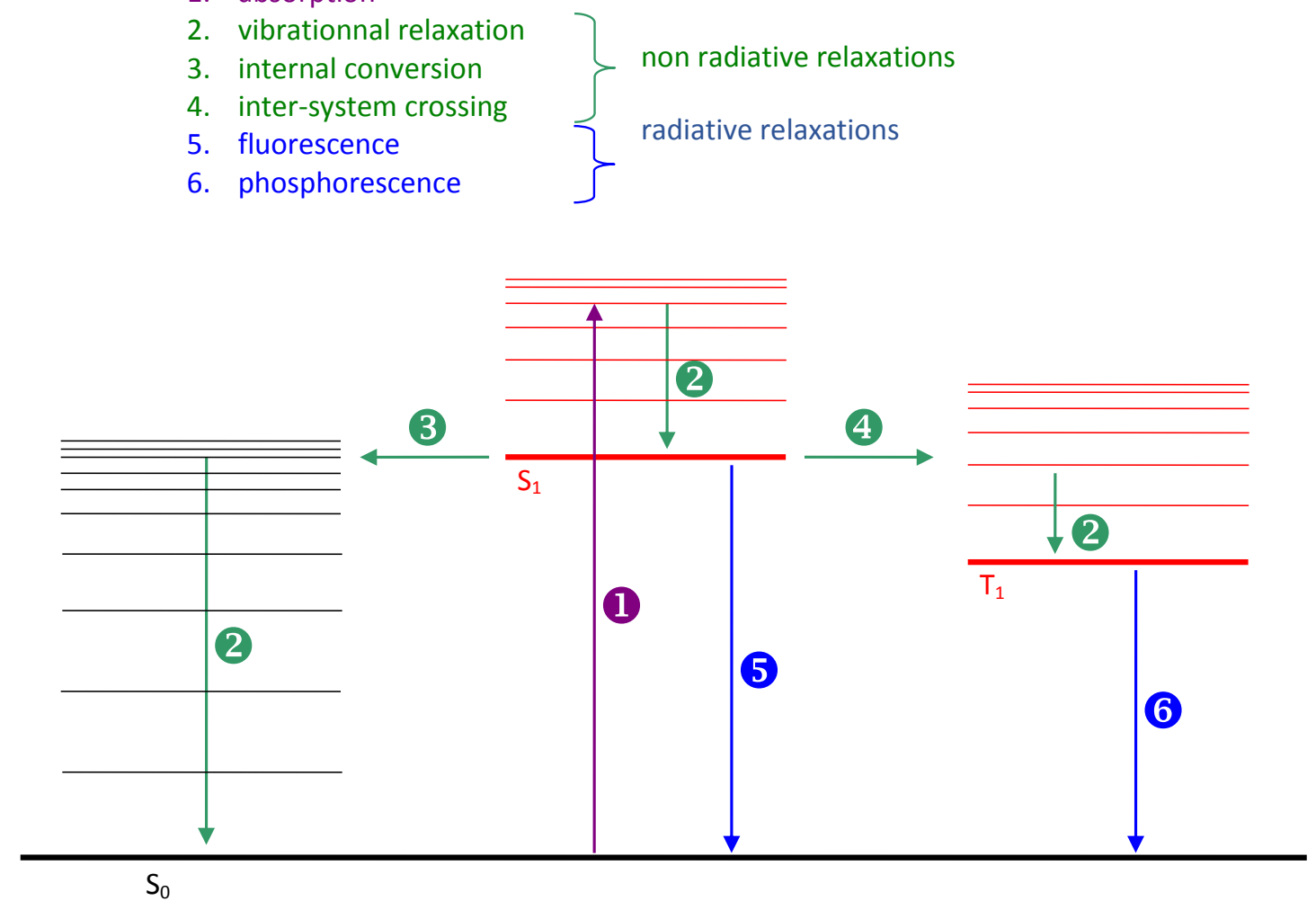

Figure 3. Absorption, fluorescence and phosphorescence spectra of a small organic molecule and the corresponding energy state diagram with radiative and non radiative transitions in a Jablonsky representation. 


\section{II.3 Luminescence from polymers}

The emission of light by a substance following an optical excitation is called photoluminescence which is a common spectroscopic technique in the field of materials even if its interpretation is not straightforword in the case of polymers [6]. Two kinds of polymers have to be distinguished depending on the chromophores they contain. In a first kind, the chromophores are not belonging to the monomer unit and can be bonded (unsaturation of the hydrocarbon chain) or not (impurities or residues of cross-linking in XLPE) to the macromolecular chain. In a second kind, chromophores are present in the monomer units (as in PET or PEN). This distinction is important as the interpretation of the emission spectra will not be as straightforward in the first type when compared to the second type.

Both emission and excitation spectra are usually recorded in photoluminescence spectroscopy. If the former is straightforward, the latter is less common: the frequency of the excitation light is varied, and the luminescence is monitored at the typical emission frequency of the material being studied.

\section{II-3-1-Photoluminescence}

In photoluminescence, the emitted light has a lower energy and therefore longer wavelength than the excitation light. The time between absorption and emission may vary from femtoseconds to milliseconds or even to minutes or hours under special circumstances. The relative short and relative long time between absorption and emission lead to fluorescence and phosphorescence respectively.

There is another peculiarity of polymer photoluminescence, which is due to the existence of interaction between neighbor molecules. The emitting species can be different from the excited ones, and excited states can be form upon the interaction of several atoms (excimers and exciplexes). Quenching processes are also of particular importance.

\section{a) Fluorescence}

Fluorescence occurs when an orbital electron of a molecule, atom or nanostructure releases back to its ground state keeping the same parity. The relaxation is fast (order on ns).

$$
\begin{array}{lc}
\text { Excitation process: } & \mathrm{S}_{0}+\mathrm{hv}_{\mathrm{ex}} \rightarrow \mathrm{S}_{1} \\
\text { Light emission process: } & \mathrm{S}_{1} \rightarrow \mathrm{S}_{0}+h v_{\mathrm{em}}+\text { heat }
\end{array}
$$

Here, hv is photon energy with h (Planck's constant) and v (frequency of the light). State $S_{0}$ is the ground state while state $S_{1}$ is its first excited singlet state.

\section{b) Phosphorescence}

Phosphorescence occurs when an orbital electron of a molecule, atom or nanostructure releases back to its ground state after spin reversal. The relaxation is much longer /fluorescence, order of ms to some s. Hence phosphorescent materials can "store" absorbed energy for a certain time.

$$
\begin{array}{lc}
\text { Excitation process: } & \mathrm{S}_{0}+\mathrm{hv}_{\mathrm{ex}} \rightarrow \mathrm{S}_{1} \\
\text { Transition process: } & S_{1} \rightarrow T_{1} \\
\text { Light emission process: } & \mathrm{T}_{1} \rightarrow \mathrm{S}_{0}+\mathrm{hv}_{\mathrm{em}}+\text { heat }
\end{array}
$$

Here, hv, $S_{0}, S_{1}$, and heat mean the same as above, while $T_{1}$ is a triplet state.

During the phosphorescence process, the material is excited to higher energy states, then undergo an intersystem crossing into energy state of higher spin multiplicity, usually triplet states, $S_{1} \rightarrow T_{1}$, as seen in 
Figure 3. The transition $\mathrm{T}_{1} \rightarrow \mathrm{S}_{0}$ is normally a spin-forbidden transition, which is why the lifetime of triplet states (phosphorescence) is longer than that of singlet states (fluorescence).

\section{c) Excimers and exciplexes}

In the excited state, energy transfer between monomeric units of a single chain, or between units of different chains, give birth to specific excitation called excimers and exciplexes. In the former case, an excited state of a chromophore is coupled to a groud state of another identical chromophore. Experimentally, this gives birth to a broader emission band, without apparent structure, with a weaker energy than the single chromophore emission. Another form of complex is the exciplexe, which results from the coupling between an excited molecule and a different molecule in its ground state. These complexes can be at the origin of delayed fluorescence when two excited molecules are in a triplet state. In this case, a complex can be form between the two molecules $\mathrm{M}$ with subsequent relaxation to a singlet excited state and a ground state according to:

$$
{ }^{\mathrm{T}} \mathrm{M}^{*}+{ }^{\mathrm{T}} \mathrm{M}^{*} \longrightarrow{ }^{\mathrm{S}}(\mathrm{MM})^{*} \longrightarrow{ }^{\mathrm{S}}(\mathrm{M})^{*}+\mathrm{M}
$$

In certain circunstances, the fluorescence is delayed in time far beyond its natural life time.

\section{d) Inhibition (quenching)}

This is an important phenomenon where a species can interact with an excited state inhibiting thereby the luminescence. Quenchers are usually heavy atoms or paramagnetic molecules. A current process is the quenching of the luminescence of a molecule $\mathrm{M}$ by an oxygen molecule (triplet ground state), according to:

$$
{ }^{\mathrm{S}} \mathrm{M}^{*}+{ }^{\mathrm{T}} \mathrm{O}_{2} \longrightarrow{ }^{\mathrm{S}} \mathrm{M}+{ }^{\mathrm{s}} \mathrm{O}_{2}
$$

\section{II-3-2-Luminescence due to charge recombination}

The luminescence induced by charge recombination (RIL) is the light emitted by the material after its irradiation by an ionizing source (x-rays, $\gamma$-rays, electron beam, plasma source, etc.) [16] [18] [21] [22] [23] [24] [25] [26] [27]. The phenomenon of RIL is general and can be unraveled in most of the polymeric materials [10] [27]. During the irradiation, or a short time after (typically $<1 \mathrm{~s}$ ), different mechanisms can contribute to the emission like direct excitation of the molecules without ionization, or immediate recombination between electrons (or anions) and holes (or cations). Recombination-induced luminescence dominates afterwards where the thermal fluctuations (isothermal luminescence) control the detrapping of charges and subsequent recombination with the recombining centers. Thermoluminescence is due to the same effect of charge detrapping and recombination due to the thermal energy provided to the polymer during heating.

In part IV of the present chapter, we will intensively use the emission spectra in charge recombination-induced luminescence to infer the nature of recombining centers in XLPE. The recombination regime is obtained in Plasma-Induced Luminescence, or PIL, where a polymer film is put in contact with a cold plasma [25]. Some details of the analysis are given below.

\section{a) Charge recombination regime after contact with a cold plasma: isothermoluminescence}

Here we illustrate the phenomenon by taking the example of luminescence analysis in XLPE films after contact with a cold plasma of helium at low pressure [7]. Further information on the techniques is given in section III-23. A typical example of the kinetic of the luminescence is shown in Figure $\mathbf{4}$ where the overall signal is fitted to three different luminescence components : a first one is due to the photoluminescence of the PE sample excited by the UV of the discharge, with time constant corresponding to the lifetime of triplet states; a second one is due to chemiluminescence -see paragraph II-3-3 [8], and a third one is due to charge recombination with a typical kinetic, giving a total light decay in time of the form: 


$$
\mathrm{I}(\mathrm{t})=\mathrm{I}_{01} \exp \left(-t / \tau_{1}\right)+\mathrm{I}_{02} \exp \left(-t / \tau_{2}\right)+\mathrm{I}_{03}(1+\alpha \mathrm{t})^{-\mathrm{m}}
$$

where $\mathrm{I}$ is the luminescence amplitude, $\mathrm{I}_{\mathrm{0} i}$ are pre-exponential factors, $\mathrm{t}$ is the time elapsed from the end of the plasma treatment, $\alpha$ and $m$ are adjustable coefficients and $\tau_{i}$ are time constants. The order of magnitude of $\alpha$ is $0.1 \mathrm{~s}^{-1}$ and $\mathrm{m} \approx 1$. In the example shown in Figure $4 \alpha$ is large; hence the last term is of the form $\mathrm{I}_{3} \cdot \mathrm{t}^{-\mathrm{m}}$.

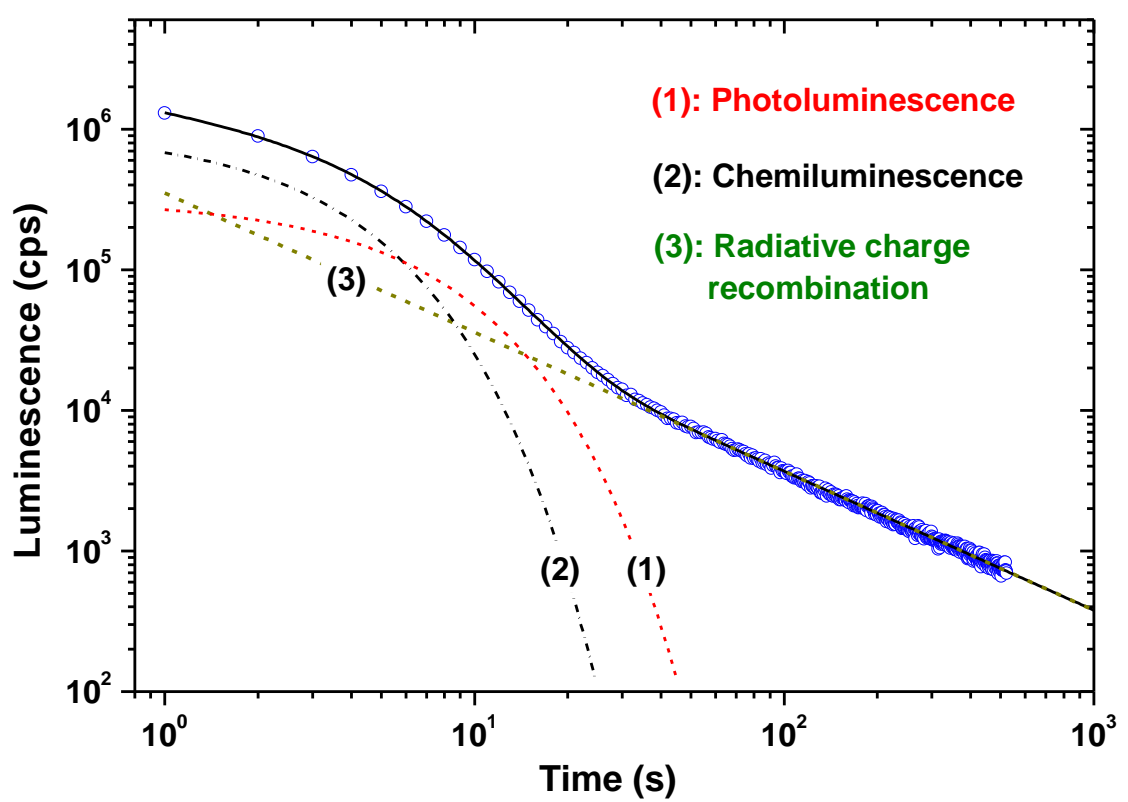

Figure 4. Kinetic of isothermoluminescence of XLPE after contact with a cold plasma and its contributing phenomena. Adapted from [7]

The reciprocal time dependence of the light amplitude can be assigned to electron tunneling to positively charged luminescence centers [28] [29] [30] [31]. Such kinetic analysis allows to define a time window where recombination is the sole contribution and therefore to isolate the emission spectrum of the process.

\section{b) Charge recombination regime after contact with a cold plasma: thermoluminescence}

Figure 5 shows the Thermoluminescence (TL) curve recorded for crosslinked polyethylene after the end of an isothermoluminescence record while heating at a linear rate of about $5^{\circ} \mathrm{C} / \mathrm{min}$. The curve in blue was obtained after correction from the temperature dependence of the main emission derived from temperature dependent photoluminescence experiments [7]. Two transition regions appear at about $-120^{\circ} \mathrm{C}$ and $-60^{\circ} \mathrm{C}$. It is tempting to associate these with the $\gamma$ and $\beta$-relaxations of polyethylene which occur in the respective temperature regions according to dynamical mechanical analysis [32]. Overall, the luminescence is due to charge detrapping following recombination. The emission spectrum is the same as in isothermal RIL experiments.

\section{II-3-3-Other forms of luminescence}

\section{a) Chemiluminescence}

Chemiluminescence is the light emitted during a chemical reaction. It is a well-known phenomenon in polyolefins where oxidation reactions lead to the formation of $\mathrm{C}=\mathrm{O}$ bonds. The light is generated during the last step of chemical reaction from $\mathrm{C}=\mathrm{O}^{*}$ to $\mathrm{C}=\mathrm{O}$ with a typical signature at $415 \mathrm{~nm}$ [33]. Chemiluminescence has been deeply investigated in our group along thermal degradation of polyolefins, both through the kinetics of the integral emission and its spectrum that changes as a function of the reaction [25] [27] [33] [34] [35] [36] [37] [38]. 


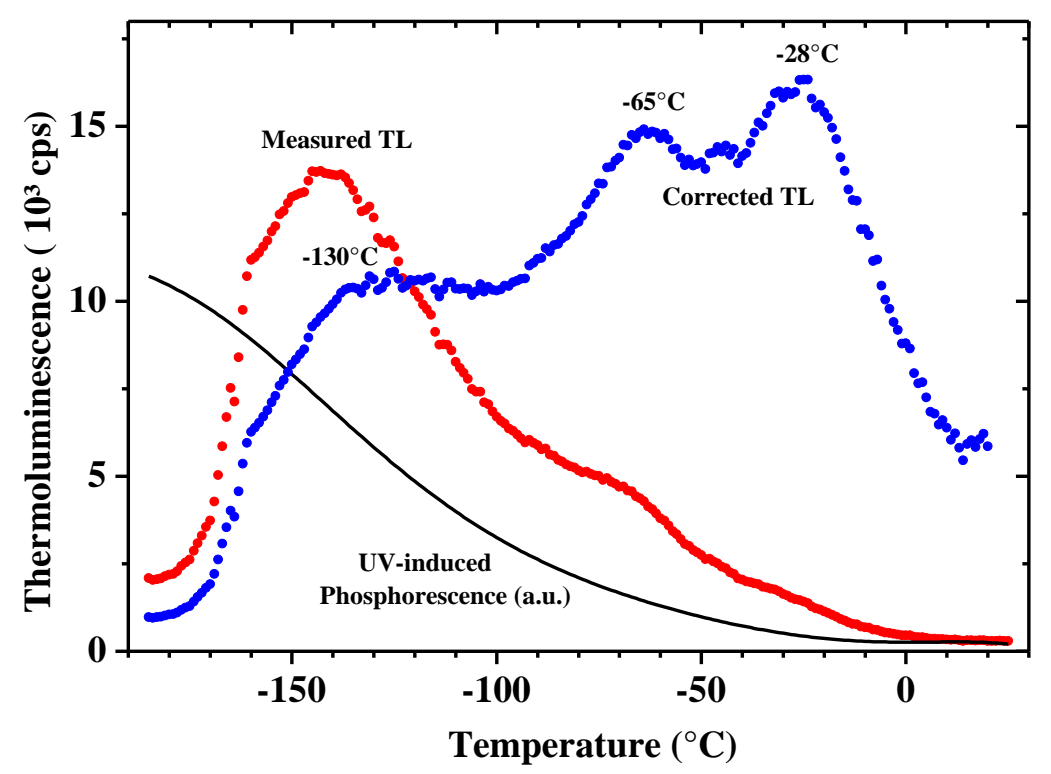

Figure 5. Thermoluminescence curve obtained after excitation of crosslinked polyethylene by a plasma discharge. The luminescence is corrected from the temperature dependence of the main emission at about $450 \mathrm{~nm}$ in photoluminescence experiments [7].

\section{b) Cathodoluminescence (or electron beam induced luminescence)}

Cathodoluminescence is the light emission following excitation of the matter by an electron beam [39]. Exposure of a solid to an electron beam with several $\mathrm{keV}$ in energy induces a number of processes in the specimen, which lead to the formation of secondary and backscattered electrons, and generate also characteristic radiation and X-ray, and Auger electrons. The secondary electrons generate electron-holes pairs which can recombine directly or be trapped, and further released to excite the luminescence by recombination. It is noteworthy that the energy of excitation considerably exceeds the bandgap width of any material. The excitation of luminescent centers can occur not only via direct excitation, but also as a result of radiative and non-radiative transitions from higher lying energy states. As a consequence, $\mathrm{CL}$ spectra often demonstrate a larger amount of emission bands than other luminescence spectra.

\section{c) Electroluminescence}

Electroluminescence is the light emitted by a material put under electric stress. It is a well-known process in any media. It was firstly reported in 1963 [40] in organic semiconductors and exploited as organic light emitting devices. On the other hand, electroluminescence in insulating polymers was firstly reported in 1967 [41]. In these large band gap materials, the excitation mechanisms can be related to the kinetic energy of the charges, or their potential energy. In the former case, injected charges of a few electron-volts kinetic energy can impact excite some centers. In the later, recombining charges can be the source of the light. Mechanisms involved in EL of insulating materials are still under debate [42] and complex phenomena are involved as will be see in Section V.

\section{II-3-4-Correlation between Luminescence and analytical techniques}

As a general analytical method for polymers, luminescence spectroscopy suffers from the same problem as UVvisible absorption spectroscopy: the level of information obtained is coarse because of the close similarity in energy of the electronic transitions and their vibronic progressions, in which substituent effects are either absent or not resolved. The technique has been particularly powerful, however, in detecting trace amounts of oxidation products [43]. 
Different reasons make it not as a classical analytical method for characterizing solid polymers. First, luminescence techniques can be very sensitive on compounds having high yield, and can be relatively unsensitive for non-luminescent dyes. So it makes a first difference with other methods like Infrared Spectroscopy in which any molecule in principle provides absorption. Second, luminescence suffers from a lack of database of reference spectra for given chemical groups, mainly because the signature may depend on the environment of the molecule. It is therefore not easy to precisely identify chemical groups based only on their luminescence properties Thirdly, in solid state notably, it is extremely delicate to pretend being quantitative, because of the many deexcitation and transfer routes that can be followed by excited states.

Though luminescence in solids is not developed as an ordinary physico-chemical characterization technique, several works tentatively established correlations between luminescence, particularly chemiluminescence, and other analytical techniques [44]. Techniques as Electron Spin (or Paramagnetic) Resonance - ESR or EPR [45] or FTIR emission [46] are interesting as they provide signal linked to active groups during reactions. Rychly et al have published works in favour for decomposition of hydroperoxides as being responsible for the light emission during oxidation of Polypropylene [47]. However, the correlation is not necessarily straightforward. For example, Blakey and George [46] demonstrated through FTIR emission that the chemiluminescence intensity was proportional to the accumulation of carbonyl species formed during oxidation, rather than the rate of oxidation, which is predicted from classical chemiluminescence mechanisms associated with auto-oxidation. Kron et al correlated the amount of peroxide contained in polypropylene, as determined by iodometry, and the amount of light being detected under thermoluminescence curve in inert atmosphere. [48] A good correlation between ESR and CL as peroxyl radical detectors has been found under mechanical stress, though the level of information in the reaction is not the same [49].

All of these works are based on integral light and do not use the spectral distribution of the light for bringing understanding. For a qualitative comparaison of information brought by FTIR and photoluminescence, one may consider Figure 6 with phosphorescence spectra of benzophenone (a) [50] compared to that of XLPE grafted with a benzophenone derivative (b) [10]. Carbonyl derivatives have intense phosphorescence from their ${ }^{3}$ ( $n \rightarrow$ $\pi^{*}$ ) states. Phosphorescence spectra of most aromatic carbonyl compounds show a common feature as regards their vibrational structure. The vibronic structure observed in the spectra corresponds to relaxation to vibronic levels of the ground level. The vibronic structure of acetophenone phosphorescence emission was perfectly resolved at $4 \mathrm{~K}$, and the strongest bands have been identified as harmonics of the $v(\mathrm{C}=\mathrm{O})$ Raman band at 1684 $\mathrm{cm}^{-1}$ [51]. At $100 \mathrm{~K}$, the energy difference between structured peaks is less due to overlapping of several vibronic states [7]. Because of this broadening introduced by vibrational levels, broad bands are obtained and identification of emitting groups is not direct.

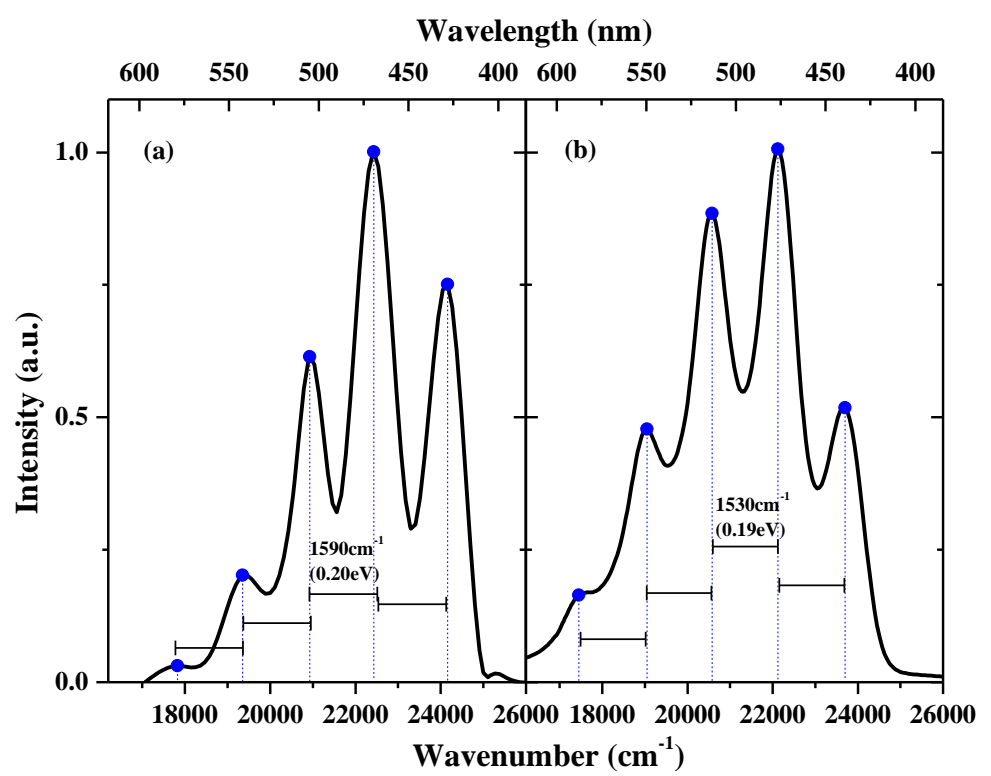

Figure 6. Phosphorescence spectra of benzophenone (a) in ethanol [50] and of XLPE grafted with benzophenone protonoxy (b). [10]. The averaged vibronic structure is of $1590 \mathrm{~cm}^{-1}$ in (a) and $1530 \mathrm{~cm}^{-1}$ in (b). 
Besides, luminescence may have extraordinary and unique features as with electroluminescence, which as we shall see in the following, provides signatures of degradation products being formed, in quantities that are not detectable with other methods specially when it is mixed with a great number of other chemicals in low concentration. In (electrical) ageing we must admit that there are no detectable new chemicals being formed (cf. ARTEMIS project [4]), due to field application whereas EL could be detected. We also showed that chemicals being quinoline derivatives, that have been proved to diffuse from semiconducting screens in Medium Voltage cables, were not detected by any other analytical method applied in the same project on the same samples.

\section{III-Implementation of luminescence measurements}

\section{III.1 Materials}

As state above, hydrocarbons made of aliphatic bonds do not emit light in the visible domain. For being emissive, unsaturation must be present. A first family of defects is those existing in low density polyethylene as depicted in Figure 7. Jacques and Poller [52] investigated model compounds of these defects by photoluminescence and this study guided the interpretation of fluorescence from LDPE.

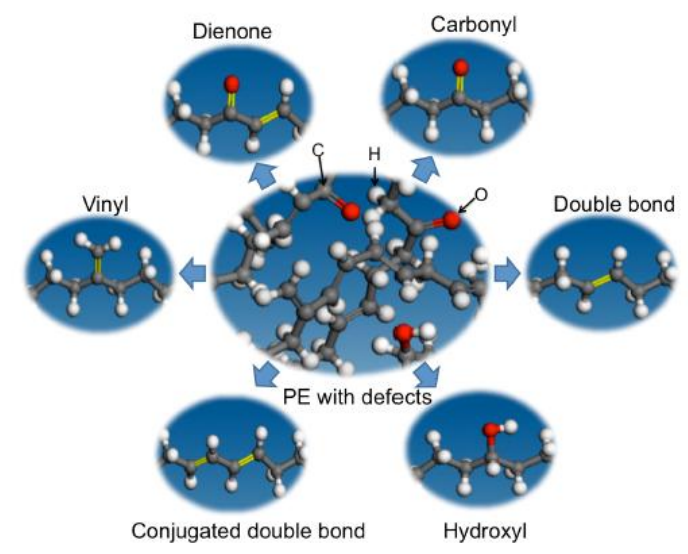

Figure 7. Six typical chromophore structures that may occur in the carbon chain of Polyethylene: carbonyl, dienone, hydroxyl, double bond, conjugated double bond and vinyl. Taken from [53]

A second set of defects is that provided by additives (antioxidant) and residues due to the crosslinking process. Examples are given in Figure $\mathbf{8}$ in the case of dicumyl peroxide chemically cross-linked PE. Compared to in-chain defects presented above, residues do not have chemical bonds to the PE chains and can therefore migrate and be expelled from the polymer. Therefore, particular care has to be taken about sample handling and storage as the amount of by-products evolves in time. In some cases, outgasing of the sample at $60^{\circ} \mathrm{C}$ is achieved to force by-products expelling and probe a 'dried' material. We will show that there can be traces of by-product detected in the luminescence response.
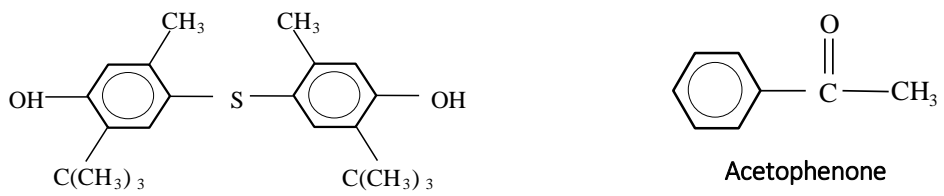

Anti-oxidant<smiles>C=C(C)c1ccccc1</smiles>

$\alpha$-methylstyrene<smiles>CC(C)(O)c1ccccc1</smiles>

Cumylalcohol

Figure 8. Chemical structure of possible additives and residues of DCP-chemically cross-linked PE [54] 


\section{III-1-1-Standard XLPE}

All of the luminescence methods applied to polymers can be applied to materials in film form. An advantage of film in respect to powder or pellets is that the excitation and the amount of probed material are well controlled. Results presented herein were obtained on two kinds of samples.

1) Pressmolding and peroxide crosslinking in a single run. Like for cable poduction, pellets containing expected amounts of peroxide and of antioxidants, of the order of $1 \mathrm{wt} . \%$ each, are first molded under pressure at temperature slightly above the melting point, then the temperature is increased to $190^{\circ} \mathrm{C}$ to perform the crosslinking. The sample is then progressively cooled down to room temperature. Caution along this process is with molding material: to make unmolding easy, polymer films are layed onto the mold. However, it was shown that when using PET as protecting film, specific luminescence signatures could be produced. The second caution is about the release of by products: to control this amount, the cooling and the environment of samples should be kept the same. The sample thickness of typically $100 \mu \mathrm{m}$ is a good balance. Making thiner films with repeatable and homogenous thickness can be problematic. Making thicker films means higher voltage needs to be applied when carrying out recombination-induced luminescence or electroluminescence experiments.

2) The second way of making films is by peeling from high voltage cables. Provided high quality knife can be used, films of good surface quality can be produced. In the frame of the Artemis project performed on aged cables, such peelings were produced within the $15 \mathrm{~mm}$ thick insulation: continuous rolls of $8 \mathrm{~cm}$ width, about $15 \mathrm{~m}$ in length and $150 \mu \mathrm{m}$ in thickness were analyzed with different electrical and nonelectrical method, including luminescence [4]. An advantage was that the rolls reflected the position of samples along the cable radius. Also, compared to press-molded samples, peeled samples tend to retain better the by-products. An important result was obtained in this study of photoluminescence spectra versus position along the radius of the cable. It concerns the diffusion of antioxidant from the semiconducting screens of the cable into the insulation. This was reported in a pair of papers and further commented later on [55] [56].

\section{III-1-2-Models of XLPE compounds}

On the one hand, XLPE is a rich material in term of residues compared to thermoplastic materials. On the other hand, there is a lack of tabulated data on spectral features of potential chemicals involved in luminescence. To isolate these signatures, selected compounds were incorporated into LDPE. It concerns the main crosslinking by-products, acetophenone, cumyl alcohol and alpha-methylstyrene, an antioxidant, (4, 4'-thiobis (2-terbutyl-5methylphenol), AO-), and some of its known derivatives once it has reacted with oxygen [57] [58] as presented in Table 2. The results about this study will be commented in section IV. The way samples were realized is by mixing $0.1 \mathrm{wt} . \%$ of the products with LDPE and pressmolding the pellets at $130^{\circ} \mathrm{C}$ [57].

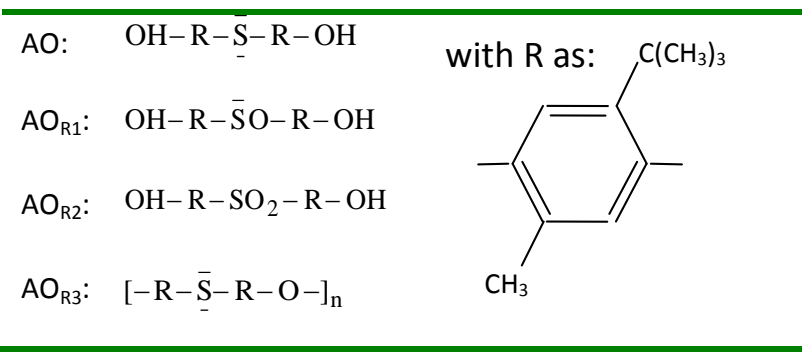

Table 2. Chemical formula of the antioxidant 4, 4'-thiobis (2-terbutyl-5-methylphenol) and its reaction products. 


\section{III-1-3-Grafted XLPE}

The last set of samples investigated was XLPE with grafted groups on it [10]. The project participated in the general objective to develop anti-treeing compounds, often called voltage arresters, for XLPE insulation. These aromatic groups, styrene and benzophenone derivative notably, cf. Figure $\mathbf{9}$ are supposed to easily catch the energy provided by hot carriers and prevent material ionization. The modification process is patented [52]. We shall see that groups can be strongly luminescent.<smiles>[R]CCCC(C)CC(=O)Oc1ccc(C(=O)c2ccccc2)cc1</smiles>

grafted benzophenone crotonoxy

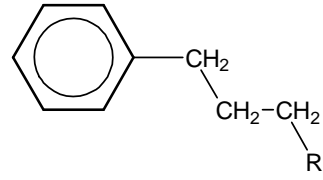

grafted styrene

Figure 9. Chemical structures of grafted groups on XLPE [10]

\section{III-2-Multipurpose luminescence set-up}

The available luminescence commercial systems are mainly for PL detection, which can not meet the requirement of luminescence measurement of insulating polymers. In addition, commercial apparatuses tend to be dedicated to probe liquid solutions more than solid and they often don not apply to large samples. Collectiong light from a large surface can be a solution when working with low intensity luminescence signal. For all of these reasons, we have developed a multi-purpose chamber for light detection.

\section{III-2-1-Outlook of the characterization system}

The chamber that has been developped is depicted in Figure 10. A specificity of the installed system is to detect faint light intensity and to be efficient, for photoluminescene excitation, in the UV region (from $220 \mathrm{~nm}$ and on) when commercial spectrofluorimeters operate beyond $280 \mathrm{~nm}$. The system contains five main elements:

(1) excitation source system (electric field, electron beams, photons, and plasma);

(2) optical detecting system (PM system and CCD camera system);

(3) optical path controlling system;

(4) temperature controlling system;

(5) pumping system.

The excitation source system and the testing system are two core parts for luminescence measurements, while the other systems are auxiliary components. The testing system also contains three parts according to the optical axes:

-Optical axis $\mathrm{N}^{\circ} 1$ for photoluminescence excitation;

-Optical axis $\mathrm{N}^{\circ} 2$ with a cooled photomultiplier (PM) working in photon counting mode for integral light detection;

-Optical axis $\mathrm{N}^{\circ} 3$ for a grating dispersive system (4.5 $\mathrm{nm}$ in resolution) coupled to a liquid nitrogen cooled charge coupled device (CCD) camera for spectral analyses which covers the wavelength range from $230 \mathrm{~nm}$ to $840 \mathrm{~nm}$. 


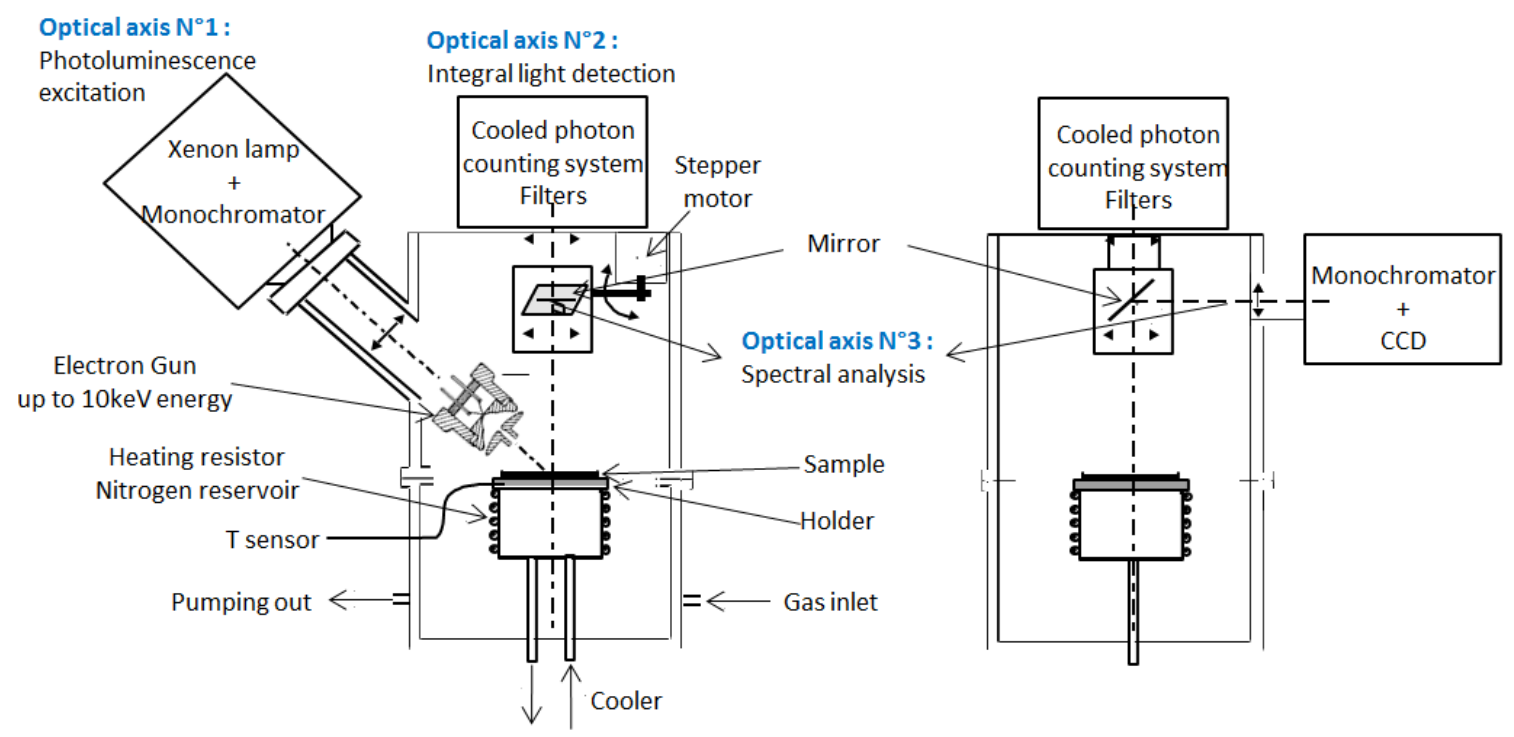

Figure 10. Experimental setup used for multi-purpose luminescence detection.

The mechanical environment is a light proof dark chamber connected to a turbo-molecular double pumps system to achieve high vacuum, up to $10^{-7} \mathrm{mbar}$. High vacuum is used in electroluminescence measurements in order to avoid gaseous discharges during measurements. Samples can be placed on the holder which contact to a heating resistor, a reservoir than can receive liquid nitrogen or a thermostated liquid, and a temperature sensor, to control the temperatures from liquid nitrogen temperature up to $\approx 150^{\circ} \mathrm{C}$ (in atmosphere only). The multi-purpose chamber is designed to accommodate different kinds of luminescence excitation as described below. Some key parts of the set-up will be introduced below.

\section{III-2-2-Detection systems}

The photomultiplier (PM), type of Hamamatsu R943-02, is used to count photons emitted by the material. It allows recording resolved luminescence in time. It operates at a controlled temperature of $-30^{\circ} \mathrm{C}$ by a Peltier cooling system. This temperature has the effect of reducing the thermal emission of electrons from the photocathode, which implies extremely low noise (a few counts per second). The spectral response of the photocathode of the photomultiplier is from 210 to $900 \mathrm{~nm}$; it is relatively flat in the wavelength range from 300 to $900 \mathrm{~nm}$.

The signal transmitted by the PM is pre-amplified and then passes through a set of amplifier/discriminator and is finally transferred to a computer via a card of "fast" acquisition pulse counter from ORTEC. The integration time (dwell time) is variable from micro-seconds to several minutes. We use the PM signal recording for lifetime estimation in photoluminescence, or integral light variation as a fonction of time or stress for example for field threshold estimation in electroluminescence.

The CCD camera (Charge Coupled Device) from Princeton Instruments (LN /CCD-1100-PB) works at a controlled temperature of $-110{ }^{\circ} \mathrm{C}$. This camera is associated with an imaging spectrograph (type: Jobin-Yvon CP200) which ranges at 200 lines $/ \mathrm{mm}$. It covers a spectral range between $190 \mathrm{~nm}$ and $820 \mathrm{~nm}$. The sensitive part of the camera has a resolution of $1100 \times 330$ pixels, each covering on area of $24 \times 24 \mu \mathrm{m}^{2}$, summing information over the 330 rows. The spectral resolution is 4.5 nanometers. The CCD camera works either in spectral detection mode or in imaging mode. The output of the dispersive system extends over the length of the detector at 1100 pixels, i.e. a point per $0.573 \mathrm{~nm}$. The spectrometer is calibrated periodically using known emission lines of a Xenon lamp.

All the optical coupling is achieved using quartz lenses and mirrors. Band pass filters, 2.5 " in diameter can be interposed along Axis 2 for realizing light detection in given wavelength range. Also, such filters can be put 
along axis 3: it was used for example to make imaging of the EL emission from the surface of samples with filtering in selected wavelength ranges [60].

\section{III-2-3-Luminescence techniques according to excitation means}

\section{a) Optical excitation: Photoluminescence}

During photoluminescence $(\mathrm{PL})$ measurement, the samples mounted into the chamber were excited by a Xenon source coupled to an irradiation monochromator. The excitation beam is with and angle of $40^{\circ}$ in respect to the vertical axis. In this way, the specular reflexion from the excitation beam does not enter the light collecting system. The Xenon lamp has a power of $150 \mathrm{~W}$ from Jobin-Yvon. It is coupled to an excitation monochromator (double pass monochromator HD10UV from Jobin-Yvon with 1200 lines/mm grating). The excitation wavelength varies form $220 \mathrm{~nm}$ to $700 \mathrm{~nm}$ and is set manually. The bandwidth of the irradiation window can be adjusted from $0.2 \mathrm{~nm}$ to $4 \mathrm{~nm}$ using slits. Coupling between the sample, the excitation source, and CCD camera, was achieved by optical path through the quartz lenses window of the chamber. PL measurements are performed at $\approx-130^{\circ} \mathrm{C}$ especially for phosphorescence and at room temperature especially for fluorescence. Prior to testing samples in photoluminescence measurements at low temperature, the chamber is evacuated and filled in with helium at atmospheric pressure. The phosphorescence spectra can be isolated by synchronizing the light excitation switch off of the shutter placed in front of the irradiation source and the exposure to the CCD camera. In this way, fluorescence, which has a very short lifetime, and the excitation beam are not present in the obtained spectra.

There is a high pass filter at $300 \mathrm{~nm}$ in front of the CCD camera in order to cutoff the excitation light of wavelength shorter than $300 \mathrm{~nm}$, which is particularly useful for samples as XLPE where most of the excitation wavelengths are in the range $220-300 \mathrm{~nm}$. The emission spectrum is integrated under different times by the CCD camera; typically 10-20 s integration proves enough to have a good resolution when realizing photoluminescence spectra.

Phosphorescence lifetimes can be estimated using the photomultiplier, by synchronizing excitation shutter switch off and start of pass trigger in the counting board of the PM. Lifetimes down to microseconds can be detected in this way.

\section{b) Electric field excitation: Electroluminescence}

During electroluminescence (EL) measurement, the sample arranged as seen in Figure $\mathbf{1 1}$ was installed in the chamber and put under high vacuum. Samples are provided with semitransparent electrodes of gold, 30nm in thickness or of ITO (Indium Tin oxide). The electrode diameter is typically $50 \mathrm{~mm}$. The two electrodes HV ring electrode (inner diameter $30 \mathrm{~mm}$ ) and ground flat electrode (diameter $40 \mathrm{~mm}$ ) are connected to the high voltage and ground respectively. The ring electrode allows the light emission analyses from the center of the sample.

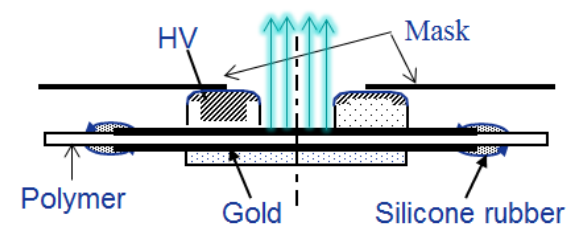

Figure 11. Configuration of EL measurement

Most of the measurements are carried out under secondary vacuum to avoid discharges in the environment of the sample and characterize luminescence only from the solid. Light detection is carried out by two systems PM and CCD as explained above. The integration time with the CCD for EL spectra acquisition is usually longer than for PL, of several minutes, due to a much lower light intensity compared to PL. 
Direct current (DC) or alternating current (AC) stresses are applied to the dielectric in film form. The DC stress is applied using $35 \mathrm{kV}$ power supply from Fug, Germany. DC current is measured simultaneously using a Keithley 617 programmable electrometer. The AC power supply consists of a high voltage amplifier (x2000, up to $20 \mathrm{kV}$ voltage amplifier from Trek, USA) controlled by a function generator. The phase of AC voltage can be synchronized with the counting board of the PM for achieving the phase-resolved EL of the materials.

\section{c) Electron beam excitation: Cathodoluminescence}

During cathodoluminescence $(\mathrm{CL})$ measurement, we use a home-designed electron beam gun mounted into the chamber, cf. Figure 10, providing electrons of up to $10 \mathrm{keV}$ in energy. The filament is at high voltage and the anode at the ground. The distance between the electron gun and the sample on the holder is about $40 \mathrm{~mm}$. The axis of the gun is at $50^{\circ}$ to the normal of the sample plane rendering possible light detection along the normal to the samples. The beam current is about $0.5 \mu \mathrm{A}$. Experiment were also carried out under a vacuum better than $10^{-6} \mathrm{mbar}$, at ambient temperature. Emission spectra and light intensity were recorded for different electron beam energies and for different radiation time with the same beam energy.

\section{d) Plasma silent discharge for recombination-induced luminescence}

In order to charge the surface of the sample we produce a silent discharge obtained between two plane parallel electrodes, Figure 12. The sample is deposited on the lower one and the upper one is made of quartz in such a way that the discharge is initiated between the two dielectrics. The plasma gap of $5 \mathrm{~mm}$ is powered at a frequency of $5.5 \mathrm{kHz}$ under a voltage of about $1.5 \mathrm{kV} \mathrm{rms.}$

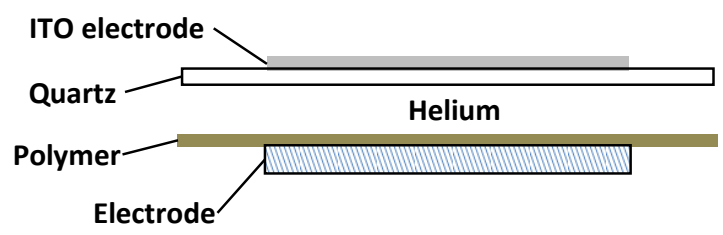

Figure 12. Configuration for recombination induced luminescence measurement

The sample is contacted with a reservoir in which liquid nitrogen circulates: experiments are achieved at low temperature to increase luminescene yield. The discharge is produced in helium at atmospheric pressure. Before introducing the helium, the chamber is pumped down to $10^{-5} \mathrm{~Pa}$. An electronic circuit controls the interaction time of the plasma with the surface. It adjusts the delay between the end of plasma generation and the beginning of measurement. In this way, the luminescence of the polymer surface can be analyzed in situ through the upper transparent electrode. For our purpose, we deal with short plasma interaction times $(\approx 5 \mathrm{~s})$ for which it has been shown that the surface modification is mild. The relaxation of the surface is quite slow and the luminescence can be detected during more than 30 min after plasma interaction at low temperature and for about $5 \mathrm{~min}$ at room temperature. As shown previously, photon counting is achieved with the photomultiplier and spectra are acquired with the CCD in different time intervals after the discharge switch off.

Thermoluminescence can be achieved while heating up the sample after isothermal light decay measurements. 


\section{IV-Luminescence in XLPE and XLPE compounds}

As previously underlined, chemically cross-linked -XL (vs. irradiation cross-linked) PE is a complex material. Based on cross-linkable LDPE (a special PE in which the number of $\mathrm{C}=\mathrm{C}$ is controlled for providing sites for crosslinking) it has all the complexity of the base LDPE (unsaturations, oxidized species, etc. belonging to the chain and anti-oxidant agent as additive to protect the base material from oxidation, plus other additives likely) plus the ones due to the cross-linking process itself, i.e. it contains by-products of cross-linking agent which can be formed as isolated molecules (i.e. non bonded to the main chain) or be strongly bonded to the chain. In addition, by-products of the anti-oxidant are found as species bonded or not bonded to the macromolecular network. All these chemical species can potentially trap electrical charges and therefore are potential candidates as recombination centers for carriers of opposite polarity. Investigation on the luminescence properties of different formulations gives information about the active trapping sites.

\section{IV-1-Base line for luminescence properties: LDPE base resin vs. XLPE}

\section{IV-1-1-LDPE base resin}

Figure 13 shows the luminescence features resulting from PL and RIL experiments on LDPE base resin. A commercial peroxide cross-linkable LDPE base resin was used. As it constitutes the baseline for the overall study and to improve the control on its purity, LDPE powder has been treated in a solution of ethanol before being processed under film form. The objective was to remove extractable impurities due to pollution from the ambient. No antioxidant was used during the molding procedure so as to get the simplest possible reference. The thickness of the film was $150 \mu \mathrm{m}$. The fluorescence of the reference LDPE sample peaks at $335 \mathrm{~nm}$ for short excitation wavelengths (210-245 nm). For longer excitation wavelengths, two bands at $360 \mathrm{~nm}$ and 378 $\mathrm{nm}$ appear as shown in the emission spectrum in Figure 13. These bands are best resolved for an excitation wavelength of $250 \mathrm{~nm}$. At low temperature, a strong phosphorescence emission exceeding the fluorescence yield is detected. The spectrum is broad, structureless, with a maximum peaking at around $458 \mathrm{~nm}$. Note that the fluorescence band is visible at low temperature as a wing in the short wavelength domain. The spectral shape and band positions are consistent with data in the literature [6] [61].Polyolefins usually fluoresce at about 330-340 $\mathrm{nm}$. With increasing oxidation degree, extra bands in the region 360-380 nm have been reported [61], see Figure 14. As the reference material was antioxidant-free, it is not unexpected that oxidation took place in the specific sample under study.

\section{Main luminescence features for LDPE}

- UV-induced fluorescence: peak at $335 \mathrm{~nm}$ for excitation 210-245 nm; two extra-bands at 360 and $378 \mathrm{~nm}$ for excitation at $250 \mathrm{~nm}$. Assigned to carbonyls of the enone type. Strong dependence on the oxidation degree.

- UV-induced phosphorescence: broad band peaking at $458 \mathrm{~nm}$. Assigned to carbonyls of the dienone type.

- RIL: red shifted/UV phosphorescence and peaking at $475 \mathrm{~nm}$. Tentatively assigned to different electronic transitions on phosphorescent species and/or to recombination on higher conjugated phosphorescent species acting as deep traps

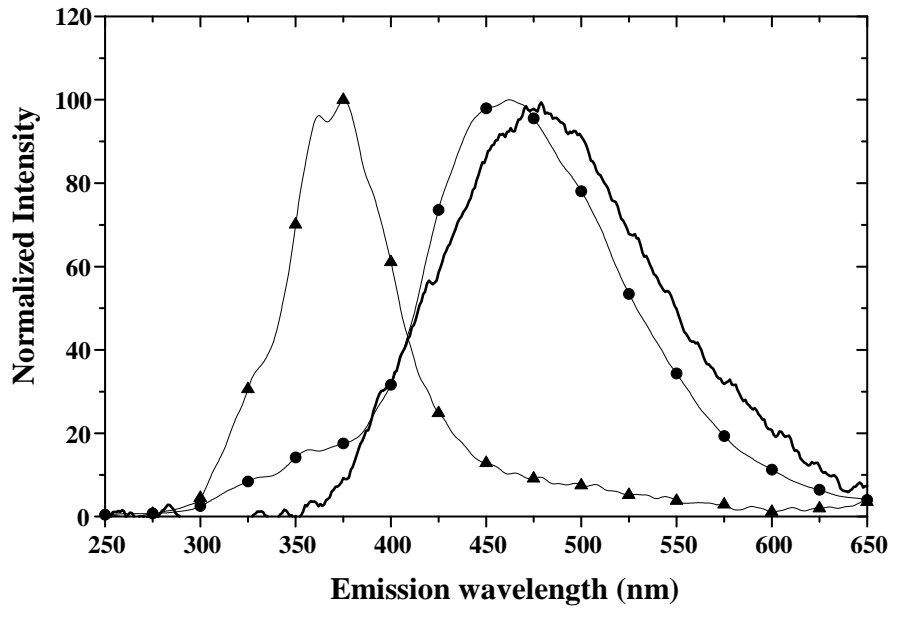

Figure 13. Luminescence features in LDPE base resin tested in film form. Photoluminescence:

$(\boldsymbol{\Delta})$ at RT; (-): at low temperature. $\lambda_{\text {exc }}=250 \mathrm{~nm}$. RIL spectrum (-) [54] 


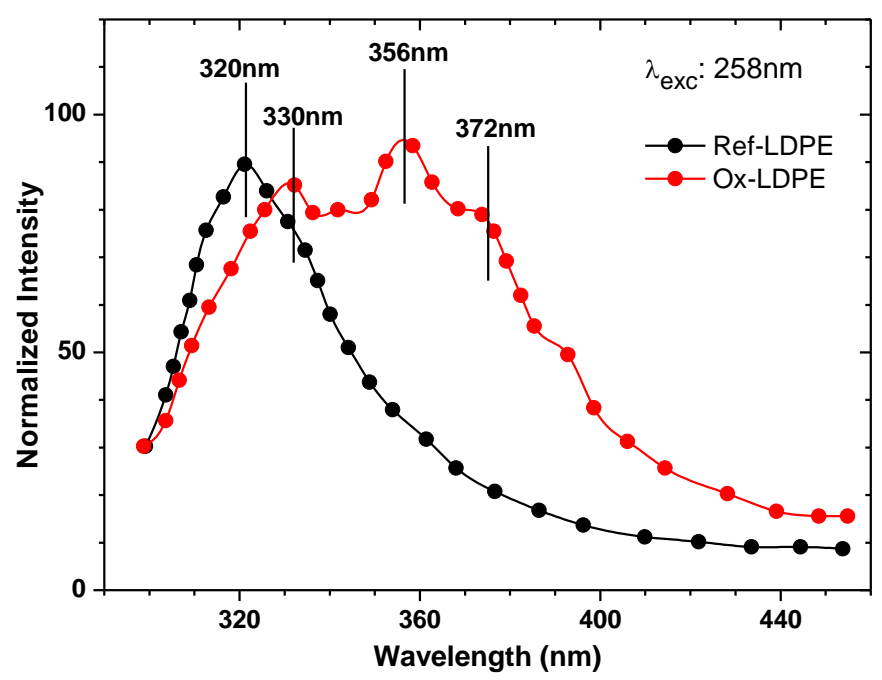

Figure 14. Fluorescence from LDPE: Reference vs. oxidized, with an excitation wavelength of $258 \mathrm{~nm}$. Adapted from [61].

Phosphorescence at around $450 \mathrm{~nm}$ is also a classical feature for such material. So, following data from the literature, the luminescence of LDPE base resin is related to the presence of carbonyls and unsaturated carbonyls of the enone type in the case of fluorescence [62] [52] and of the dienone type for phosphorescence [6] [62], which structure is given below :<smiles>[R]C=CC([R])=O</smiles>

(a)<smiles>[R]C=CC(=O)C=CC([R])=O</smiles>

The RIL spectrum is clearly in the phosphorescence domain. It is shifted towards long wavelengths relatively to the UV-induced phosphorescence spectrum and the maximum intensity is found at around $475 \mathrm{~nm}$. This shift may have two origins: either it corresponds to the involvement of different chromophores, such as longer polyenone sequences, or it results from the excitation of different energy levels of the same chromophore. Carbonyl compounds exhibit both $(n, \pi *)$ and $(\pi, \pi *)$ states, and as $(n, \pi *)$ states are the lowest lying triplets in enone compounds they would be preferentially involved upon charge recombination [9]. Note that they have the lowest ionization potential of the molecule.

\section{IV-1-2-XLPE and thermally-treated XLPE}

Two high voltage cables insulated by XLPE were produced specially for the Artemis project [4]. The insulation thickness was $14 \mathrm{~mm}$. Samples cut from the insulation of these cables are referenced A and B. Another cable with $27 \mathrm{~mm}$-thick XLPE insulation has been introduced in the project for validating the present approach, since it was available in both the unaged and aged forms. Samples from this reference cable are labelled C.

\section{a) Sample handling}

Measurements were performed on rolls cut from the cables, having width $80 \mathrm{~mm}$ and mean thickness $150 \mu \mathrm{m}$ [55]. In order to minimise effects of diffusion of low molecular weight species from the screen, samples corresponding to the region 2 to $4 \mathrm{~mm}$ from the inner screen of the cable were analysed. More details on the effect of sample handling and cutting procedures on the measurements are given elsewhere [4]. A further problem that was considered is the preconditioning of the specimens before any measurement, in order to have reproducible results. It is clear that not all cross-linking by-products will exit the cable during ageing and thus could contribute to degradation processes. On the other hand, it is necessary to prepare samples whose 
properties change little with storage time. Therefore, a preconditioning procedure in which the samples were placed in an oven for $48 \mathrm{~h}$ at $50{ }^{\circ} \mathrm{C}$ in order to expel crosslinking by-products has been adopted. No by-products were detected after such treatment using either HPLC (High Performance Liquid Chromatography) or FTIR (Infrared Spectroscopy) [4]. As the luminescence features of these byproducts are of interest, we have compared results obtained on specimens before and after preconditioning.

\section{b) Fluorescence}

Figure 15.a shows the principal features of the emission spectra obtained from preconditioned samples. Figure 15.b shows the corresponding excitation spectra monitored at the peak wavelength of the components identified in the emission spectra, for preconditioned and non preconditioned samples. The fluorescence excitation spectra -i.e. the emitted light intensity monitored at a given emission wavelength while scanning the excitation wavelength - have been corrected for the wavelength dependence of the excitation power. However, the region $220-230 \mathrm{~nm}$ is probably overestimated. Therefore, these spectra should be considered only for comparison of the relative emission yield of the different materials. The effect of preconditioning is shown on the excitation spectra for cable A, which is known as the material containing the highest content of volatile species, due to extrusion conditions. At least 3 emission bands have been identified:

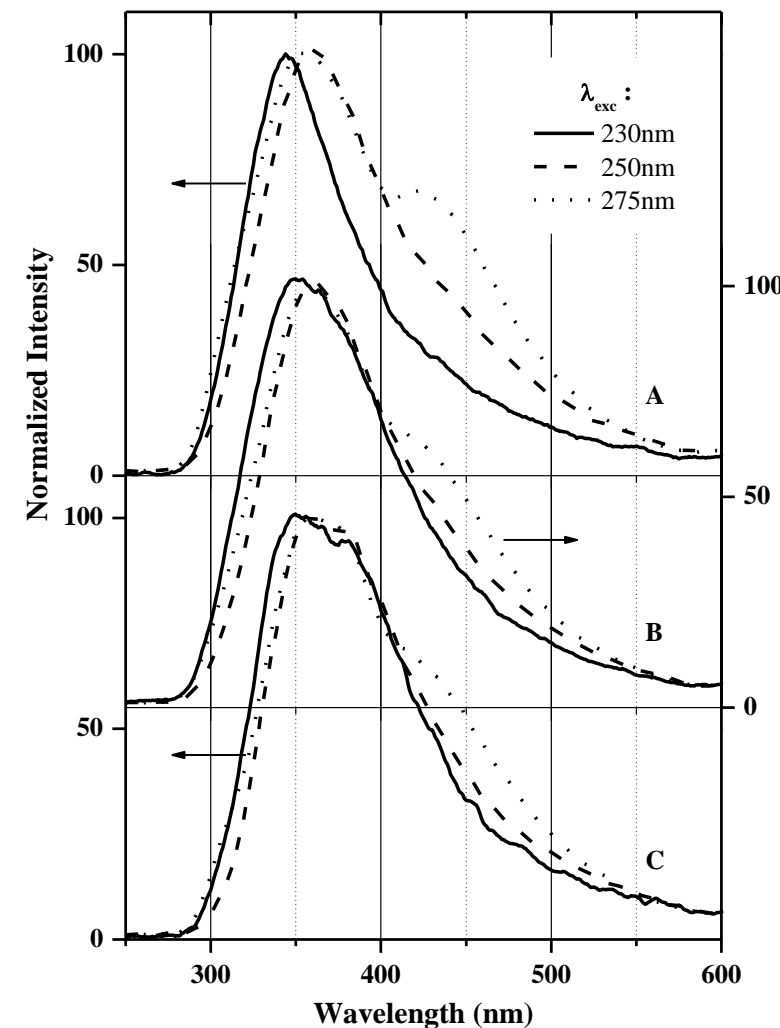

(a) Normalized emission spectra for different excitation wavelengths

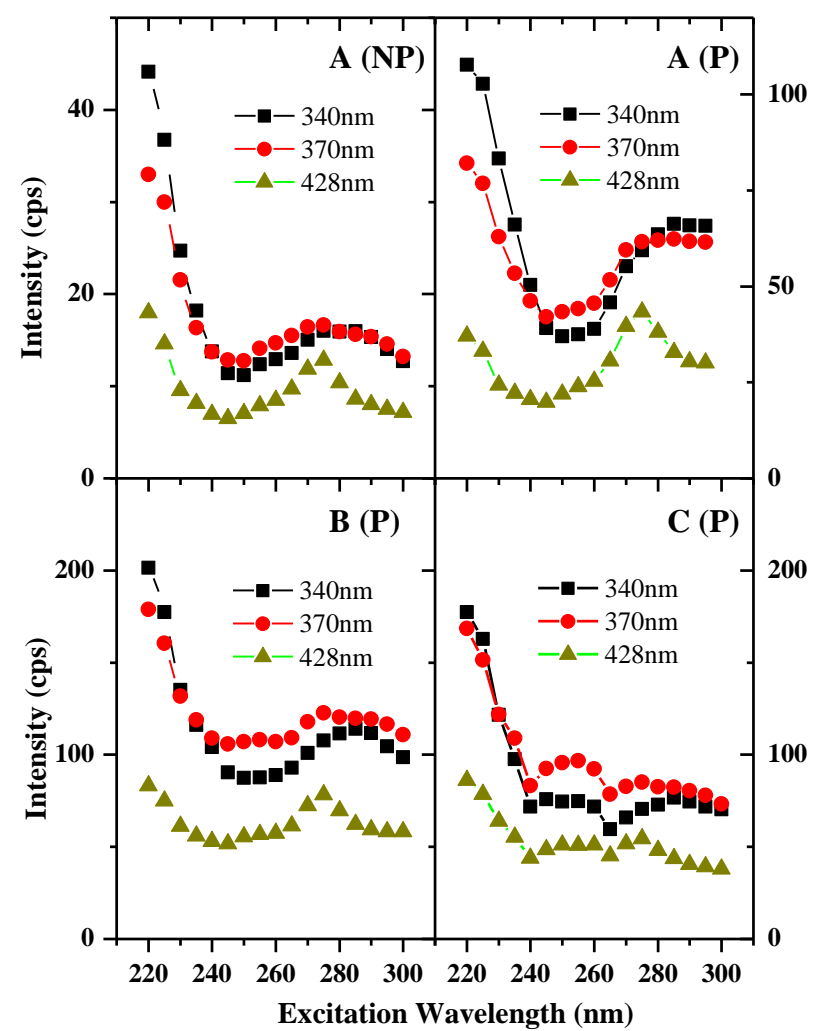

(b) Excitation spectra monitored at different emission wavelengths

Figure 15. Photoluminescence at $20^{\circ} \mathrm{C}$ from XLPE samples peeled from different cables $(A, B, C)$ from Artemis project. $\mathrm{P}=$ preconditioned (thermally treated); $\mathrm{NP}=$ not preconditioned. Adapted from [7]

-an emission at $340 \mathrm{~nm}$, which is best resolved when exciting at short wavelength (230 $\mathrm{nm}$, see Figure 15.a. The relative intensity of this band is weaker in samples B and C which contain less volatile species. Therefore, it might be characteristic of volatile groups. However, we did not detect any clear effect of preconditioning on the strength of the band. 
-an emission in the range 360-375 nm, with excitation band at about 280-290 $\mathrm{nm}$. Preconditioning strengthens this band. As emissions peaking at 340 and 360-375 nm are close-lying and relatively broad, the related excitation spectra cannot be easily distinguished. In sample C, a component at around $380 \mathrm{~nm}$ appears distinctly (see Figure 15.a). It is also detected as a shoulder for the other two cables, especially for the spectrum under excitation at $250 \mathrm{~nm}$. Considering the excitation spectra, this component corresponds to a peak at $250 \mathrm{~nm}$ in the excitation spectrum of sample $C$ which is not seen for the other samples. The growing of this band is possibly in relation to the fact that cable $\mathrm{C}$ was stored for a long time after cable production.

-an emission at around $425 \mathrm{~nm}$ with excitation peak at $275 \mathrm{~nm}$. The fluorescence of low density polyethylene is comparatively simpler (see Figure 13). Hence, this emission is clearly a characteristic of crosslinked polyethylenes. It seems not to be related to volatile species since preconditioning enhances it. In case of XLPE, the emission is strengthened by a factor of about 3 after preconditioning. We observed that it still increased in a sample previously treated at $50{ }^{\circ} \mathrm{C}$ for 2 days and kept at this temperature for one extra day. One would expect a decrease of the emission rather than an increase since volatile products, which are candidate as emitters, are removed at higher temperature. However, the increase of the emission does not necessarily mean that more emitting species are produced. It could result from removal of either strongly absorbing species which do not emit and/or from efficient quenching (energy transfer) of the excitation of the emitting groups by non-emitting species. In the first situation, one would expect a different UV-vis transmission spectrum after thermal treatment. In the second case, the change of shape of the excitation spectrum would be indicative of different quenching efficiency and/or difference in the volatility of emitting species. In any case fluorescence measurements can be indicative of the quantity of remaining by-products.

\section{c) Phosphorescence}

The emission spectra obtained at $-130{ }^{\circ} \mathrm{C}$ are shown in Figure 16. Results for as-received and preconditioned samples $\mathrm{A}$ are compared. These spectra are representative of what was obtained on the other materials. The excitation spectra (not shown) exhibit two maxima, at about 240-250 nm and $280 \mathrm{~nm}$ for all the samples. They differ markedly from the excitation spectrum of fluorescence, which means that phosphorescing species are different from fluorescing species. A strongly structured emission, significantly dependent on the excitation wavelength, was obtained for the as-received material. This structure is best resolved when exciting at $240 \mathrm{~nm}$. Several maxima are found at 388, 415, 443, 476, and $515 \mathrm{~nm}$.

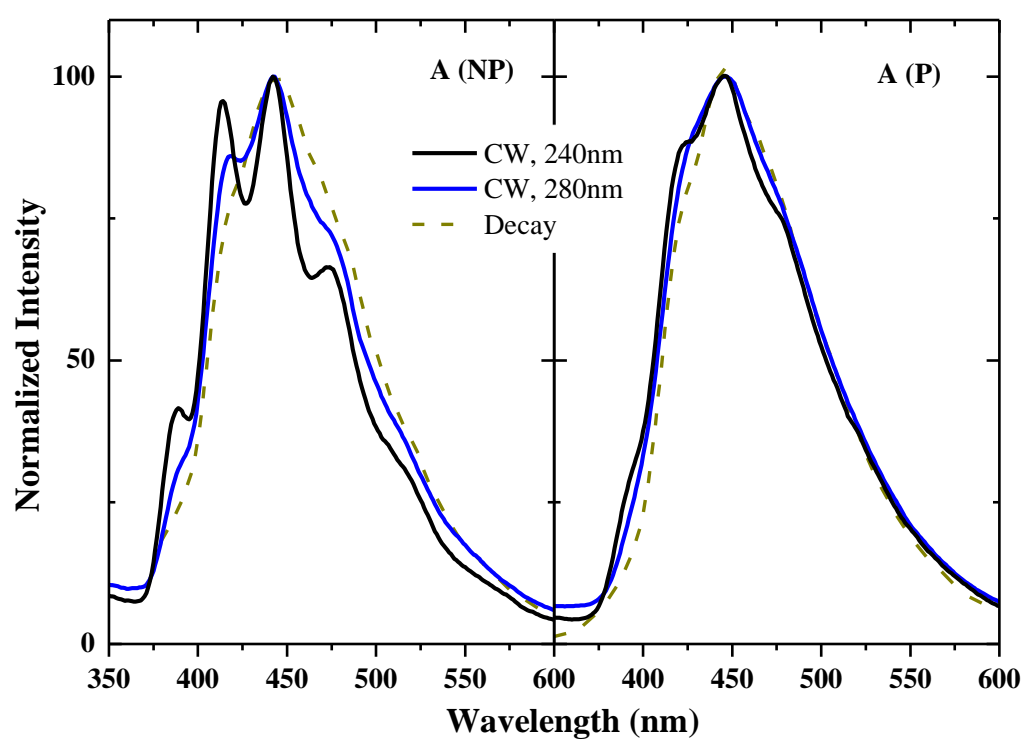

Figure 16. Phosphorescence spectra obtained at low temperature on as-received (NP) and preconditioned (P) sample A. The spectra during the decay were obtained with an integration time of $1 \mathrm{~s}$. CW=continuous wave excitation. Adapted from [7] 
These results show that at least two kinds of species contribute to the phosphorescence emission. They have distinct excitation spectra with maxima estimated at around $240 \mathrm{~nm}$ and $280 \mathrm{~nm}$. As for fluorescence (see Figure 15.b), preconditioning of sample $A$ increased the emission by a factor $\approx 2$. After preconditioning, the spectrum did not evolve greatly when changing the excitation wavelength. Upon excitation at $240 \mathrm{~nm}$, the structure described above was also detected. The spectrum obtained along the phosphorescence decay is also represented on Figure 16. Our present understanding of these phosphorescence spectra is that at least two kinds of species contribute to the emission: acetophenone and an unspecified one. There is no doubt that the structured emission is related to acetophenone. The position in wavelength of the bands is in good agreement with the literature data as will be seen later.

\section{d) RIL spectra}

Figure 17 compares the RIL spectra obtained for the different materials that have been tested. Except for preconditioned sample $A$, the spectra for the different materials are approximately the same. Structures can be distinguished at about 420,450, and $475 \mathrm{~nm}$. Though these spectra are broader than those presented in Figure 16, they exhibit the same components.

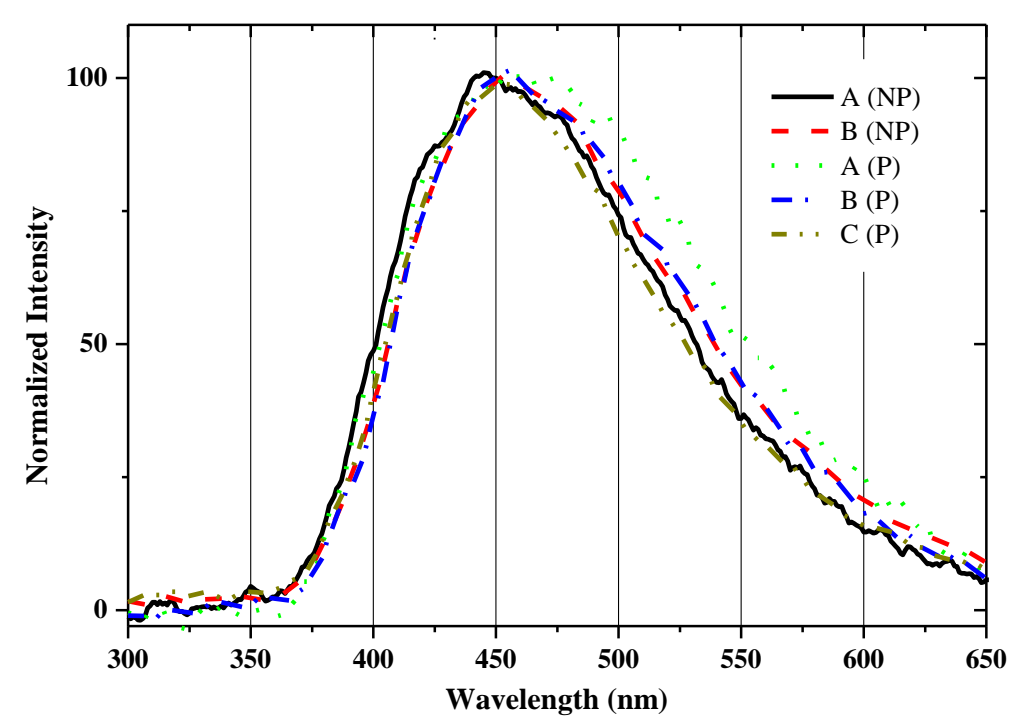

Figure 17. RIL spectra of the different materials tested. NP and P stand for not preconditioned and preconditioned, respectively. Adapted from [7].

\section{e) Luminescence spectra obtained on other kinds of XLPE samples}

The results presented above have been obtained on slices taken from the insulation of power cables. Here we report on results on thin films, either after a thermal treatment, or cross-linked from different base resins.

The signature at $428 \mathrm{~nm}$ in fluorescence emission is confirmed. Being not due to volatiles species, it should be in relation with strongly bonded species.

There is superposition of the UV phosphorescence and RIL spectra, as previously reported. The centers responsible for deep trapping in XLPE are not those identified in LDPE and they are strongly bonded to the hydrocarbon chain. 
Main features for luminescence in XLPE

- Main fluorescence peak at 350-370 nm and main phosphorescence peak at $450 \mathrm{~nm}$

- Confirmation of the component at $428 \mathrm{~nm}$ in fluorescence

- Confirmation of the superposition of UV phosphorescence and RIL spectra

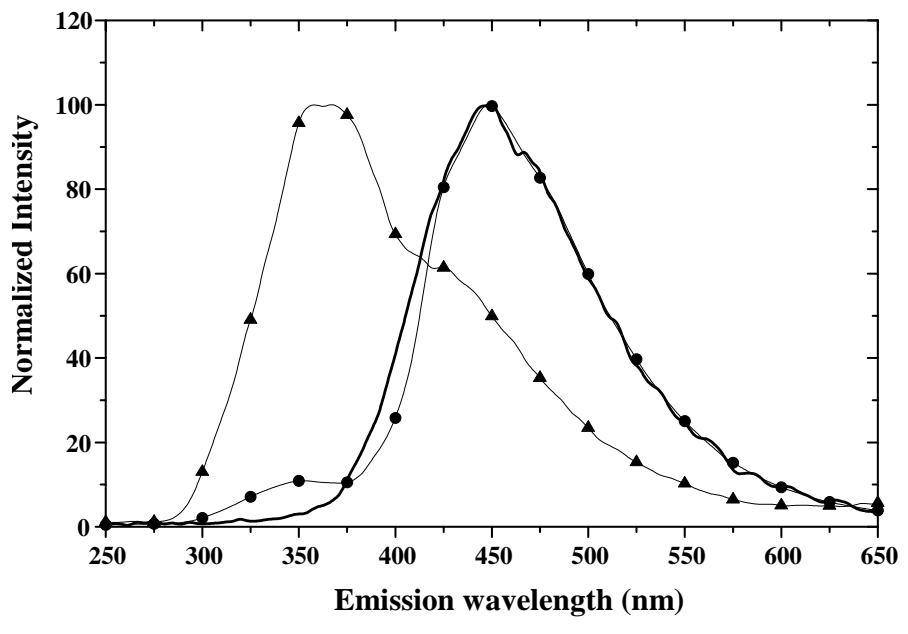

Figure 18. Luminescence spectra from XLPE under film form (by-products expelled). Photoluminescence: at RT; : at low temperature. $\lambda_{\text {exc }}=270 \mathrm{~nm}$. RIL spectrum (-) [54].

Main features for the impact of LDPE resin

- No visible impact of the nature of the LDPE resin (different melt flow rates) on the final response.

- Confirmation of the identical features for UV-phosphorescence and RIL

- Confirmation of the superposition of UV phosphorescence and RIL spectra

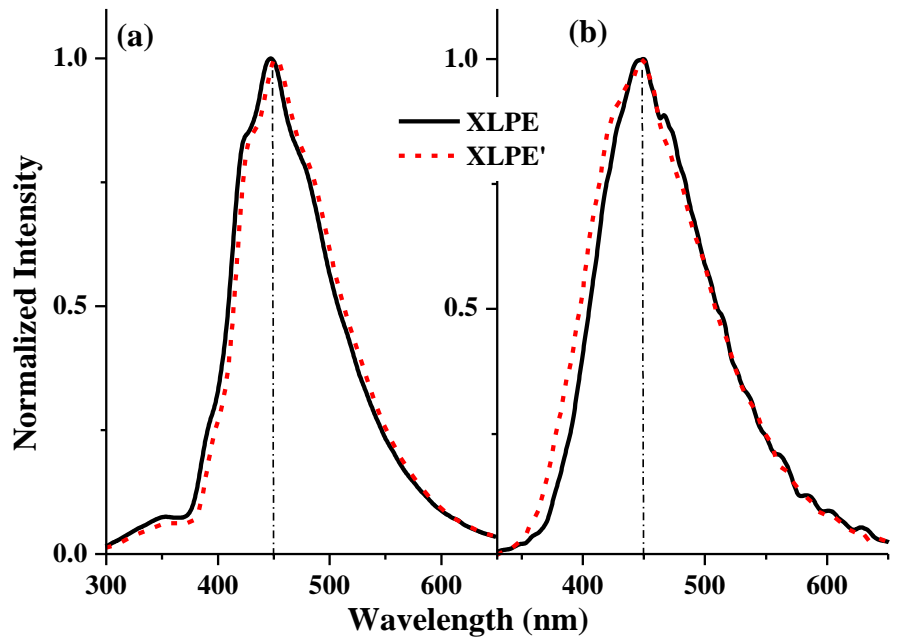

Figure 19. Luminescence features of XLPE (solid line) and XLPE' (dashed line) at low temperature $\left(-110^{\circ} \mathrm{C}\right)$. (a) normalized phosphorescence spectra for excitation at $250 \mathrm{~nm}$; (b) normalized RIL spectra. From [58].

\section{IV-1-3-Overall: LDPE vs. XLPE}

On the way of understanding XLPE intrinsic recombining centers, we have compared LDPE and XLPE photoluminescence/RIL spectra. If the origin of the LDPE luminescence properties is relatively well understood, this is contrary to the case of XLPE which exhibits specific properties. If the importance of by-products of the cross-linking reactions is well described as revealed by differences between spectra of treated and non treated films, the UV-photoluminescence and the RIL spectra on treated films are identical but specific to the XLPE. This demonstrates the recombining centers are strongly bonded to the hydrocarbon chains. Their origin cannot be linked with differences in the cross-linkable base resin as the spectra obtained on two XLPE with different base resins are identical. Identification of their origin needs model materials to be synthetized and tested. This is the object of the following paragraph. 


\section{IV-2-Model compounds for interpretation of XLPE deep trapping sites}

IV-2-1-Luminescence signature of model compounds: by-products of cross-linking reaction

Figure 20 shows a summary of PL and RIL data obtained with soaking LDPE with the three main by-products of crosslinking [54].

\section{a) LDPE plus acetophenone}

The luminescence spectra of LDPE samples soaked into acetophenone with about $1 \%$ as final concentration are presented in Figure 20.a. The fluorescence exhibits the same features as in LDPE with about the same emission yield. Depending on the excitation conditions, emission is found at $335 \mathrm{~nm}$ or in the range $360-380 \mathrm{~nm}$. As the only similarity between these two samples is the base resin, it is straightforward to conclude that the fluorescence is due to the base resin itself. The fact that acetophenone does not contribute to fluorescence is in accordance with the literature which points out that acetophenone is only a phosphorescent dye with no fluorescence [63]. The phosphorescence spectrum exhibits a structured emission with bands at 390, 418, 445 and $480 \mathrm{~nm}$ upon excitation between 240 and $250 \mathrm{~nm}$. The shape of the spectrum is the typical signature of acetophenone emission [64] [51], along with the short phosphorescence lifetime which does not exceed $10 \mathrm{~ms}$. This structure arises from relaxation of the triplet states involving ground state vibrations of the chromophore and the difference in energy between the bands is related to the $v(C=O)$ vibration band. The recombination spectrum does not fit the phosphorescence spectrum and, in particular, it does not display the typical signature of acetophenone within LDPE. However, the RIL spectrum is stretched towards the short wavelengths relative to that of LDPE. The strengthening of the emission in the region 380-450 $\mathrm{nm}$ probably arises from a contribution of the acetophenone. Then, acetophenone probably acts as a recombination centre, in addition of the LDPE recombination centers.

\section{b) LDPE plus cumylalcohol}

The fluorescence spectrum of the cumylalcohol-containing sample (Figure 20.b) presents two peaks at $318 \mathrm{~nm}$ and $329 \mathrm{~nm}$ correlated with two maxima in the excitation spectra at $225 \mathrm{~nm}$ and $255 \mathrm{~nm}$ respectively. These features are unique in the set of materials studied. The fluorescence component is most probably due to the cumylalcohol itself and the fluorescence of the base resin is overwhelmed.

The phosphorescence spectrum clearly shows a structure with peaks at 438, 465 and $491 \mathrm{~nm}$ when the excitation wavelength is at about $260-270 \mathrm{~nm}$. It is noteworthy that the excitation spectrum is the same as for fluorescence. This structure is therefore assigned to the phosphorescence of cumylalcohol. The RIL spectrum clearly fits with the cumylalcohol phosphorescence spectrum though the structures are smoother. Therefore, cumylalcohol acts as an efficient recombination centre.

\section{c) LDPE plus alpha-methylstyrene}

The fluorescence spectra of $1 \%$ containing alpha-methylstyrene LDPE looks like cumylalcohol containing LDPE. There are two maxima at $317 \mathrm{~nm}$ and $325 \mathrm{~nm}$, correlated with maxima in the excitation spectra at $220 \mathrm{~nm}$ and 250-255 $\mathrm{nm}$ respectively. The possible slight difference in emission/excitation wavelengths at peak maximum relatively to cumylalcohol could be due to the presence of $\mathrm{OH}$ group in the latter that shifts the emission peak towards longer wavelength [6]. Phosphorescence is weak if any. The free rotation of the phenyl group around $\mathrm{C}-\mathrm{C}$ bond (which is constrained in the case of cumylalcohol due to the electronegative oxygen atom) could be accounted for. 
(a) LDPE + Acetophenone

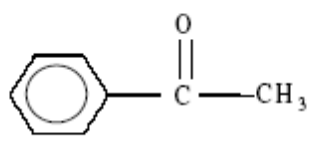

- UV-fluorescence consistent with LDPE base resin considering excitation wavelength dependence and in agreement with non fluorescent character of acetophenone

- UV-phosphorescence assigned to acetophenone with vibronic bands typical of $\mathrm{C}=\mathrm{O}$ groups.

- RIL shifted to the blue/LDPE. Acetophenone contributes but LDPE contributing species at longer wavelengths.

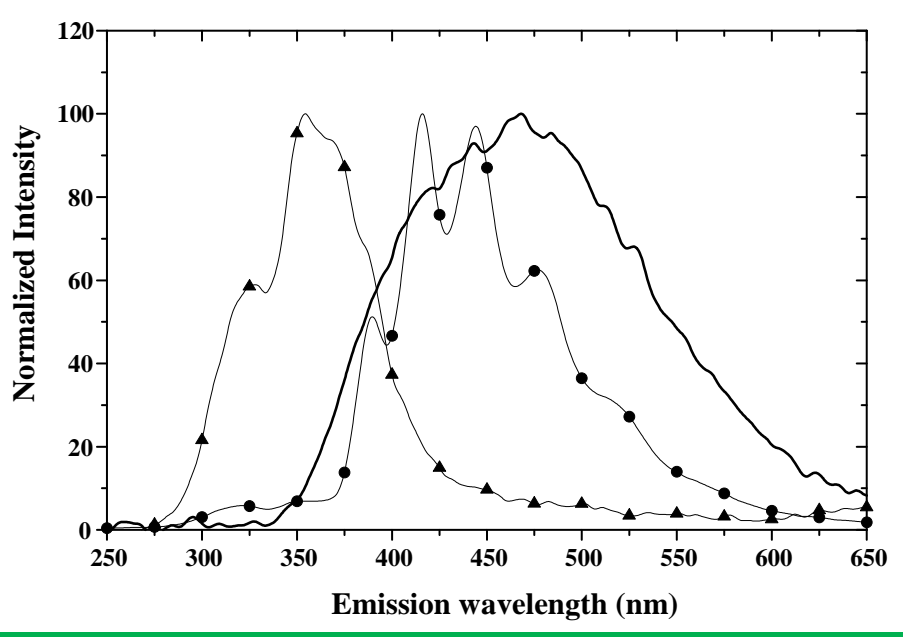

(b) LDPE + Cumylalcohol<smiles>CC(C)(O)c1ccccc1</smiles>

- UV-fluorescence peak at $329 \mathrm{~nm}$ with a weak dependence on excitation wavelength: assigned to cumylalcohol fluorescence

- UV-fluo and phospho have the same excitation spectra

- UV-phosphorescence and RIL identical with typical signature of cumylalcohol

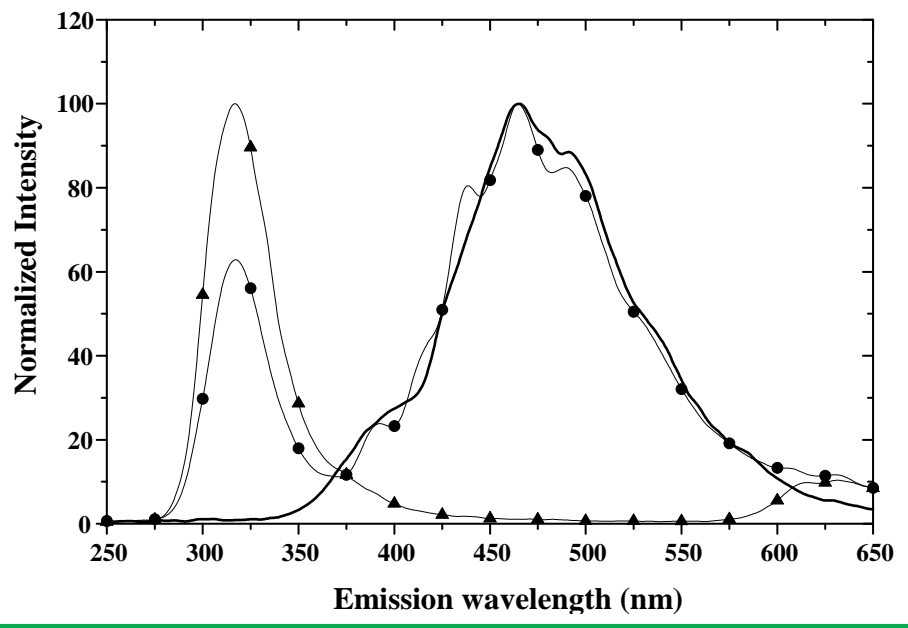

(c) LDPE $+\alpha$-methylstyrene<smiles>C=C(C)c1ccccc1</smiles>

- UV-fluorescence is almost that of cumylalcohol containing LDPE

- UV-phosphorescence is weak if any / fluorescence (possibly traces of acetophenone)

- Broad RIL spectrum like for acetophenone

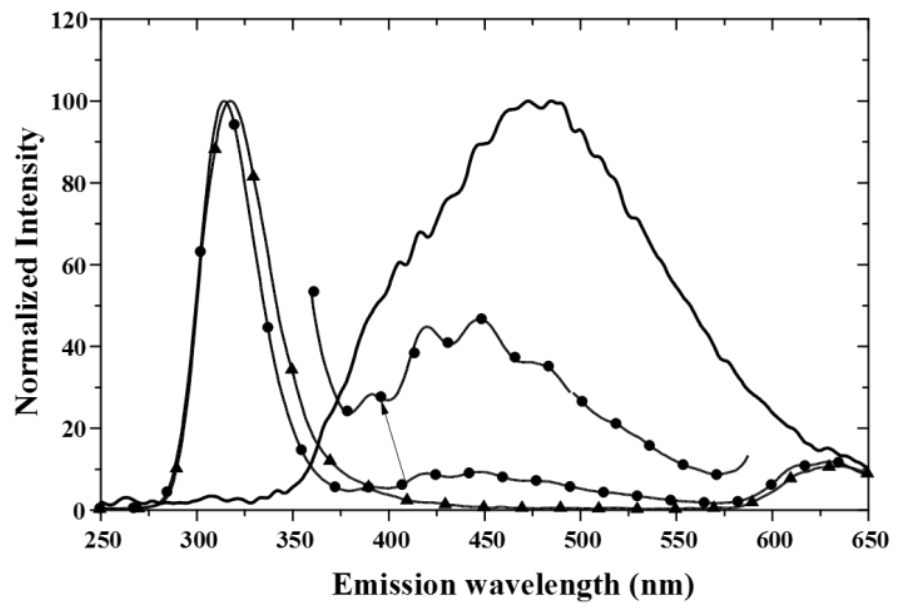

Figure 20. Luminescence spectra from LDPE samples dipped at $\approx 1 \% \mathrm{w}$./w. into by-products.

(a) acetophenone, PL: $\lambda_{\text {exc }}=250 \mathrm{~nm}$; (b) cumylalcohol, $\lambda_{\text {exc }}=270 \mathrm{~nm}$; and (c) $\alpha$-methylstyrene $\lambda_{\text {exc }}=240 \mathrm{~nm}$.

Photoluminescence: $(\boldsymbol{\Delta})$ at RT; $(\bullet)$ : at low temperature. RIL spectrum (一) [54]. 
IV-2-2-Luminescence signature of XLPE model compounds with grafted species

We consider here two kinds of grafted species onto XLPE. Both are aromatic groups. The difference with the previous case is that those molecules can't diffuse and be released from the material after processing. The corresponding PL and RIL spectra are shown in Figure 21.

(a) XLPE-g-Benzophenone<smiles>[R]CCCC(C)CC(=O)Oc1ccc(C(=O)c2ccccc2)cc1</smiles>

- UV-fluorescence is weak

- UV-phosphorescence has the same excitation spectrum as fluorescence

- UV-phosphorescence and RIL characteristic of grafted benzophenone
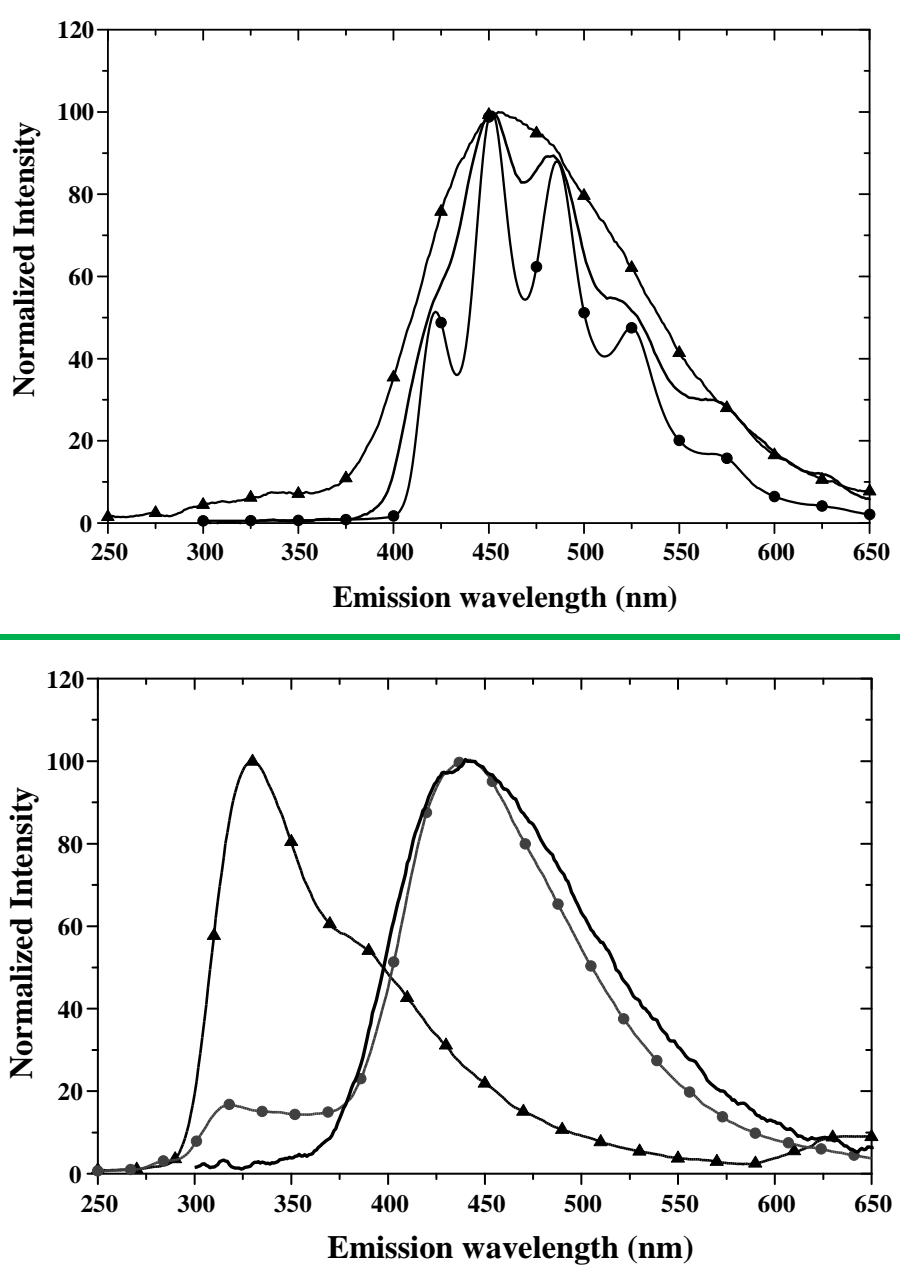

Figure 21. Luminescence spectra from XLPE with grafted molecules.

(a) benzophenone derivative, PL: $\lambda_{\text {exc }}=270 \mathrm{~nm}$; (b) styrene, $\lambda_{\text {exc }}=280 \mathrm{~nm}$. Photoluminescence: ( $\mathbf{\Delta}$ ) at RT; (•): at low temperature. RIL spectrum (-) Adapted from [10].

\section{a) XLPE plus grafted benzophenone}

The photoluminescence spectrum at room temperature is constituted of a weak fluorescence peaking at about $340 \mathrm{~nm}$ and a broad band with maximum at $450 \mathrm{~nm}$ and some structures at both sides as shown in Figure 21.a. By comparison to the spectrum obtained at $80 \mathrm{~K}$, it is clear that this broad band corresponds to phosphorescence of the material. The emission in this wavelength range is increased by a factor of about 15 upon cooling. A strongly structured emission with bands at 422, 451, 487, 526, 570 and $624 \mathrm{~nm}$ appears in the low temperature spectrum. This structure is consistent with the one reported for the phosphorescence of benzophenone [63]. The only difference is a red-shift by about $7 \mathrm{~nm}$ of the phosphorescence with respect to that of benzophenone, which is attributable to the fact that a benzophenone derivative is grafted on to the XLPE matrix. It is worth noting that the spectrum does not evolve to a great extent when the excitation wavelength is changed and that the phosphorescence lifetime is very short (some ms). The RIL spectrum matches very well with the phosphorescence structure the only difference being some broadening of the 
bands. The recombination process is assumed to be entirely controlled by the species with a characteristic spectrum identified as the phosphorescence spectrum. From the integral light decay signal, it was observed that the radiative charge recombination contribution dominates the decay of the PIL in a large time range $(t>8$ s). The grafted species therefore acts as a very efficient trapping centre.

\section{b) XLPE plus grafted styrene}

We carried out PL measurements on a $1 \%$ grafted styrene and $5 \%$ grafted styrene XLPE (same spectral features for a given excitation wavelength, same excitation maxima). The only important difference is in the photoluminescence yield which is about twice for the sample with $1 \%$ styrene, that is to say the luminescence is not a linear function of the phenyl chromophore concentration. This behavior is well known and can be due to non radiative de-excitation routes (intramolecular quenching) which are open when nearby chromophores are able to interact. This is likely to happen when the grafted styrene concentration is increased from $1 \%$ to $5 \%$. As shown in Figure 21.b, the emission peaking at $325 \mathrm{~nm}$ (RT) has an excitation maximum between 250 and $260 \mathrm{~nm}$ which fits the absorption peak of benzene ring in hexane solution, leading us to assign it to the fluorescence of the $\pi$ orbital system of the phenyl group [64] [65]. This is further supported by considering the fluorescence emission of polystyrene that is reported between $310 \mathrm{~nm}$ and $330 \mathrm{~nm}$, depending on the commercial source and purity of the polymer [6]. It is tempting to associate the $440 \mathrm{~nm}$ emission observed at LT to the phosphorescence emission of the phenyl ring. However, phosphorescence has not the same excitation spectrum as fluorescence, which indicates that the two emissions could have a different origin. Polystyrene phosphorescence has been reported in the same energy domain and assigned to acetophenonetype terminal groups [6]. On another hand, the phosphorescence spectrum of Figure 20.b is not strongly structured contrary to what is observed in the case of acetophenone-type emission, and the general shape is not the same. We therefore attributed the phosphorescence peak to the presence of the phenyl groups. XLPEsty (with $5 \%$ or $1 \%$ grafting) exhibits also a fluorescence emission at wavelengths 335,355 , and $390 \mathrm{~nm}$ on excitation with light of wavelengths larger than $300 \mathrm{~nm}$. This emission has already been reported as an "anomalous" fluorescence in polystyrene [66], and has been assigned to the presence of trans-stilbene linkages of the form given below:

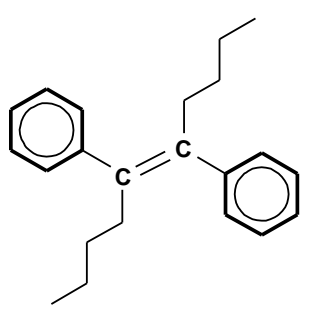

Confirmation of the involvement of phenyl groups in fluorescence will be given in the next section.

IV-2-3-Luminescence signature of model compounds with anti-oxidant and its derivatives

\section{a) LDPE base resin plus anti-oxidant}

The samples used refer to LDPE containing $0.2 \%$ of an antioxidant (Santonox). The PL and RIL spectra are shown in Figure 22.a Relative to LDPE, the fluorescence spectra have in common the band at $335 \mathrm{~nm}$. This band is strengthened for short excitation wavelengths for both materials. However, when a longer excitation wavelength is used (in the range 250-260 nm), another emission is found at $354 \mathrm{~nm}$, being lower in magnitude than the emission at $360 \mathrm{~nm}$ found in LDPE. The fluorescence intensity is much weaker, and therefore it seems that the antioxidant by itself does not add a significant extra-fluorescence contribution. The differences are attributed to the protecting action of the antioxidant. Considering that the long wavelength part of the fluorescence spectrum is related to oxidation, the shape of the spectrum may be explained by prevention of oxidation by the antioxidant. 
The phosphorescence spectrum is relatively broad and structureless as observed in LDPE. However, it is significantly blue-shifted, compared to LDPE, with a maximum peaking at $444 \mathrm{~nm}$. The long-wavelength part of this phosphorescence spectrum is probably broadened by the response of the species emitting in LDPE. So, it can be inferred that the antioxidant phosphoresces with a maximum of emission at around $440 \mathrm{~nm}$ which rather favourably compares to the $430 \mathrm{~nm}$ reported in the literature [6], bearing in mind that the chemical composition of such a product is really complex. The RIL spectrum is again clearly in the phosphorescence domain. It is also markedly different from the one obtained in LDPE. As this RIL spectrum fits well to the phosphorescence spectrum, it can be concluded that the antioxidant and/or its byproducts are an efficient recombination centre.

\section{b) LDPE base resin plus reaction products of the antioxidant}

Another important potential source of chemical traps is provided by the reaction products of the antioxidant, i.e. molecular groups created by reaction of $\mathrm{AO}$ with oxidative agents such as peroxide and oxygen, thus preventing polyethylene oxidation, mainly during the crosslinking process. The reaction products of the antioxidant (4, 4'-thiobis (2-tertbutyl-5-methylphenol), AO-) can be classified in three categories labelled as AOR1 and AOR2, corresponding respectively to 1 and 2 oxidation levels of the sulfide function, and AOR3 consisting of an oligomer form of the phenolic function, the respective chemical formula being given in section III-1-2 and recalled in Figure 22. They were incorporated into LDPE base resin at a level of $0.1 \%$ per weight, and then films of typically $130 \mu \mathrm{m}$ thickness were produced by press-moulding at $130^{\circ} \mathrm{C}$ (thermoplastic materials) or $180^{\circ} \mathrm{C}$ (crosslinkable materials).

RIL and photoluminescence spectra of LDPE-AO and related reaction products are clearly different from the one of LDPE. This means that the emissions in the model materials are characteristic of these additives. The structure found in the RIL spectrum of LDPE AO is clearly revealed in two of the model compounds of AO reaction, LDPE-AOR1 and LDPE-AOR2. Both exhibit emission bands peaked at 390, 415, 445, 478 and $510 \mathrm{~nm}$. Such structured RIL spectra have been observed so far only in one instance, with a benzophenone derivative grafted on the LDPE chain (see above). Occurrence of a structured emission in phosphorescence spectra corresponds to relaxation of triplet states involving ground state vibrations of the chromophore. An example of this is luminescence from centres involving carbonyl groups, giving bands equally spaced in energy by the $v$ $(C=0)$ vibration energy and enhanced structuration when $\left(n, \pi^{*}\right)$ states are involved [51] [67]. Out of the four $\mathrm{AO}$ and $\mathrm{AO}$ derivatives containing samples, the two materials exhibiting the strongest structure in the RIL spectrum are those being oxidized to 1 or 2 degrees, that are those containing $\mathrm{S}=\mathrm{O}$ or $\mathrm{O}=\mathrm{S}=\mathrm{O}$ groups. It can then be speculated that the structure is related to these groups. The structure is however less clear when considering photoluminescence spectra. This might be because both $\left(n, \pi^{*}\right)$ and $\left(\pi, \pi^{*}\right)$ states coexist, due to the oxygen doublet, and that charge recombinations involve preferably $\left(n, \pi^{*}\right)$ states, giving rise to a structure, whereas photoluminescence involves preferably $\left(\pi, \pi^{*}\right)$ states, giving rise to more diffuse spectra.

The important output of this study is that the typical UV-phosphorescence and RIL spectra of XLPE have been reproduced in materials containing AO reactions products (AOR1 and AOR2). Spectra of LDPE-AO exhibit a weaker structure whereas the phosphorescence emission of the antioxidant itself is normally structure-less and has peak at $430 \mathrm{~nm}$ [6]. It is however likely that part of the AO has reacted during film processing and this could be the reason for observing discernable structures. The explanation is supported by the fact that the photoluminescence yield in AOR1 and AOR2 is much stronger than for the other materials and thus AO reaction products can be detected, even if in low quantity. Finally, AOR3, the oligomer form of the AO, exhibits structure-less photoluminescence and RIL spectra with comparable features with LDPE. AOR3 does not seem to participate to the response of XLPE, contrary to AO, AOR1 and AOR2. 
(a) LDPE + Antioxidant

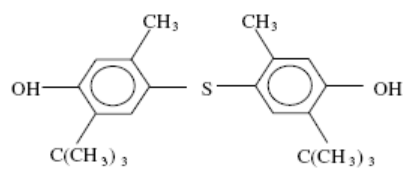

- UV-fluorescence blue-shifted /LDPE: assigned to protecting effect of antioxidant

- UV-phosphorescence blue-shifted/LDPE: assigned to a contribution of the antioxidant itself or to its reaction products

- UV-phosphorescence identical to RIL spectrum : assigned to the antioxidant itself or to its reaction products

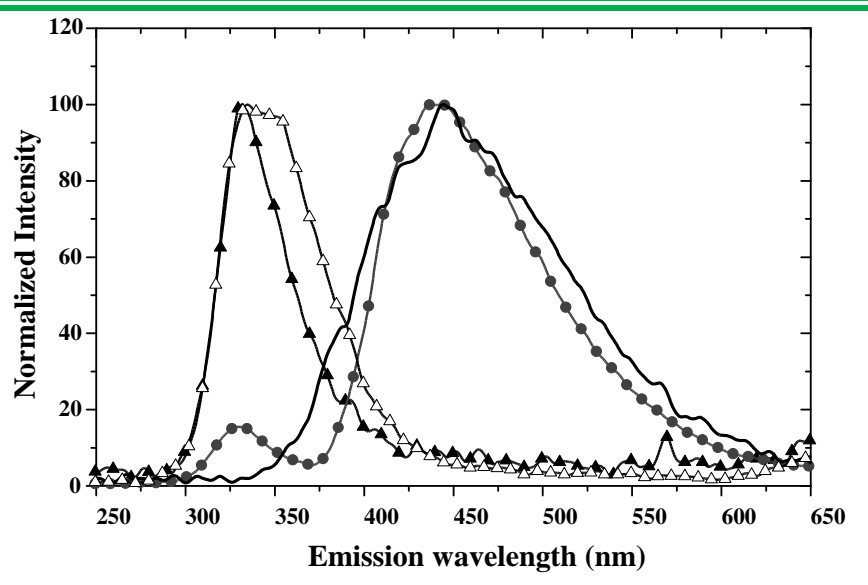

(b) LDPE + AO Reaction Product 1

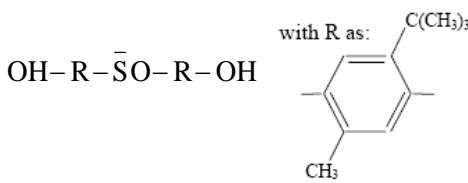

- Fluorescence has the characteristics of LDPE base resin fluorescence

- Identical spectra of UV phosphorescence and RIL, the later with structures at $390 \mathrm{~nm}, 414$ $\mathrm{nm}, 444 \mathrm{~nm}, 479 \mathrm{~nm}$

- Comparable to the spectra of AO containing LDPE

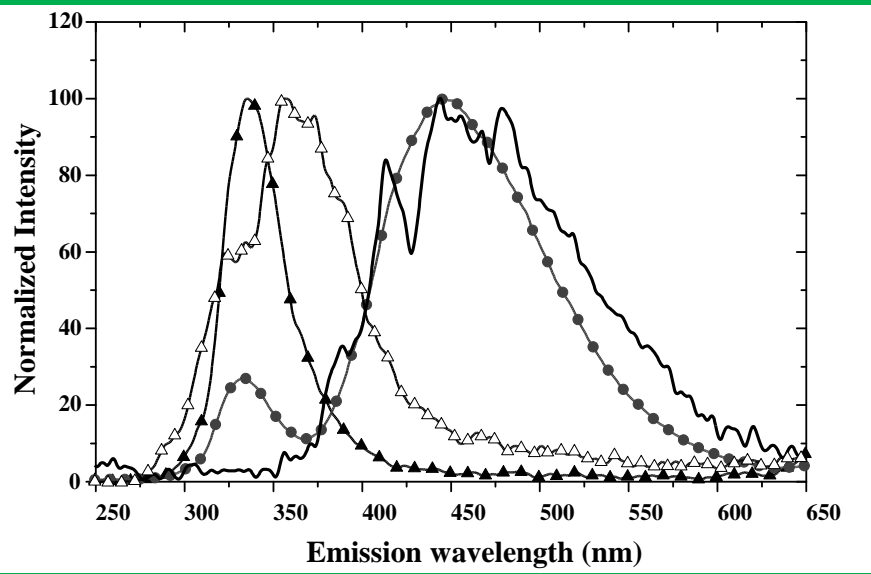

(c) LDPE + AO Reaction Product 2

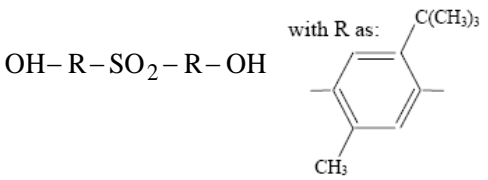

- Fluorescence has the characteristics of LDPE base resin fluo

- Identical spectra of UV phospho and RIL, the later with structures at $389 \mathrm{~nm}, 415 \mathrm{~nm}, 444$ $\mathrm{nm}, 480 \mathrm{~nm}$

- Comparable to the spectra of AO containing LDPE

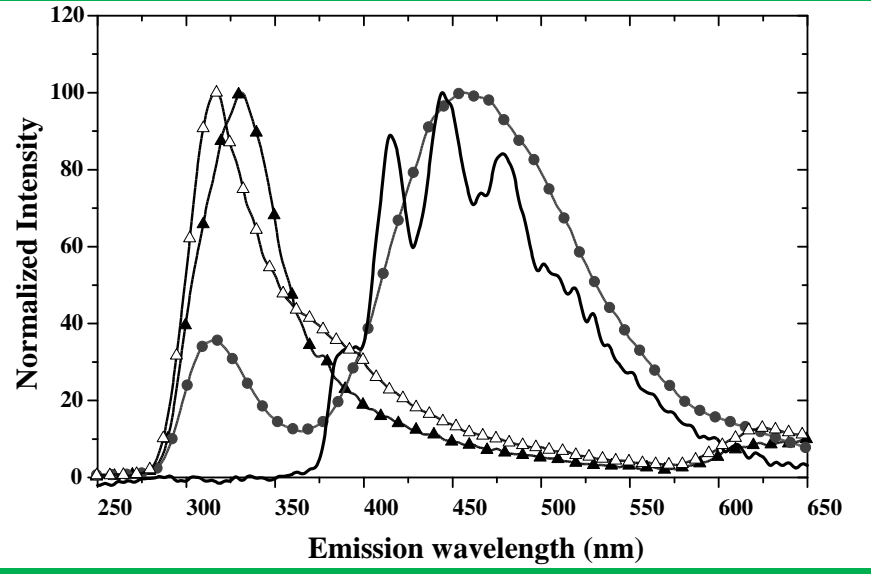

(d) LDPE + AO Reaction Product 3

$$
[-\mathrm{R}-\overline{\mathrm{S}}-\mathrm{R}-\mathrm{O}-]_{\mathrm{n}}
$$

- Fluorescence and RIL have the characteristics of LDPE base resin

- AOR3 does not seem to contribute to the luminescence signature

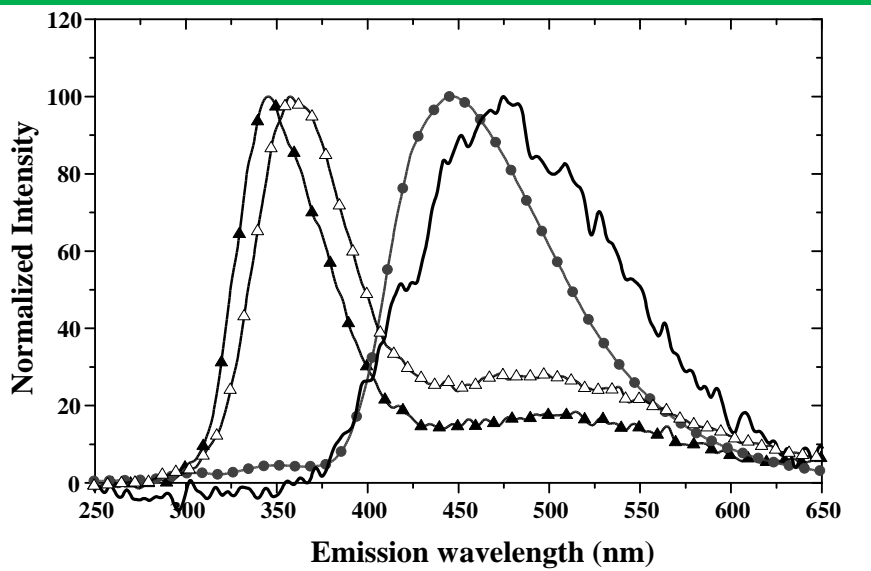

Figure 22. Luminescence spectra of LDPE with antioxidant and its reaction products.

PL: $(\boldsymbol{\Delta}) \lambda_{\text {exc }}=230 \mathrm{~nm} ;(\Delta) \lambda_{\text {exc }}=255 \mathrm{~nm}$ at RT; $(\bullet): \lambda_{\text {exc }}=225 \mathrm{~nm}$ at low temperature. RIL spectrum (一). 


\section{IV-3-Investigating cable XLPE insulation with screen diffusing species}

As example of the application of photoluminescence technique for investigating XLPE properties, we discussed here below the differences that exist when dealing with XLPE or XLPE coextruded with its semi-conductor screens as they exist in power cables. In the latter case, diffusing species from the semi-conductor screens are detected depending on the ageing conditions of the cable.

Samples were peeled from two $90 \mathrm{kV}$ HVAC cables produced by the cable manufacturers within the ARTEMIS project [68]. The major difference between the cables is the design of the cross-linking manufacturing systems at the two cable producers. Both the cable construction and the polymeric materials used are the same. Cables will be referenced to as $A$ and $B$, depending on the manufacturer. The cables have an insulation thickness of 14 $\mathrm{mm}$. The insulating material is a XLPE while the semi-conducting (SC) material (1.2 to $1.5 \mathrm{~mm}$ in thickness) is a carbon-black doped ethylene butyl acrylate copolymer [69]. Both materials contain antioxidants for thermal stabilization and peroxide for cross-linking. The cross-linking is accomplished by heating the cable core materials up to at least $180^{\circ} \mathrm{C}$ in a pressurized tube. Long sections of cables were aged in dry conditions for time up to $10000 \mathrm{~h}$, voltages up to $325 \mathrm{kV}$ (corresponding to a field of $31.2 \mathrm{kV} / \mathrm{mm}$ at the inner semiconducting layer) and temperatures of 20 or $90{ }^{\circ} \mathrm{C}$. The complete list of ageing conditions, 23 in total, is given in [68]. Peelings were cut from the cables using a lathe equipped with a specially designed knife to get optimum surface smoothness. They have a nominal width of $8 \mathrm{~mm}$ and a thickness of $150 \mu \mathrm{m}$. One full roll for each ageing condition was tested by PL. The length of insulation within each roll was approximately $14.5 \mathrm{~m}$. Except when otherwise stated, no thermal preconditioning was applied to the samples prior to the PL measurements reported here. Photoluminescence measurements were carried out at 21 positions along the roll length, the measurement points being tightened next to the inner and outer semi-conducting screens.

\section{IV-3-1-Influence of storage duration of XLPE slices in the presence of SC}

The first section of the cables was taken just after cable production. It was processed into a roll, the two ends of it being made of semi-conducting material (about $1 \mathrm{~m}$ long each). A section of that roll, corresponding to the region 2 to $3 \mathrm{~mm}$ from the inner SC of the cable was cut for the purpose of carrying out PL and other measurements, Figure 23. The two parts were then stored separately in plastic bags in lab-room conditions.

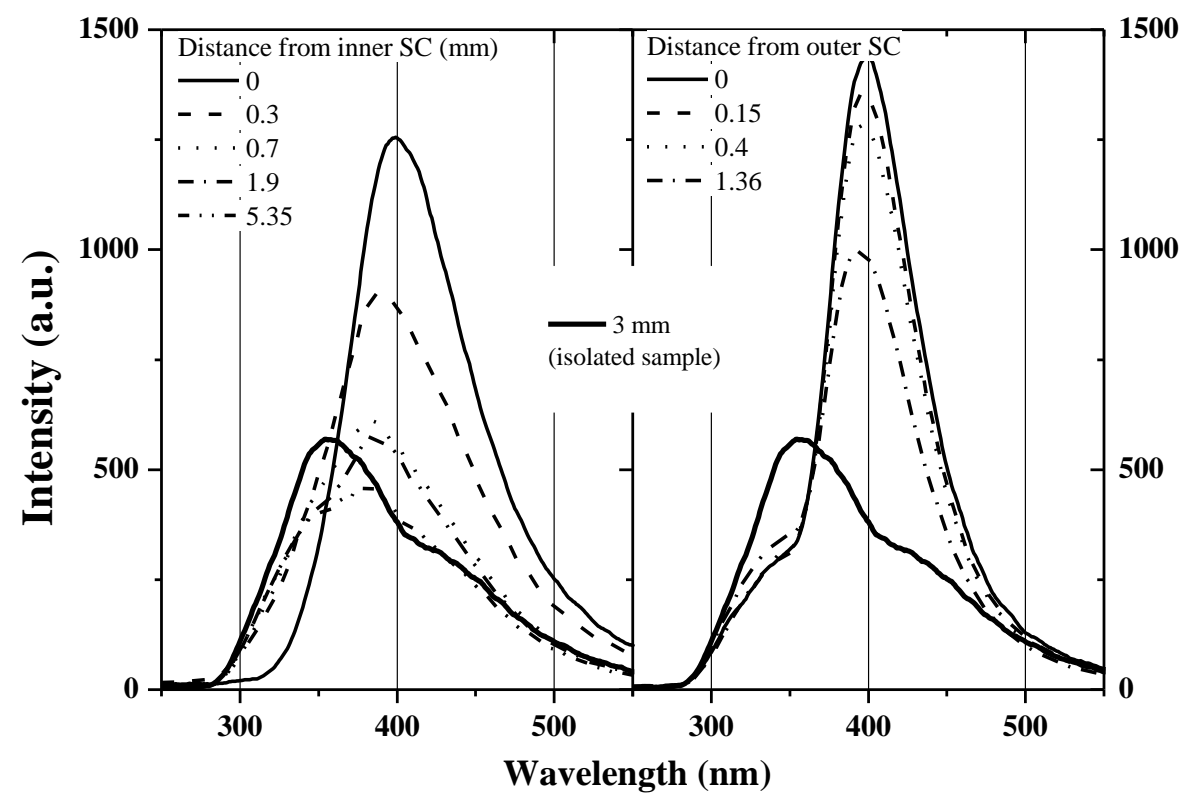

Figure 23. Bold line: PL spectrum obtained short after cable A production; sample taken at $3 \mathrm{~mm}$ from the inner screen. Thin lines: spectra obtained after 1 year of storage of the roll with the semi-conducting screen; sample position as indicated in the caption. Excitation wavelength $=275 \mathrm{~nm}$. From [56]. 
Figure 23 compares the PL spectra obtained short after roll production on the isolated sample taken at $3 \mathrm{~mm}$ from the inner SC to those obtained 1 year after storage of the roll, at various positions along the roll. Here and in the following results, the position ' 0 ' corresponds to XLPE at the edge of the SC. Clear differences are observed between these spectra: a band is detected with peak at about $400 \mathrm{~nm}$ except for the isolated sample which exhibits the previously reported characteristics of XLPE. The intensity of the band is all the stronger that the sample is taken close to either the inner or the outer SC. Initially, it was thought that diffusion of volatiles from the screen to the insulation had occurred during the 1 year roll storage. Hence, from then on, a minimum amount of SC was let attached to the ends of the rolls being produced and all PL measurements have been carried out short after roll distribution.

\section{IV-3-2-Fluorescence and phosphorescence vs. cable radius in unaged and aged samples}

Figure 24 shows the variation of the shape of the spectra along the cable radius along with the intensity monitored at two characteristic positions (335 and $400 \mathrm{~nm}$ ). A new roll of reference (i.e. un-aged) cable A was used for that purpose. We observe that, despite cautions taken between roll production and characterization, the emission at $400 \mathrm{~nm}$ is detected within almost all the bulk of the insulation. In Figure 24.a, it is clearly seen that the ratio $1400 \mathrm{~nm} / 1335 \mathrm{~nm}$ is the stronger close to the SCs. Also, the profile is clearly sharper close to the inner SC: this is a general feature of the results obtained in these measurements. For spectra taken in the range 1 to $6 \mathrm{~mm}$, the emission is dominated by features usually detected in XLPE (bands at $425 \mathrm{~nm}$ and in the range 350-375 nm, see Figure 23). After thermoelectric ageing (Figure 24.b), the emission at $400 \mathrm{~nm}$ dominates at any position in the dielectric. The profile for the emission at $400 \mathrm{~nm}$ becomes more flat, and again this is a general feature observed in these experiments.

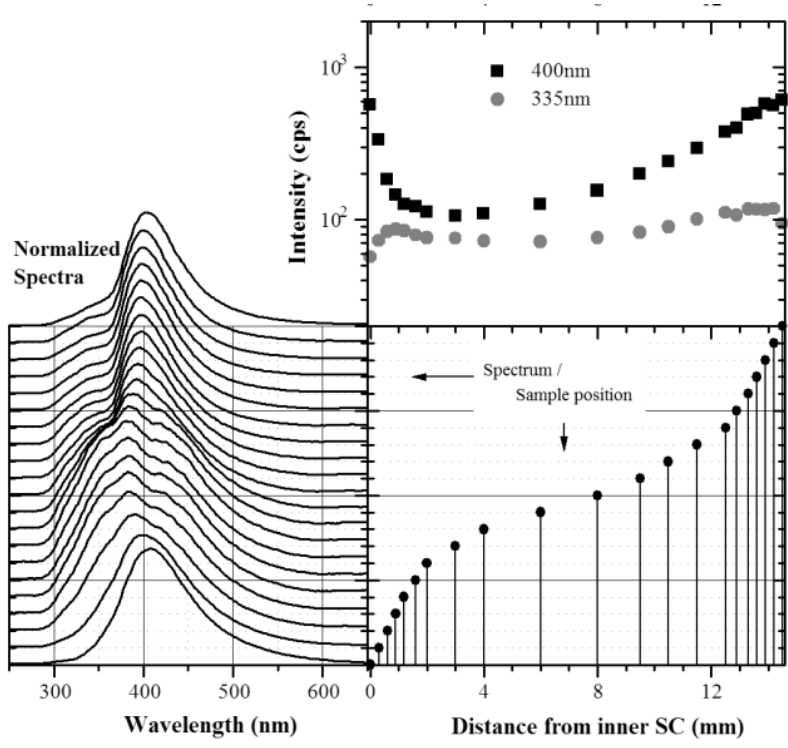

(a) Reference (unaged) cable A

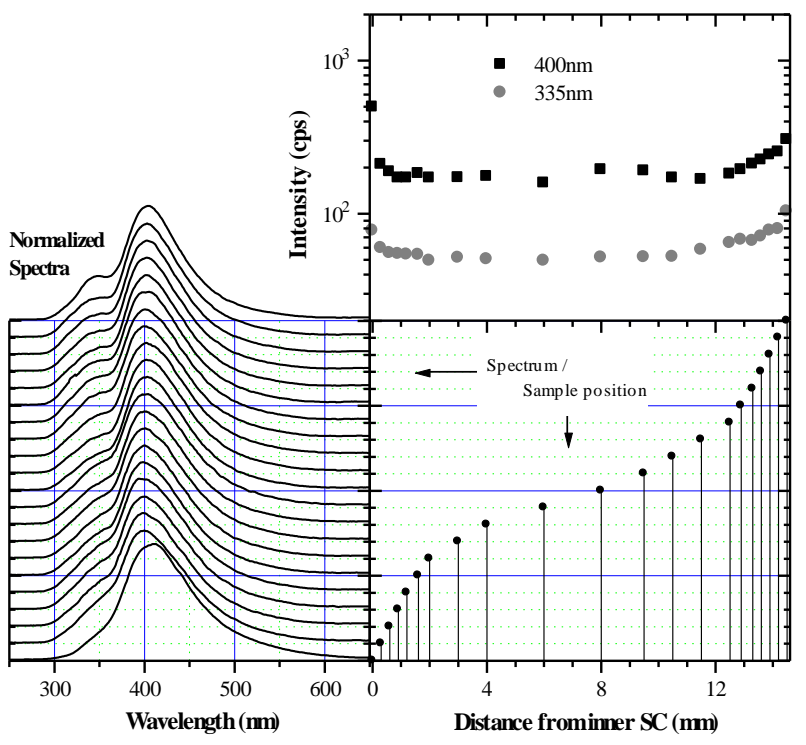

(b) Cable A aged for $7600 \mathrm{~h}$ at $90^{\circ} \mathrm{C}$ under $225 \mathrm{kV}$

Figure 24. Spectra obtained along the radius of the insulation of reference and aged cable A. Excitation wavelength $=275 \mathrm{~nm}$. Spectra were normalized to the intensity at emission maximum. The upper-right figure shows the intensity (given in counts per second) monitored at 335 and $400 \mathrm{~nm}$. The position of spectra along the roll is given in the lower-right figure. From [56].

A further proof of specific PL signatures close to the SC is given by photoluminescence measurements realized at low temperature, which bring information on phosphorescence processes. Figure $\mathbf{2 5}$ shows the spectrum obtained close to the inner SC, under continuous wave (CW) excitation at $250 \mathrm{~nm}$, and the spectrum obtained just after excitation shutter switch-off (decay spectrum). They are compared to the spectrum obtained on the isolated sample (see Figure 23) for the same cable roll at $3 \mathrm{~mm}$ from the inner SC. The emission in the bulk is dominated by the response of acetophenone with bands at 388, 415, 443, 476 and $510 \mathrm{~nm}$. We can see that spectrum (1), obtained under CW excitation, is a convolution of spectra (2) and (3). Consistently with what has 
been previously shown, the phosphorescence lifetime of acetophenone is extremely short (order of some ms), and its contribution is not detected in the decay spectrum (2). Several decay spectra were recorded consecutively in the time scale of several seconds, and no evolution was detected. This decay spectrum, which differs markedly from that obtained in bulk XLPE, exhibits clearly four bands at 461, 492, 522, and $567 \mathrm{~nm}$. It is supposed that the same specie as that which fluoresces at $400 \mathrm{~nm}$ is at the origin of this phosphorescence process since these emissions are enhanced in the region adjacent to the SCs.

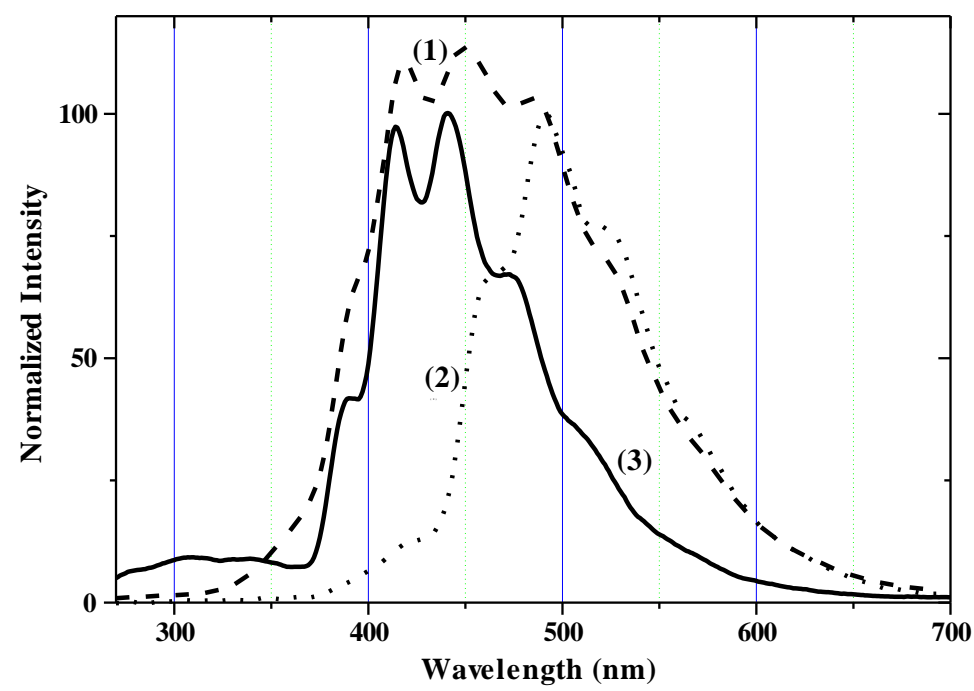

Figure 25. Normalized photoluminescence spectra of samples taken from cable A. Excitation wavelength=250 nm. (1) sample close to inner SC, CW excitation; (2) same as (1)-decay spectrum. (3) isolated sample, taken at $3 \mathrm{~mm}$ from inner $\mathrm{SC}, \mathrm{CW}$ excitation.

\section{IV-3-3-Identification of photoluminescence contributions}

Semi-conducting screens are quite complex materials providing various candidates as diffusing molecules among which ionic impurities associated with the carbon black, acrylate oligomers pertaining to the matrix, antioxidant and crosslinking by-products. For the cable samples under investigation, diffusion of acrylate groups in the insulation has been reported using infrared spectroscopy, with a concentration profile evolving with electro-thermal ageing [68] [69]. However, the structured nature of emission spectra (Figure 25) and their wavelength range are more relevant to phenyl (as is the case for acetophenone) or naphthalene-containing groups. In addition, the concentration profiles of acrylate groups remain sharp close to the semiconductors, even after extensive thermal ageing. So the strategy adopted for unraveling the nature of the new emission was to test different combinations of XLPE/SC interfaces. This was achieved by manufacturing samples constituted of a $500 \mu \mathrm{m}$ thick layer of XLPE cross-linked altogether with a $500 \mu \mathrm{m}$ thick layer of SC of different formulations: the later was taken either in its full formulation, without antioxidant or without cross-linking agent. The respective photoluminescence spectra appear in Figure 26. The $400 \mathrm{~nm}$ emission is clearly present when the SC contains the antioxidant and clearly lacking when no antioxidant is introduced. In addition, the emission appears stronger when peroxide is not introduced, i.e. when the SC is not cross-linked, which might result from a more favorable condition for diffusion when the SC is a pure thermoplastic. In conclusion, these results clearly show that $A O$ from the semi-conducting screens is most probably at the origin of the new features observed in XLPE insulation when coextruded as a cable with the semi-conducting material. As to the evolution of the shape of the profile during thermoelectric ageing, it is difficult to decide if diffusion from the SCs is an ongoing process, or if it results just from a redistribution of the specie once cables have been produced. Luminescence techniques in general have the advantage of being very sensitive to low concentrations of luminescent species; they have the drawback of being delicate to implement for quantification purpose, due to the complex energy exchange processes that may happen, as is probably the case in the complex materials investigated here. 


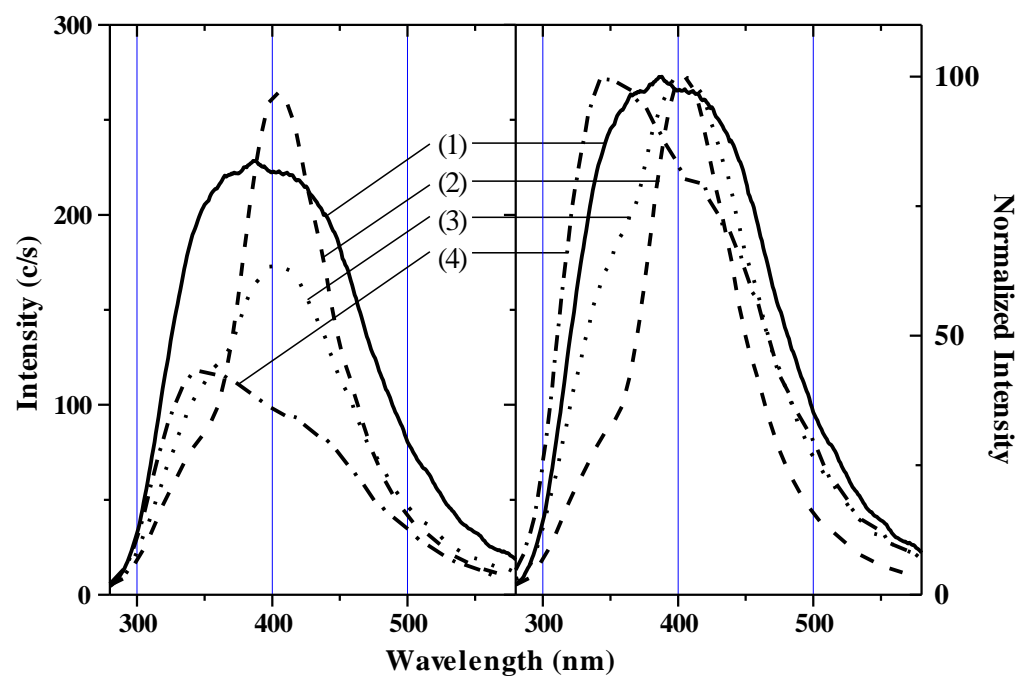

Figure 26. Photoluminescence spectra of lab-compounded samples. (1): XLPE; (2): XLPE/SC without peroxide;

(3) XLPE/SC full formulation; (4): XLPE/SC without AO. In all cases, the full formulation of XLPE was used.

Excitation wavelength $=275 \mathrm{~nm}$. Normalized spectra to the right.

\section{V-Diagnosis, degradation and ageing processes}

\section{V-1-Electroluminescence from LDPE and XLPE}

\section{V-1-1-Rationale for EL measurements}

In the field of electrical insulation, understanding the physical/chemical processes at play during electric ageing and defining transport regimes in which these mechanisms start to be critical is a prime goal to prevent degradation and to develop new formulations or new materials with improved properties. It is thought that a way to define these critical regimes is to investigate under which conditions (in terms of stress parameters) light is generated in the material by excitation with an electric field [53] [54] [70].

This can happen through impact excitation/ionization involving hot carriers or upon bi-polar charge recombination [71]. Although structural and chemical changes induced by hot electrons have often been evoked in electrical ageing [72] [73], a clear evidence of their existence in polymeric insulation is still lacking. The other excitation mechanism of EL is encountered when bipolar charge domains spatially interact, leading eventually to radiative charge recombination.

The question of charge interaction with materials goes with questioning about the way excess energy is dissipated into the materials and whether the dissipative pathways have some irreversible routes. Luminescence naturally reflects the optical route of relaxing energy, besides the phonons route and thermal energy release. The excess energy dissipated in this way - called electroluminescence (EL), is analyzed in multiple ways as it couples the information on the electrical side (charge, current, field) to molecular features (emission spectra). Once excited states have been formed by impact or recombination, they can decay radiatively because the energy of the electronic excitation is of the order of a few electron-volts, i.e. in the range of the visible wavelengths. One can further distinguish between two main classes, i.e. physical versus chemical relaxation pathways, see Figure 27. The balance between these two mechanisms will determine the relationship between EL emission and electrical degradation. 


\section{Localized Excited State}

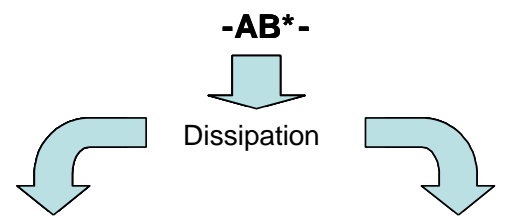

Physical pathway: reversibility

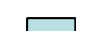

Radiative and non radiative relaxation to the ground state

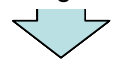

-AB-
Chemical pathway: irreversibility

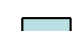

Radiative and non radiative relaxation of byproducts

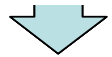

-AC- + -DA-

Figure 27. Schematic representation of the ageing reactions. A: repeat unit of the chain,

$B$ : chain defect, $C$ and $D$ : new chemical functions.

Along the physical pathway, a molecule $A B$ is excited in a non dissociative state $A B^{*}$ and returns to its ground state by a purely reversible effect. The relaxation can occur through a number of radiative (fluorescence and/or phosphorescence) or non radiative (heat dissipation, quenching) processes. The probability of these different pathways is determined by the nature of the excited molecule, its environment, and other experimental parameters such as the temperature and the presence of gas able to act as reaction partner. The transfer of the excitation to an oxygen molecule, which is able to induce further reactions, is obviously important for ageing. Energy transfer can be very efficient in polymers and thus the luminescence spectrum is not always related to the species initially excited. If EL resulted only from physical processes, it would be a way to probe the excitation rate, but its spectral shape would not give any direct information on ageing.

Along the chemical pathway, the excited group dissociates into molecular fragments that can be very reactive and lead to excited states of neutral or charged species. If chemical processes were involved in EL, specific features of the EL spectrum would be expected with possible signatures of degradation products providing a way to measure the dissociation rate, i.e. the ageing rate.

\section{V-1-2-Field regimes of light emission - AC vs DC EL}

Figure 28 shows EL vs field characteristics obtained for XLPE samples either under $50 \mathrm{~Hz}$ AC or DC stress. The presence of by-products of cross-linking reaction led to time-dependent characteristics if the materials were not stabilized before being tested. Samples were consequently thermally treated at $50^{\circ} \mathrm{C}$ for $48 \mathrm{~h}$. Semitransparent gold layers were subsequently deposited by cold sputtering on both sides of the films.

For AC characteristics [74], we have used a short wavelength pass filter with a cut-off wavelength of $600 \mathrm{~nm}$ enabling to get rid of emissions in the red region of the spectrum and which appears as a response of gold electrodes [75]. The characteristic was tentatively fitted following a Schottky-type dependence of EL vs field and the result is represented as a dashed line:

$$
E L \propto \exp (\alpha \sqrt{E})
$$

The AC field threshold is found at about $10 \mathrm{kV} / \mathrm{mm}$ (rms value). In case of DC stress [58], the field threshold is much larger, about $60 \mathrm{kV} / \mathrm{mm}$. Increasing the field up to 120 or $150 \mathrm{kV} / \mathrm{mm}$ still gives a very small signal in case of polyethylene materials. Under AC, higher signal can be obtained, with levels of several $100 \mathrm{~s}$ of counts per second, which makes it possible recording spectra of the emitted light. For these reason EL spectra shown in the following were obtained with AC stress. The reason for having more light intensity under AC arises from alternate injection of charges at one electrode and subsequent recombination. For DC stress charges need to move through the insulation with carriers of opposite polarity generated at the outer electrode. Modelling and analysis of the EL-phase pattern and space charge measurements indicated that very probably the gross of 
recombination processes occur in a region of less than $1 \mu \mathrm{m}$ away from the electrodes while for DC stress space charge measurements show the existence of positive and negative charges in the bulk of the insulation with the possibility of charge recombination.

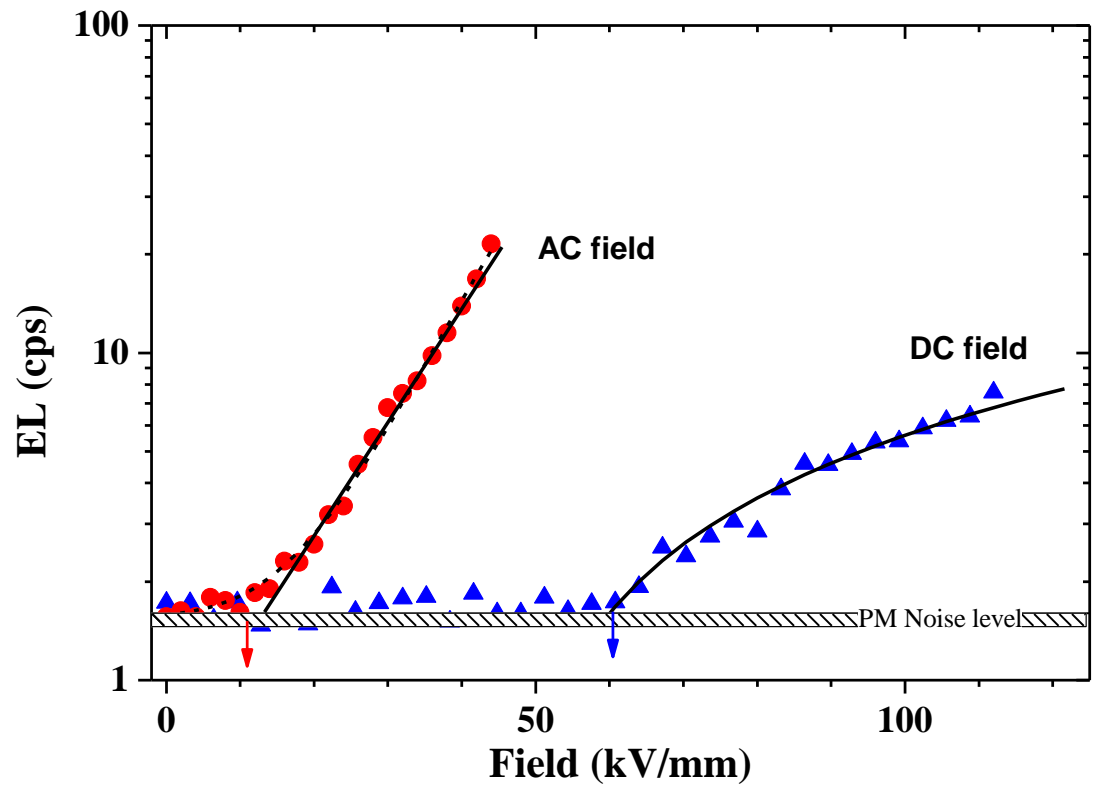

Figure 28. Electroluminescence vs. field characterististics obtained from press-molded films of typically $150 \mu \mathrm{m}$ thickness. In red: $50 \mathrm{~Hz}$ AC stress (rms field). The dashed line is a fit to a Shottky-type law. In blue: DC stress. The line is a fit to linear dependence of EL vs. field above a threshold. Cps stand for photomultiplier counts per second. Data taken from [74] [58].

\section{V-1-3-Electroluminescence spectra}

Comparing spectra obtained in different materials can be a way to improve our understanding of related excited groups. Figure 29 compares the EL spectra obtained on LDPE and XLPE, and for one of the other, the effect of outgassing, of changing electrode material or of film thickness. The results in the ensemble show no great difference from one sample to the other: looking at a glance, the main peak in the spectra is found at about $570 \mathrm{~nm}$, and a clear shoulder appears at about $450 \mathrm{~nm}$, plus a discernible band at $510 \mathrm{~nm}$.

In detail, regarding the effect of electrode material, we have shown that there are two contributions in the EL spectrum under ac stress: one related to the investigated material and the other, in the red region, that has been interpreted as the relaxation of surface plasmon in the metallic electrode [75], [76]. We don't discuss these effects here as they are beyond the scope of this book. After correction for this contribution we can deduce that the spectra are roughly the same, i.e. qualitatively they do not depend on the electrode metal.

The spectra obtained markedly differ from those presented previously, being photoluminescence or RIL spectra. First fluorescence is not detected at all, neither LDPE nor XLPE. However, the emission in the range 430-460 $\mathrm{nm}$ could be related to phosphorescence of the material (even though it is not detected at room temperature in $\mathrm{PL}$ ). The fact that under long treatment of XLPE under vacuum makes this contribution grows could be an indication of phosphorescence contribution. However, as will be seen later on, this emission is related to chemiluminescence of PE that is consistent with a decrease of its contribution with outgassing leading to a decrease of the oxygen concentration inside the film. Considering other materials like poly(ethylene naphthalate) or epoxies for example, we have always found EL from insulation in the long wavelength range compared to the photoluminescence spectra. This makes a strong distinction compared to the PL in organic semiconductors where both singlet and triplet states relaxation is found. It seems therefore that in the EL of insulating polymers mostly lowest lying exciting states, i.e. triplet states are excited. The shoulder at $510 \mathrm{~nm}$ may have relation to the RIL spectrum too. What is noticeable is the remarkable similarity 
of the spectra for XLPE and LDPE, as if the signatures were not influenced by the presence of by-products and more characteristic of the main polymeric chain defects.

The main band in the spectrum with a peak at $570 \mathrm{~nm}$ is not characteristic of the photophysical properties of LDPE or XLPE. As discussed in the following, a hypothesis is that it reflects modification of the materials under the field through the emission from new chemicals formed in the excited state.
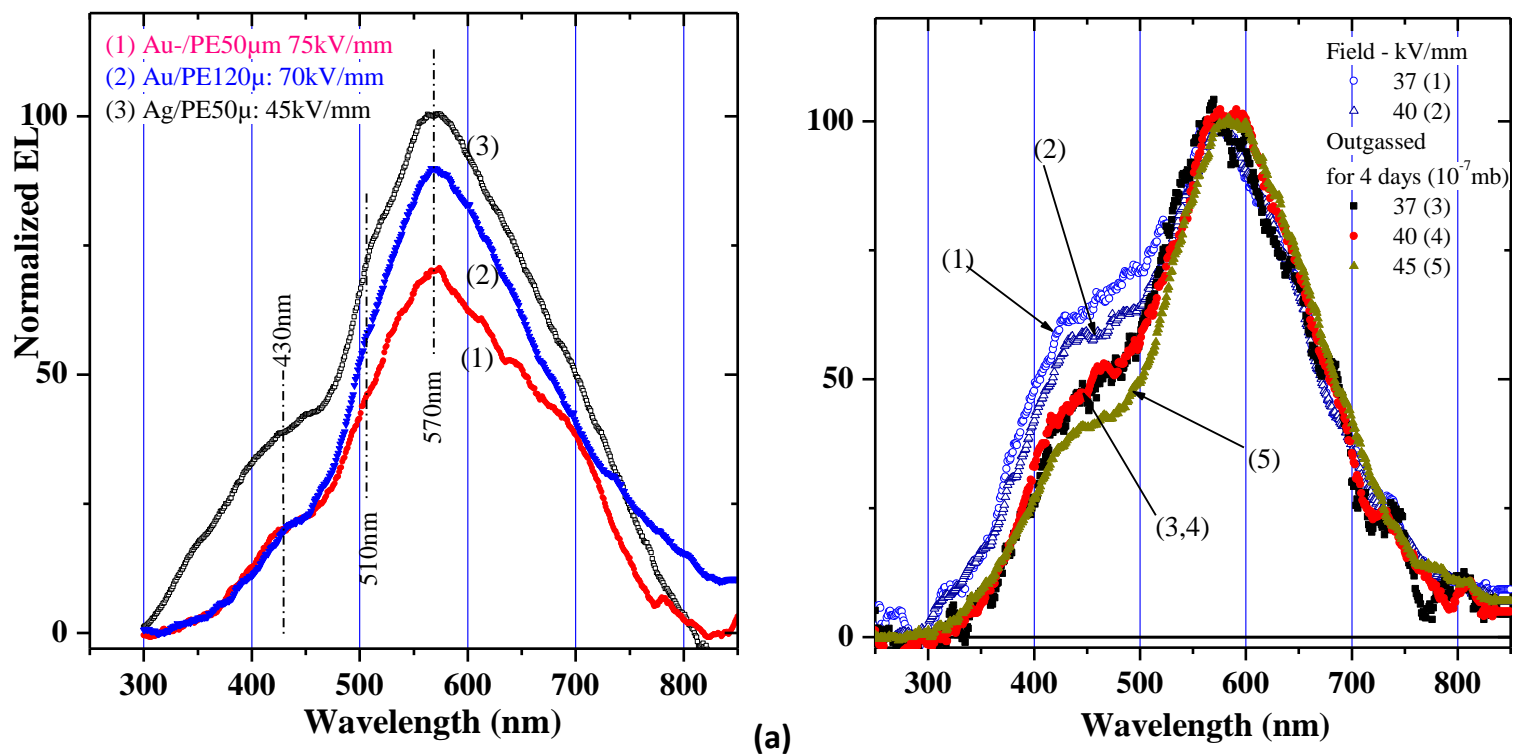

Figure 29. Electroluminescence spectra of LDPE (a) and XLPE (b) obtained in various conditions.

(a): Effet of metal electrode, film thickness and electric field on the EL spectrum of LDPE. See [75].

(b) EL from XLPE from cable peelings, $150 \mu \mathrm{m}$ thick and Au metallization.

The effect of outgassing of peelings not initially thermally treated is put forward.

\section{V-2-Cathodoluminescence as a prototype source for interpreting EL spectra}

A real challenge in the results presented above is the identification of the origin of the main band in EL. With the different ways the material was excited, PL, including PL of oxidized PE, PIL, and chemiluminescence during thermal oxidation, never such emission was revealed. Only with e-beam excitation was it reproduced [15] [77] [78]. It led us to consider electron beam as a prototype source for understanding the EL mechanisms with a much more yied than can be provided under electric field.

\section{V-2-1-The CL spectra from polyolefins}

Figure 30 shows the cathodoluminescence spectra obtained on LDPE and on XLPE with changing the electron beam energy from $2 \mathrm{keV}$ to $10 \mathrm{keV}$. With this energy, the penetration depth of electrons is less than $1 \mu \mathrm{m}$. The experiments were achieved with the same beam current; only the energy of the electrons was changed. The amplitude of the peaks is grossly proportional to the energy deposited by incident electrons. In the time scale of the measurements, of the order of $10 \mathrm{~s}$ at each energy level, we didn't observe significant evolution of the spectrum. The EL spectrum obtained under AC stress is added for comparison.

Two main obervations can be made. The first one is that the EL and CL spectra show obvious similarities, much more than any of the spectra shown in the previous sections. Grossly, there are 3 main bands located at $\approx 420 \mathrm{~nm}$, at $510 \mathrm{~nm}$ as a shoulder, and at $570 \mathrm{~nm}$ (main band). The shoulder found at $700 \mathrm{~nm}$ in the EL spectrum of LDPE is more probably related to the treatment done in removing the red component -surface plasmon related - in the EL spectrum [75]. The three main processes are interpreted as a superposition of chemiluminescence from the material, a contribution from charge recombination and a main band associated 
to the formation of by-products under the effect of energy deposition. The spectra are analysed in more details in the next section.

The second feature is the similarity between the results obtained for LDPE and XLPE response and this is consistent with the comparison of the EL features. So again, while residues, some of them aromatics, drive the photophysical properties of XLPE, it is apparently the main chain of the polymer that drives the luminescence response under electron irradiation.
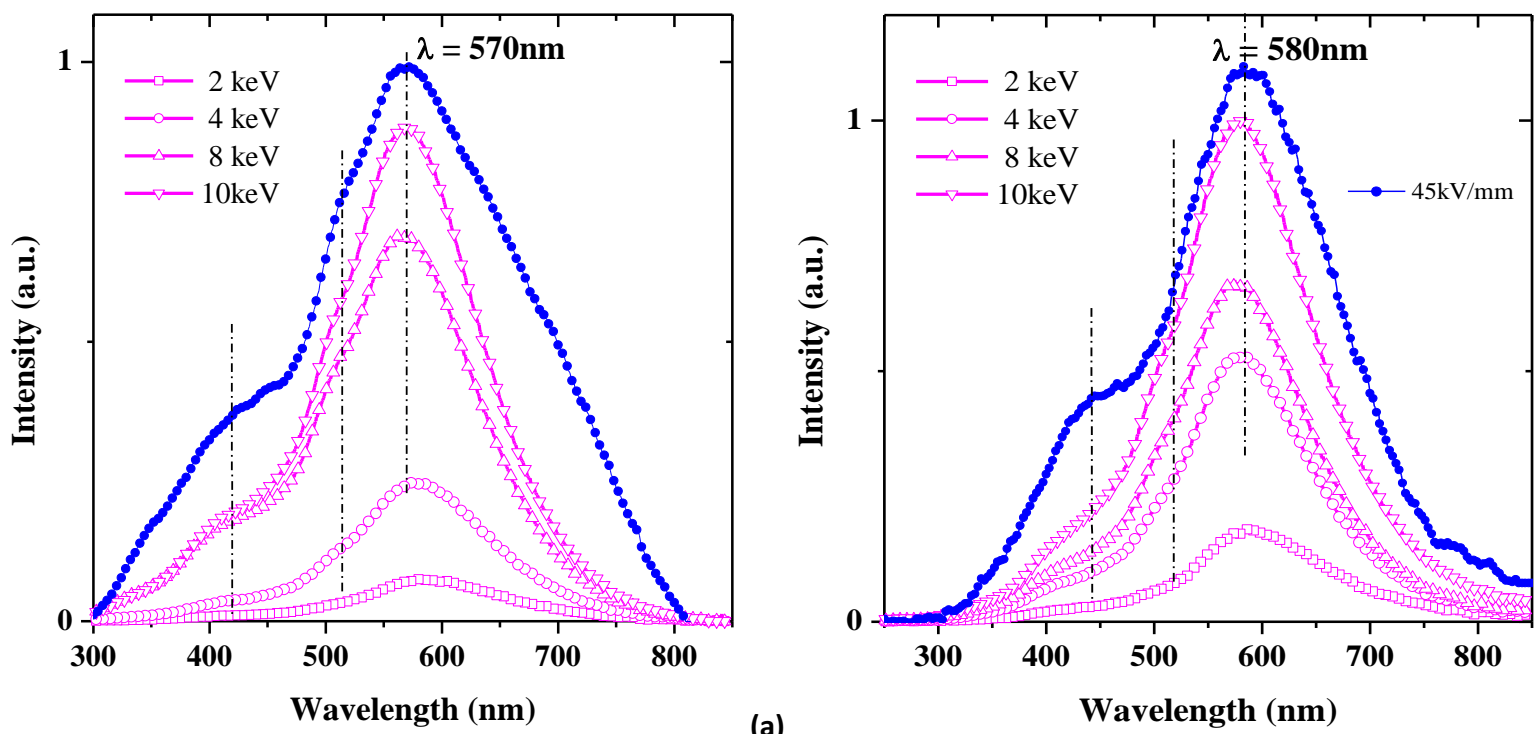

(a)

Figure 30. Cathodoluminescence spectra of (a) LDPE and (b) XLPE under different e-beam energy.

EL spectra given for comparison, for fields of $75 \mathrm{kV} / \mathrm{mm}$ (LDPE) and $45 \mathrm{kV} / \mathrm{mm}$ (XLPE) [77].

The similarity between EL and CL spectra leads to the conclusion that EL involves material degradation, like has already been reported in case of poly(ethylene naphthalate) [79]. The signatures of charge recombination and chemiluminescence seen in the EL spectra of polyethylenes support the existence of these mechanisms under electrical stress. They can be a direct consequence of hot electron impact with the creation of reactive species (which could further react with oxygen molecules dissolved in the amorphous fraction of the polymer) and the generation of secondary electrons (which would then be trapped and available to recombine with trapped holes at low to moderate field). The extra spectral features revealed in $\mathrm{CL}$, which provide a full understanding of the EL spectrum, are likely linked with a specific pathway bound to electronic impact. The use of a $10 \mathrm{keV}$ electron beam energy could appear too far from the energy of hot electrons in polymers ( $<10 \mathrm{eV})$ to be typical. However, one has to keep in mind that interaction between electron beam and matter leads first to a decrease of energy of the individual electrons by X-ray emission (Bremsstrahlung) before they efficiently start their interaction with valence electrons and localized centres in the gap. It follows a set of possible excitation mechanisms among which impact ionization at moderate kinetic energy (order of $15 \mathrm{eV}$ ), impact excitation $(10 \mathrm{eV})$, quasielastic scattering with phonons (energy $<10 \mathrm{eV}$ ), and finally electron trapping at thermal energy [80]. All these events are those that are thought to be important under electric field.

\section{V-2-3-The CL spectrum decomposition into different processes}

Results discussed above show that EL and CL from polyethylenes have rather generic signatures, not obviously dependent on the type of PE. The finding was further substantiated by considering $\mathrm{CL}$ and $\mathrm{PE}$ in bioriented polypropylene (BOPP), which is another polyolefin. The same generic features were obtained, which led us to decompose each spectrum ( $\mathrm{CL}$ or $\mathrm{EL}$ ) into a sum of 'elementary' contributions, represented by spectra discussed in the previous section [76].

The obtained results are summarized in Figure 31. The reference bands for fluorescence and RIL do not need to be further commented as they have already been described before. For chemiluminescence, we refer to chemiluminescence spectra obtained along thermal oxidation at the earliest stages of the process as measured 
during the oxidation of polypropylene [8]. This contribution was already used to interpret the different contributions in plasma-induced luminescence experiments [27]. Note that for PIL, there was an evolution of the spectra during the decay time. Here in case of polyolefins the $\mathrm{CL}$ spectrum did not evolve significantly when changing the irradiation energy or during the irradiation time though the intensity droped in a matter of minutes during irradiation. Therefore, the change in spectrum shape could not guide the interpretation of the processes. For EL, in the same way it is not easy to reveal relative changes in contributions in the case of polyolefins. For PEN, there were indeed significant field effects in spectrum shape [81].

\section{(a) Elementary signatures}

- FL: Fluorescence at $328 \mathrm{~nm}$

- $\mathrm{CHL}$ : Chemiluminescence at $415 \mathrm{~nm}$ (identified in PIL and during thermal oxidation of PP)

- RIL: main contribution in recombinationinduced luminescence of LDPE or PP

- Peak4: characteristic of degradation byproduct, peaking at $573 \mathrm{~nm}$

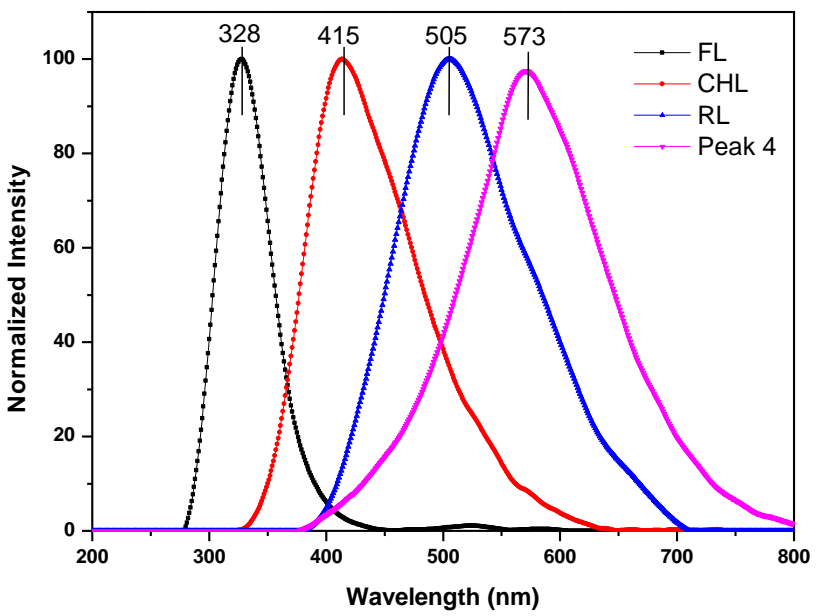

(b) CL spectrum of XLPE

- Main contributions from degradation byproduct $(91.5 \%)$ and from chemiluminescence ( $8 \%$ of area under the luminescence spectrum)

- Comparatively more RIL (1\%) and CHL (25\%) relative contribution in BOPP

- Good fit of the envelope

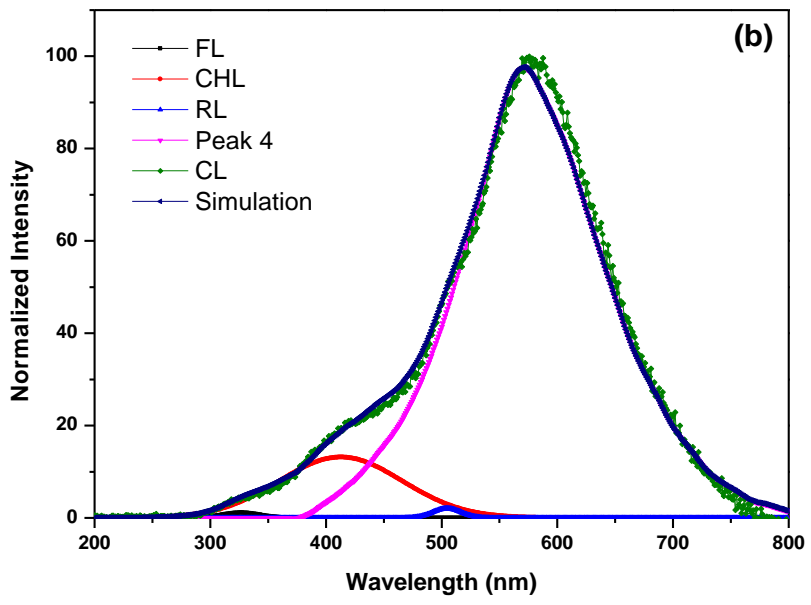

\section{c) EL spectrum of LDPE}

- Clear effective contribution from RIL (8.5\%) as well as in BOPP (5.3\%)

- Chemiluminescence represents $11 \%$ vs $7.7 \%$ in BOPP

- Excellent fit of the envelope

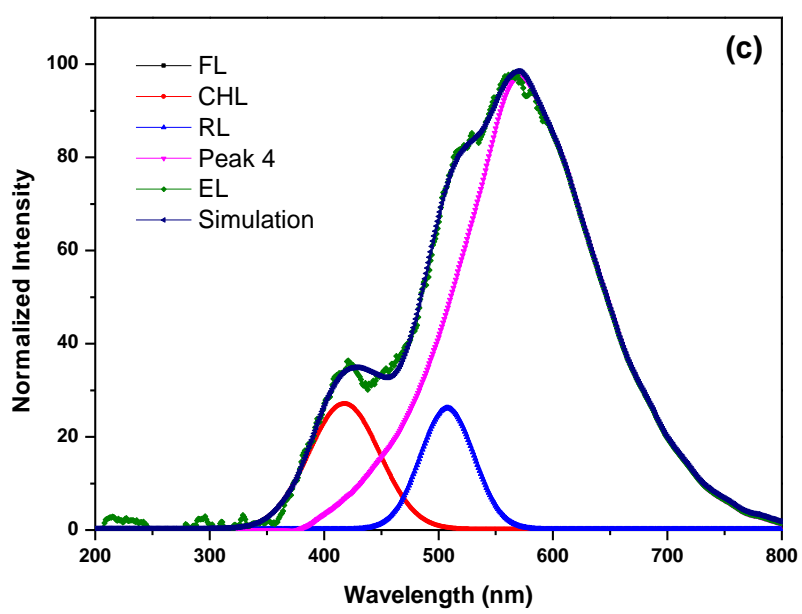

(c)

Figure 31. Decomposition of EL and CL spectra of PE into elementary contributions and examples of application. (a) CL in XLPE (5 keV energy) and (b) EL in LDPE (ac field $45 \mathrm{kV} / \mathrm{mm}$ ). Adapted from [76]. 
In $\mathrm{CL}$ the kinetic energy of electrons is a powerful source of excitation. The main part of the energy of the electron is spent during deceleration on the production of secondary electrons and plasmons with energy of 20-30 eV. Secondary electrons that do not leave the sample in turn generate electron-hole pairs. Thus, the main fraction of the electronic energy (up to 90\%) is spent on the creation of electron-hole pairs [82]. It is important to note that, upon excitation of luminescence by an electron beam, the energy of excitation considerably exceeds the bandgap width of the material. Therefore, excitation of luminescent centers does not result only from direct excitation but also as a result of radiative and nonradiative transitions from higher lying energy states. The main primary mechanism of luminescence excitation will be impact excitation on the molecules of the polymer. The energy exchange during the impact may give rise to excitonic states that will further relax to their ground state (generating fluorescence and/or phosphorescence emission) or be brought into dissociative states. The formation of new chemical species upon degradation is therefore inherent to $\mathrm{CL}$. Alternatively, transient excitonic states may react with oxygen molecules dissolved in the polymer, particularly in the case of a triplet exciton taking into account the triplet ground state of oxygen molecule. It follows the possibility to emit light through chemiluminescence. Impact ionization will be a source of positive charges and there is the possibility of charge recombination generating light. In recombination processes, the emitting species will be those able to act as recombination centers i.e. those providing deep traps for electric charges. In polyolefins, these centers are conjugated CC bonds bound to $\mathrm{CO}$ species. Owing to the chemical degradation of the material under the electron beam all these recombination processes can occur on by-products leading to new components in the spectra. CL experiments provide therefore a full set of excitation channels.

In EL, the source of excitation is much less efficient and is still the topic of debates in large band gap materials [42]. Hot electrons processes and charge recombination are the main potential sources of excitation, depending on the electric stress. In case of hot electron processes [73] and although the kinetic energies are far apart from that in $\mathrm{CL}$ experiments (a few eV in $\mathrm{EL}$ vs. a few keV in $\mathrm{CL}$ ), there is still the possibility for impact processes, with or without ionization. Impinging electrons with kinetic energies of a few eV do not have the energy required for impact ionization (15 eV) [83] but they can excite species among which the natural chromophores that will relax through fluorescence and phosphorescence emission, or be stabilized to form transient ions (negative attachment) able to subsequently dissociate into molecular fragments [84]. Recombination between charges of opposite polarity being injected at cathode and anode also provides the conditions for EL in polyolefins [42]. It has to be realized that recombination opens the way to chemiluminescence as well through the excitonic states created by the recombination process itself that can react with dissolved oxygen. Because a chemical pathway is open through reactive excited species, recombination can further affect newly formed chemical groups.

The main component in the emission in CL and EL has not been identified. We think it is a signature of material degradation either through the formation of by-products under an excited form or through their excitation upon charge recombination. Indeed, the fate of excitons formed upon recombination of electron/hole pairs has been investigated in PE using density functional calculations and ab-initio molecular dynamics simulations. Two situations were investigated where excitons are self-trapped along a chain [85] [86] or trapped at chemical defects [87]. The relaxation can occur following different pathways depending on the case. When the exciton is trapped on a chemical defect, the relaxation pathway depends of the nature of the chemical defect leading to trapping of the charges, non-radiative recombination or homolitic bond-breaking. This is of course the latter process that is relevant for damage. When the exciton is self-trapped along a chain, $\mathrm{CH}$ bonds breaking is promoted according to a recent calculation [87]. With the opening of a chemical route, by-products could be produced in the excited state and be responsible for emission in a wavelength range that is not typical of the initial chemistry of the polymer. The main peak in electroluminescence could reveal such degradation process. 


\section{V-3-Ageing diagnosis}

Besides on-line light detection under application of the stress as presented above, luminescence can be used as post-ageing diagnosis method like any other physicochemical analysis, with its own specificities as the strong dependence of the sensibility upon the nature of molecular groups. In general, the trends are not an increase of luminescence, as could be expected with the formation of new groups. Rather, the luminescence yield decreases with ageing and the shape of the spectrum exhibits modification like broadening and shift to long wavelength. This is typically the case during thermal oxidation of polyolefins.

\section{V-3-1-EL yield of aged materials}

A series of experiments has been achieved concerning EL-field characteristics of aged XLPE. Mary et al. showed that upon prestressing of materials under DC stress, the phase patterns under AC stress are considerably modified [88]. This is a consequence of space charge build-up of the material that can be viewed as (possibly reversible) ageing.

In the frame of the Artemis project, cable insulation slices were characterized using EL, through EL-field characteristics under AC and DC. The trend was an increase in the field threshold with ageing (mostly thermal ageing), along with a decrease in the slope of the characterisitic. Figure 32 shows an example of EL-field characteristics under DC stress. The cables had a $1600 \mathrm{~mm}^{2}$ copper conductor and a $26.6 \mathrm{~mm}$ thick XLPE insulation. One of them was aged at different levels of field and temperature (maximum test field $=27.5 \mathrm{kV} / \mathrm{mm}$, withstood for 1 year, maximum temperature $95^{\circ} \mathrm{C}$ ). The field threshold is significantly shifted to higher value. This was correlated to space charge measurements showing that the recombination domain in aged cable is forming at a higher field than in unaged, due to a smaller penetration depth of positive space charges. These changes were explained by an increase in density and energy distribution of trapping centres [89]. The photoluminescence yield was halved after ageing.

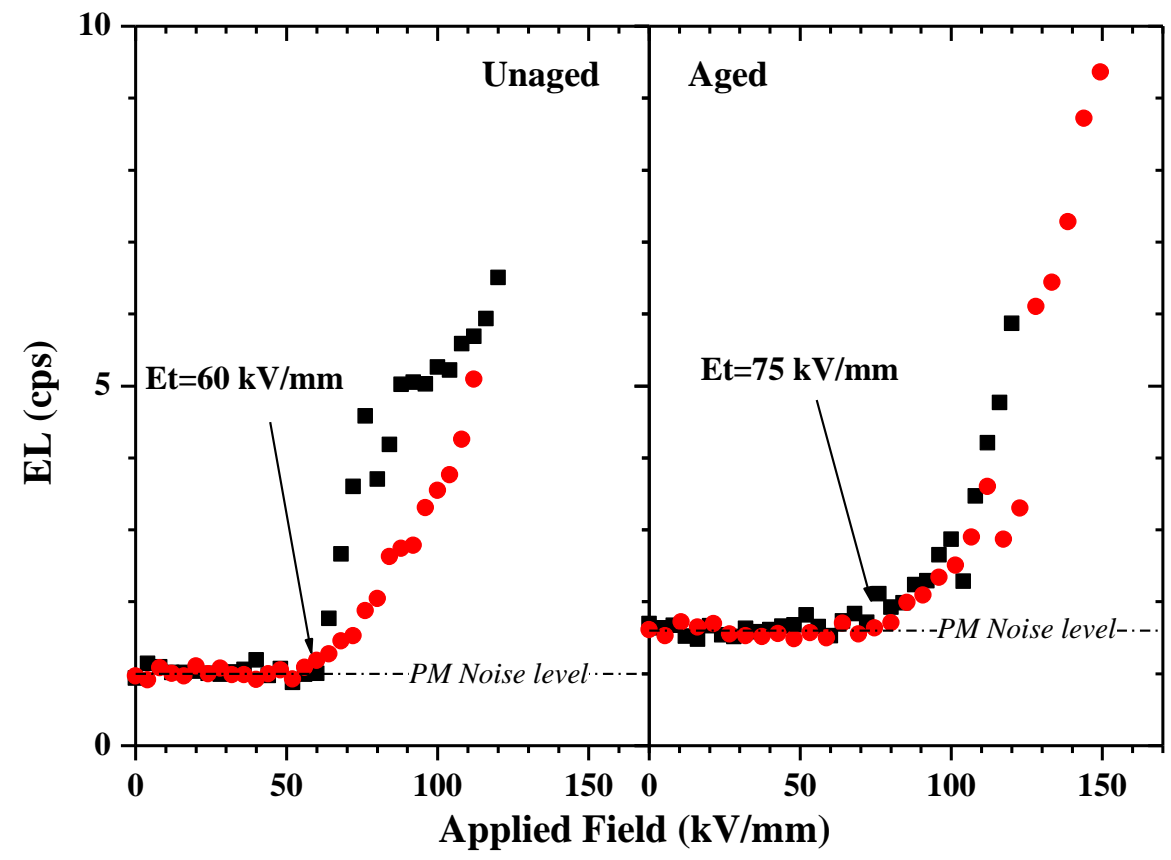

Figure 32. EL-field characteristics from XLPE peelings taken from reference and aged cable in Artemis project. Aging condition: $95^{\circ} \mathrm{C}$, $\max$ ac field $27.5 \mathrm{kV} / \mathrm{mm}, 1$ year [89]. 


\section{V-3-2-PL from thermally degraded materials}

Still within the Artemis project, the photoluminescence was used to monitor the changes in material property with ageing time. Already some results have been shown in section IV-3-2. The diffusion of antioxidant from the semiconductor to the insulation has been followed by luminescence. AO diffusion was not the only process at play. Cable yellowing was detected and this was correlated to the appearance of extra band in photoluminescence spectra [55] [56]. The analysis of the PL data revealed quite complex shape of PL spectra, with several overlapping processes. As each contributing band is usually broad, monitoring the intensity of the emission related to one mechanism is not possible since significant overlap of the bands occurs. In order to provide a systematic evaluation of photoluminescence spectra vs. position in the roll, ageing conditions, and excitation wavelength, a set of elementary spectra was isolated. The identification of those isolated bands had also to take into account the fat that XLPE ws produced with two different companies with different crosslinking process which gave significant differences in PL signatures. The identified bands, their characteristics and significance are given in Table 3. The fluorescence bands are sorted out in three groups:

i/ those related to XLPE, with emission maxima at 330, 370 and $440 \mathrm{~nm}$. The details of these bands depend notably on the excitation wavelength.

ii/ those associated with the antioxidant of the SC screens: the main band is at $400 \mathrm{~nm}$; it may shift to $410 \mathrm{~nm}$ after thermal ageing. Finally, a small fluorescence band at $340 \mathrm{~nm}$ is found in some cases, with more amplitude close to semicons.

iii/ the last emission, at $540 \mathrm{~nm}$ is detected after significant thermal ageing, and appears correlated to the yellowing of the cables. The spectrum is very broad with a width at half maximum of about $200 \mathrm{~nm}$ while that of the XLPE-related fluorescence is less than $100 \mathrm{~nm}$. This broadness reflects probably the disorder introduced in the related chemicals.

Some resuls obtained after fitting are plotted in Figure 33. The contributions to the spectra for the 3 groups of processes, XL for bulk XLPE, SC for diffusing species from screen and $Y$ for yellowing were used summed up. We consider here specifically the behavior of the AO-related band (labeled SC) and yellowing (Y) band in one type of cable (B). As electric field ageing effects are of second order compared to thermal ageing, we indicated in the caption only the ageing times at $90^{\circ} \mathrm{C}$ for this set.

\begin{tabular}{ccc}
\hline $\begin{array}{c}\text { Label - } \\
\text { Peak wavelength }(\mathbf{n m})\end{array}$ & $\begin{array}{c}\text { Area of the band } \\
(\mathbf{n m})\end{array}$ & Attribution \\
\hline XL-440 & 87.1 & bulk XLPE emission \\
XL-370 & 87.0 & bulk XLPE emission \\
XL-330 & 44.8 & bulk XLPE emission \\
SC-400 & 100.2 & AO from SC \\
SC-410 & 90.6 & AO from SC+ ageing? \\
SC-340 & 45.2 & AO from SC \\
Y-540 & 175.5 & thermal ageing \\
\hline
\end{tabular}

Table 3. List of Fluorescence signatures isolated in cable insulation with the corresponding attribution. The area of the bands is given for spectra normalized to 1 at peak intensity.

The profiles presented in Figure 33.a for the SC-related band differ significantly from those shown in Figure 24.a. There reason is with the cable processing conditions which were different between the two cables, revealing that the chemical disorder in XLPE struture remains process dependent. Without thermal ageing (rolls BO to $B 2$ ), the relative contribution of the yellow band is perfectly reproducible from one roll to another (though it was not so clear for Cable A). After thermal ageing, the profile becomes more flat and the intentity in the middle of the sample grossly increases with ageing time. Concerning the band related to yellowing, Figure 33.b, a maximum contribution is found close to the inner semicon, where the ageing temperature was at maximum. In consequence, the profile is no more symmetrical. 
UV-vis absorption spectra were correlated to PL measurements. A broad absorption appears in the region 350$450 \mathrm{~nm}$, strong close to the inner SC and decreasing in intensity with the distance from the inner SC. It appeared related to a thermal effect during ageing since the temperature is the higher at the core of the cable and decreases when going towards the outer SC. It was suspected that the photoluminescence emission at 540nm and the broad absorption in the range 350-450 $\mathrm{nm}$ are associated with the same kind of species formed by thermal oxidation. The apparent yellowing would be mainly due to the absorption. As to the nature of reacting groups, one has to consider the involvement of AO-related species that have diffused from SC to the insulation bulk during cable production. As antioxidant agent, such species are prone to reaction with oxygen during thermal ageing, leading potentially to the absorption responsible for the yellowing. Further support to the interpretation was brought by comparing the behavior of cables $A$ and $B$ and by considering the profiles of the diffusion and yellow bands [56].

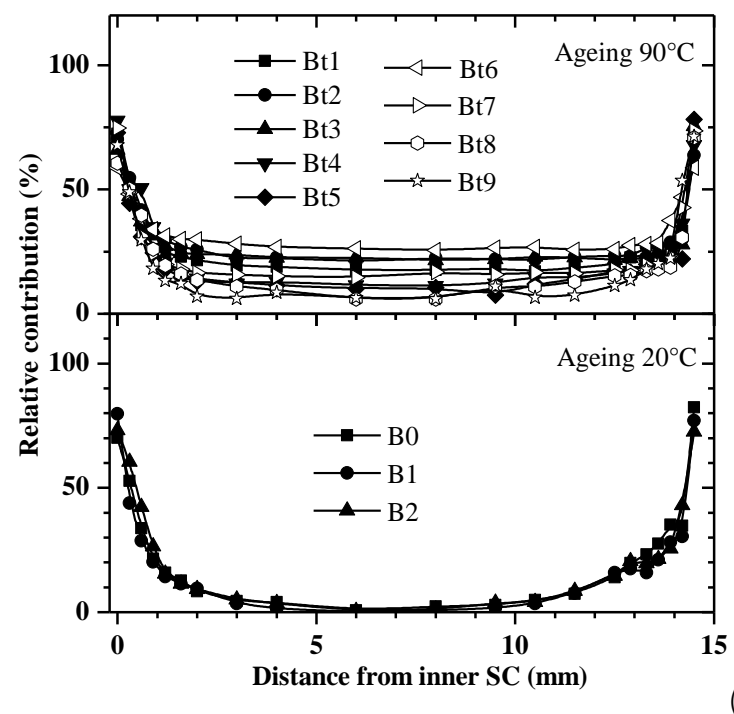

(a) SC-related band

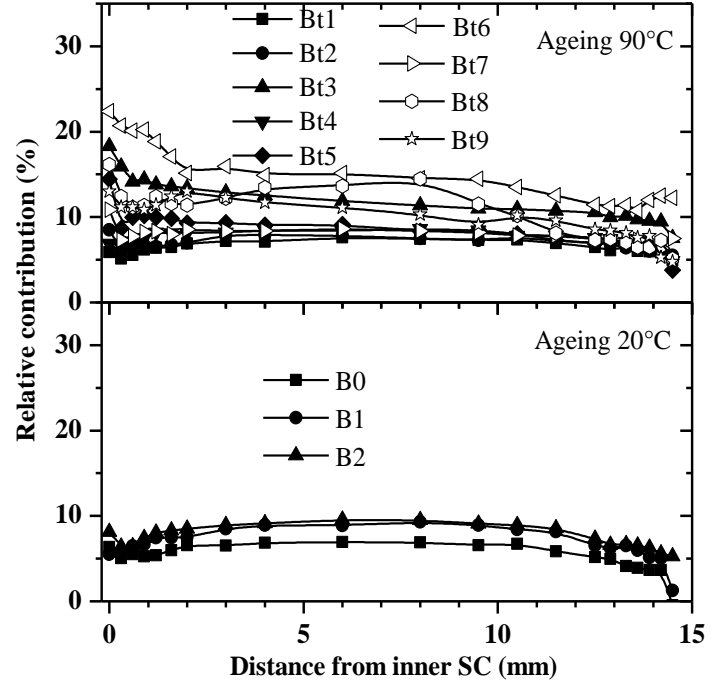

(b) Yellow band

Figure 33. Relative contribution of the SC-related and Yellow bands to the total PL spectrum area versus insulation radius for cables B. Ageing times at $90^{\circ} \mathrm{C}$ : Bt5: $3000 \mathrm{~h}, \mathrm{Bt1}, 2$, and 4: $5000 \mathrm{~h}$; Bt9: $6000 \mathrm{~h}$;

Bt7: 7800 h; Bt3 and 6: 10000 h; Bt8: 15000 h.

\section{V-3-3-Contact sensitization}

As final example of application of luminescence to probe defects into polyethylene, we present the case of LDPE films processed using different cover layers during press-molding [90]. As a base insulation material we used a non-stabilized low-density polyethylene from ExxonMobil ${ }^{\mathrm{TM}}$, which is designed for Medium/High Voltage insulation, and which can be peroxide crosslinked. The films were processed by press-molding at $140^{\circ} \mathrm{C}$, in film forms of 200 to $250 \mu \mathrm{m}$ thickness and using Aluminum film (Al), polyimide (PI) or polyethylene terephthalate (PET) cover layers. Surprisingly, very different PL spectra were obtained dependening on the kind of pressmolding films used in the process, despite the fact that the processing temperature was moderate.

Figure 34.a shows the PL spectra obtained on the various materials, for an excitation wavelength of $280 \mathrm{~nm}$ (the emission spectrum appeared dependent on the excitation wavelength). The bands at $300 \mathrm{~nm}$ and $340 \mathrm{~nm}$ are consistent with previous reports on PL from LDPE, the main band being assigned to enone $-\mathrm{C}=\mathrm{C}-\mathrm{CO}-$ groups [9]. The shoulder at $390 \mathrm{~nm}$ is not so commonly observed in fluorescence of PE. In fact, considering the films made with PET cover layer, one can deduce that this emission is strengthened and its position is consistent with that detected in the PET-film itself. The emission at $390 \mathrm{~nm}$ in PET was ascribed to a ground state dimer emission [91]. 


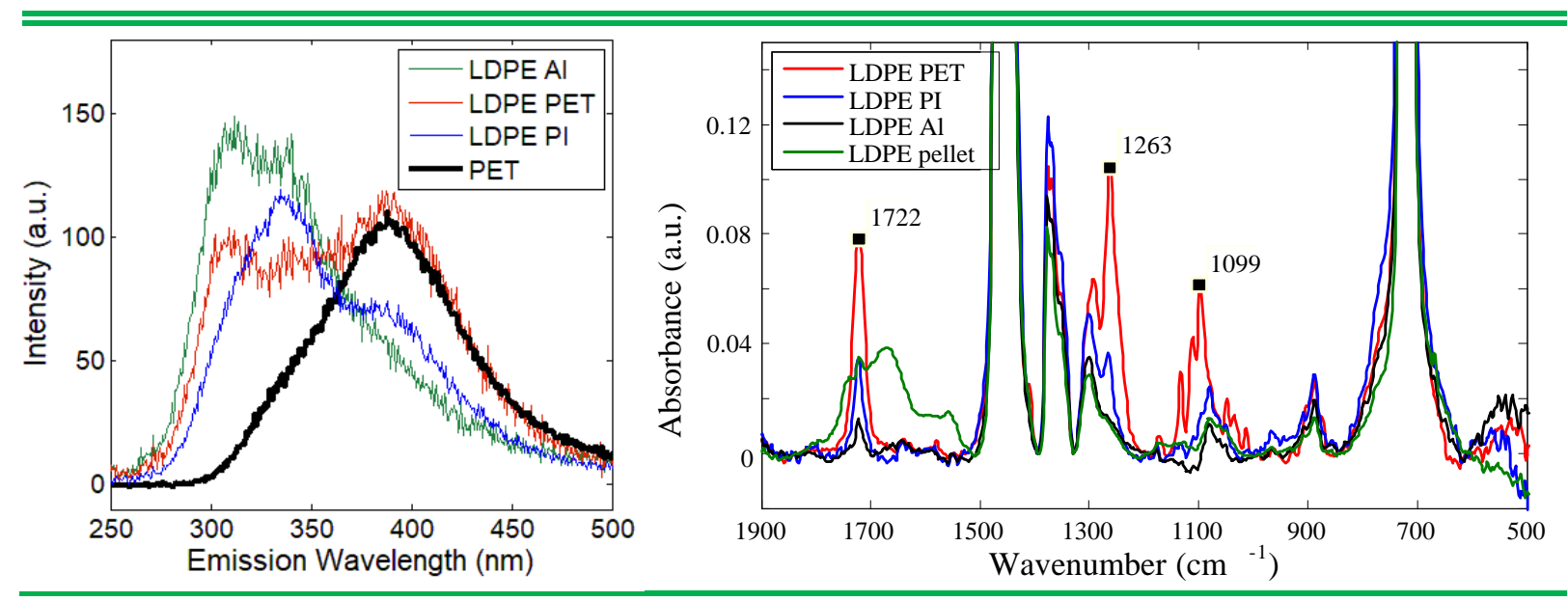

(a) Photoluminescence

(b) ATR-FTIR spectroscopy

Figure 34. (a) PL emission spectra for LDPE processed with Al, PET or PI coverlayers compared to PET film (intensity was divided by a factor 5 for the PET film). Excitation wavelength: $280 \mathrm{~nm}$. (b) FTIR spectra of LDPE pellets and of films processed using different cover layers. ATR mode with internal reflection angle of $45^{\circ}$.

FTIR spectra shown in Figure 34.b feature three main absorption bands changing depending on the type of cover film used. Bands at $1722 \mathrm{~cm}^{-1}, 1263 \mathrm{~cm}^{-1}$ and $1100 \mathrm{~cm}^{-1}$ appear for the sample processed with PET. They are ascribed respectively to $\mathrm{C}=\mathrm{O}$ and $\mathrm{C}-\mathrm{O}$ stretching vibrations and to cyclic ether vibrations. The band at 1645 $\mathrm{cm}^{-1}$ (in the pellets) ascribed to $\mathrm{C}=\mathrm{C}$ groups which disappears after processing, is presumably some kind of process stabilizer.

Both the PL and FTIR data reveal a specific response of the material processed with PET cover-layer. The signatures might be interpreted in terms of oxidation of the material due to the contact with the PET cover layer (e.g. formation of carbonyl groups). This would be consistent with the fact that some of the signatures are detectable in LDPE processed with or without PET, the difference being that the magnitude is higher when contact with PET is made. A second possibility is the diffusion of low molecular weight molecules from the PET to the LDPE during the thermal processing. As PET contains carbonyl groups in the repeat unit, the response of both pre-oxidized groups and of diffusing groups would be combined.

The interpretation given to the extra-signatures was therefore to diffusion of oxidized groups -presumably decomposition products or oligomer chains from PET into the LDPE sub-surface. Substantial differences in space charge [92] and conductivity [93] were reported for LDPE exposed to PET molding layers; the diffusion is one of the possibilities for such differences. Oxidized groups may act on interface properties through different ways: changing the surface potential, acting as trapping sites for charges, or they may ultimately lead to dipoleinduced effects. 


\section{VI-Conclusions}

By using luminescence techniques, we have been able to show that the by-products of cross-linking reactions may act as deep traps in XLPE. This provides an explanation of the dependence of the space charge distribution on the thermal treatment of the samples that has been reported in the literature [94]. These chemicals are not the only trapping centres in XLPE as denoted by the emission spectrum of this last material. Anti-oxidant and its reaction products have been shown to be very active species for stabilizing electric charges. Example has been given where diffusing species from the semi-con screens give their contribution to the photoluminescence emission. A careful treatment of the emission spectra with the help of spectra obtained on model compounds allow to deconvolute the complex response obtained on XLPE.

Examples have been given where luminescence techniques can be usefull to tackle the question of the ageing mechanisms and ageing diagnosis. The interpretation of electroluminescence spectra of PE related materials has only been possible with the help of database related to the emission of model material compounds under different excitation sources, among them cathodoluminescence with its characteristic emission spectrum that maps those of PE related compounds. This has been fundamental in establishing the relationship between electroluminescence and ageing.

The application of luminescence experiments to derive the nature of the recombination centres may suffer from some limitations. First, the light that is analysed may not be emitted directly by the recombination centre, but through energy transfer to an efficient emitter. This would prevent a direct correlation between the emission spectrum and the recombination centre. However, this study has shown that the RIL spectra are indeed linked to the chemicals introduced into the material. This drawback would not therefore be as drastic as is often postulated. Second, different chemicals can give emissions in a narrow range of wavelengths thereby rendering critical the assignment of a particular spectrum to a given species. Moreover, the spectrum can become intractable when dealing with technical materials containing different kinds of recombination centres. This is why knowledge of the optical signature of different additives is of great help.

Finally, the approach is limited to recombination centres that are optically active. This item is difficult to address since the emission yield as probed by photoluminescence as for example is not necessarily the same as in RIL. The requisite condition is that the centres must have a finite probability of emitting light when interacting with electrical charges. In photoluminescence, the probability of creating a triplet state depends on the rate of intersystem crossing from singlet states. It can be very low, especially when the difference in energy between the singlet and triplet states is large. Conversely, the probability of creating a triplet excited state in a charge recombination event is theoretically three times as large as the probability of creating a singlet excited state [95]. This means that species known to be non-phosphorescing may be so because of excitation limitations under uv irradiation and they may behave in a completely different way regarding emission yield excited by electrical charges. Clearly, there are not so many alternative principles for measuring to luminescence that are able to bring direct information on the interaction between electrical charges and chemicals within insulators. This technique can be useful when used in conjunction with different sources of luminescence excitation to check the possible role of additives or impurities in space charge accumulation phenomena, and to probe ageing mechanisms. 


\section{References}

[1] G. Teyssedre and C. Laurent, "Advances in high-field insulating polymeric materials over the past 50 years," IEEE Trans. Electr. Insul. Mag., vol. 29_5, pp. 26-36, 2013.

[2] G. Chen, M. Hao, Z.Q. Xu, A. Vaughan, J.Z. Cao, and H.T. Wang, "Review of high voltage direct current cables," J. Power Energy Systems, vol. 1, pp. 9-21, 2015.

[3] Y. Zhou, S. Peng, J. Hu, and J. He, "Polymeric insulation materials for HVDC cables: Development, challenges and future perspective," IEEE Trans. Dielectr. Electr. Insul., vol. 24, pp. 1308-1318, 2017.

[4] J.C. Fothergill, G.C. Montanari, G.C. Stevens, C. Laurent, G. Teyssedre, L.A. Dissado, and U.H. Nilsson, "Electrical, microstructural, physical and chemical characterization of HV XLPE cable peelings for an electrical aging diagnostic data base," IEEE Trans. Dielectr. Electr. Insul., vol.10, pp. 514-527, 2003.

[5] R.B. Blodgett and R.G. Fisher, "Insulations and jackets for cross-linked polyethylene cables" IEEE Trans. Power Apparatus and Systems, vol.82, pp. 971-980, 1963.

[6] L. Zlatkevich (Editor), Luminescence Technique in Solid State Polymer Research, Marcel Dekker Inc, 1989.

[7] G. Teyssedre, G. Tardieu, and C. Laurent, "Characterisation of crosslinked polyethylene materials by luminescence techniques," J. Materials Sci., vol. 37, pp. 1599-1609, 2002.

[8] P. Tiemblo, J.M. Gomez-Elvira, G. Teyssedre, F. Massines, and C. Laurent, "Chemiluminescence spectral evolution along the thermal oxidation of isotactic polypropylene," Polym. Degrad. Stab., vol. 65, pp. 113-121, 1999.

[9] G. Teyssedre, L. Cisse, C. Laurent, F. Massines, and P. Tiemblo, "Spectral analysis of optical emission due to isothermal charge recombination in polyolefins," IEEE Trans. Dielectr. Electr. Insul., vol. 5, pp. 527-535, 1998.

[10] G. Teyssedre, C. Laurent, G. Perego, and G.C. Montanari, "Charge recombination induced luminescence of chemically modified cross-linked polyethylene materials," IEEE Trans. Dielectr. Electr. Insul., vol. 16, pp. 232240, 2009.

[11] N. Shimizu, H. Katsukawa, M. Miyauchi, M. Kosaki, and K. Horii, "The space charge behavior and luminescence phenomena in polymers at 77 K," IEEE Trans. Electr. Insul., vol. EI-14, pp. 256-263, 1979.

[12] T. Lebey and C. Laurent, "Charge injection and electroluminescence as a prelude to dielectric breakdown," J. Appl. Phys., vol. 68, pp. 275-282, 1990.

[13] H. Yamamoto, M. Mikami, and S. Nakamura, "Nonlinear cathodoluminescence from insulators," J. Lumin., vol. 102-103, pp. 782-784, 2003.

[14] L..J. Brillson, "Applications of depth-resolved cathodoluminescence spectroscopy," J. Phys. D: Appl. Phys., vol. 45, p. 183001, 2012.

[15] G. Teyssedre, J.L. Franceschi, and C. Laurent, "Cathodo- and electro-luminescence spectra in insulating polymers: a parallel approach for inferring electrical ageing mechanisms," Proc. Conf. Electrical Insulation and Dielectric Phenomena (CEIDP, Vancouver, Canada), pp. 824-827, 2007.

[16] J. Hagekyriakou and R.J. Fleming, "Determination of the kinetic order of thermoluminescence in the presence of a distribution of electron trap activation energies," J. Phys. D: Appl. Phys., vol. 15, pp. 163-176, 1982.

[17] A. Markiewicz, D.V. Balbachas, and R.J. Fleming, "Simultaneous thermally stimulated luminescence and depolarization current in low density polyethylene," J. Thermal Analysis, vol. 37, pp. 1137-1152, 1991.

[18] T. George and K.J. Randle, "The isothermal luminescence and thermoluminescence of gamma-irradiated polyethylene at 77 K," J. Phys. D: Appl. Phys., vol. 8, pp. 1585-1594, 1975.

[19] R.S. Becker, Theory and interpretation of fluorescence and phosphorescence, Wiley interscience Ed., 1970.

[20] B. Ranby and J.F. Rabek, Photodegradation, photo-oxydation and photostabilization of polymers: principles and applications, Wiley Interscience Publication, 1975.

[21] C.J. Delbecq, Y. Toyozawa, and P. Yuster, "Tunneling recombination of trapped electrons and holes in $\mathrm{KCl}: \mathrm{AgCl}$ and KCl:TCl," Phys. Rev. B, vol. 9, pp. 4497-4505, 1974. 
[22] P. Butlers, I. Tale, J. Pospisil, and S. Nespurek, "Self-trapping of charge carriers in polymers: A comparative study of poly(p-phenylene) and poly(n-vinylcarbazole)," Prog. Coll. Polym. Sci., vol. 78, pp. 93-96, 1988.

[23] P. Cordier, J.F. Delouis, F. Kieffer, C. Lapersonne, and J. Rigaut, "Etude cinétique de la luminescence différée isotherme d'un verre organique après impulsion d'électrons accélérés," C. R. Hebd. Acad. Sci. C, vol. 279, pp. 589-591, 1974.

[24] A. Charlesby and G.P. Owen, "Luminescence enhancement in irradiated polyethylene," Int. J. Radiat. Phys. Chem., vol. 8, pp. 343-347, 1976.

[25] F. Massines, P. Tiemblo, G. Teyssèdre, and C. Laurent. "On the nature of the luminescence emitted by a polypropylene film after interaction with a cold plasma at low temperature," J. Appl. Phys., vol. 81, pp. 937943, 1997.

[26] Y. Hama, Y. Kimura, M. Tsumura, and N. Omi, "Studies of the recombination of cation-electron pairs by long-range tunneling, as studied by ITL measurement in irradiated polymers," Chem. Phys., vol. 53, pp. 115$122,1980$.

[27] P. Tiemblo, J.M. Gomez-Elvira, G. Teyssedre, F. Massines, and C. Laurent, "Effect of a cold helium plasma at $-180{ }^{\circ} \mathrm{C}$ on polyolefin films - II. The chemiluminescence component," Polym. Degrad. Stab., vol. 64, pp. 67-73, 1999.

[28] W.E. Hagston, "Interpretation of electrophotoluminescence in terms of electron tunneling," J. Phys. C: Solid State Phys., vol. 9, pp. 647-661, 1975.

[29] A.K. Jonscher and A. de Polignac, "The time dependence of luminescence in solids," J. Phys. C: Solid State Phys., vol. 17, pp. 6493-6519, 1984.

[30] Y. Hama, Y. Kimura, M. Tsumura, and N. Omi, "Studies on the recombination of cation-electron pairs by long-range tunneling, as studied by ITL measurement in irradiated polymers," Chem. Phys., vol. 53, pp. 115122,1980

[31] D.J. Huntley, "An explanation of the power-law decay of luminescence," J. Phys.: Condensed Matter, vol. 18, pp. 1359-1365, 2003.

[32] D.V. Balbachas and R.J. Fleming, "Thermally simulated conductivity and luminescence in cross-linked polyethylene," Proc. IEEE Conf. Electr. Insul. Dielec. Phenomena, (CEIDP, Victoria, Canada), pp. 93-98, 1992.

[33] P. Tiemblo, J.M. Gomez-Elvira, G. Teyssedre, and C. Laurent, "Degradative luminescent processes in atactic polypropylene II. Chemiluminescence after a cold He plasma attack at $-180^{\circ} \mathrm{C}$," Polym. Degrad. Stab., vol. 68 , pp. 353-362, 2000.

[34] P. Tiemblo, J.M. Gomez-Elvira, G. Teyssedre, F. Massines, and C. Laurent, "Correlation between polypropylene microstructure, cold plasma interaction and subsequent luminescent emission," Polym. Int., vol. 46, pp. 33-41, 1998.

[35] P. Tiemblo, J.M. Gomez-Elvira, G. Teyssedre, F. Massines, and C. Laurent, "Effect of a cold helium plasma at -180 degrees C on polyolefin films - I. Plasma induced luminescence features of polyethylene and polypropylene," Polym. Degrad. Stab., vol. 64, pp. 59-66, 1999.

[36] M. Duran, F. Massines, G. Teyssedre, and C. Laurent, "Luminescence of plasma-treated polymer surfaces at ambient temperature," Surface \& Coatings Technology, vol. 142, pp. 743-747, 2001.

[37] J. Jonsson, B. Ranby, D. Mary, F. Massines, C. Laurent, and C. Mayoux, "An interpretation of electroluminescence of polyolefins based on the similarity between electro- and plasma-induced luminescence spectra," Proc. IEEE Int. Conf. Solid Dielectr. (ICSD), pp. 701-705, 1995.

[38] J.M. Alison, J. V. Champion, S.J. Dodd, and G.C. Stevens, "Dynamic bipolar charge recombination model for electroluminescence in polymer-based insulation during electrical tree initiation," J. Phys. D: Appl. Phys., vol. 28, pp. 1693-1701, 1995.

[39] M.V. Zamoryanskaya and A.N. Trofimov, "Cathodoluminescence of radiative centers in wide-bandgap materials," Opt. Spectrosc., vol. 115, pp. 79-85, 2013.

[40] M. Pope, H.P. Kallmann, and P. Magnante, "Electroluminescence in organic crystals," J. Chem. Phy., vol. 38, pp. 2042-2043, 1963. 
[41] W.A. Hartman and H.L. Armstrong, "Electroluminescence in organic polymers," J. Appl. Phys., vol. 38, pp. 2393-2395, 1967.

[42] C. Laurent, G. Teyssedre, S. Le Roy, and F. Baudoin, "Charge dynamics and its energetic features in polymeric materials," IEEE Trans. Dielectr. Electr. Insul., vol. 20, pp. 357-381, 2013.

[43] G.A. George, "Characterization of solid polymers by luminescence techniques", Pure Appl. Chem., vol. 57, pp. 945-954, 1985.

[44] K. Jacobson, P. Eriksson, T. Reitberger, and B. Stenberg, "Chemiluminescence as a tool for polyolefin oxidation studies", Adv. Polym. Sci., vol. 169, pp. 151-176, 2004.

[45] K.R. Naveed, L. Wang, H. Yu, R.S. Ullah, M. Haroon, S. Fahad, J. Li, T. Elshaarani, R.U. Khana, and A. Nazira, "Recent progress in the electron paramagnetic resonance study of polymers", Polym. Chem., vol. 9, pp. 33063335, 2018.

[46] I. Blakey and G.A. George, "Simultaneous FTIR emission spectroscopy and chemiluminescence of oxidizing polypropylene: Evidence for alternate chemiluminescence mechanisms", Macromolecules, vol. 34, pp. 18731880, 2001.

[47] J. Rychly J, L. Matisova-Rychla, P. Tiemblo, and J. Gomez-Elvira, "The effect of physical parameters of isotactic polypropylene on its oxidisability measured by chemiluminescence method. Contribution to the spreading phenomenon", Polym. Degrad. Stab., vol. 71, pp. 253-260, 2001.

[48] A. Kron, B. Stenberg, T. Reitberger, and N.C. Billingham, "Chemiluminescence from oxidation of polypropylene: correlation with peroxide concentration", Polym. Degrad. Stab., vol. 53, pp. 119-, 1996.

[49] A. Kron, T. Reitberger, and B. Stenberg, "Luminescence from $\gamma$ - and $\beta$-irradiated HDPE and LLDPE", Polymer International, vol. 42, pp. 131-137, 1997.

[50] E.J. Owen, Editor, Luminescence in Chemistry, Princeton, New Jersey, 1968.

[51] M. Koyanagi, R.J. Zwarich, and L. Goodman, "Phosphorescence Spectrum of acetophenone; an example of pseudo-Jahn-Teller distortion," J. Chem. Phys., vol. 56, pp. 3044-3060, 1972.

[52] P.P.L. Jacques and R.C. Poller, "Fluorescence of polyolefins-2. Use of model compounds to identify fluorescent species in thermally degraded polymers," Eur. Polym. J., vol. 29, pp. 83-89, 1993.

[53] L. Chen, T.D. Huan, C. Wang, and R. Ramprasad, "Unraveling the luminescence signatures of chemical defects in polyethylene," J. Chem. Phys., vol. 143, p. 124907, 2015.

[54] G. Tardieu, G. Teyssedre, and C. Laurent, "Role of additives as recombination centres in polyethylene materials as probed by luminescence techniques," J. Phys. D: Appl. Phys., vol. 35, pp. 40-47, 2002.

[55] G. Teyssedre and C. Laurent, "Semi-quantitative analysis of photoluminescence in thermoelectrically aged cables: I-Identification of optical signatures," IEEE Trans. Dielectr. Electr. Insul., vol. 16, pp. 1180-1188, 2009.

[56] G. Teyssedre, C. Laurent, and G.C. Montanari, "Semi-quantitative analysis of photoluminescence in thermoelectrically aged cables: II-Analysis of a population of cables," IEEE Trans. Dielectr. Electr. Insul., vol. 16, pp. 1189-1198, 2009.

[57] G. Teyssedre, C. Laurent, A. Campus, U.H. Nilsson, and G.C. Montanari, "Antioxidant and its reaction products as charge trapping centres in crosslinked polyethylene," Proc. IEEE Int. Conf. on Electrical Insulation and Dielectric Phenomena (CEIDP, Albuquerque, USA), pp. 96-99, 2003.

[58] G. Teyssedre, G.C. Montanari, C. Laurent, A. Campus, and U.H. Nilsson, "From LDPE to XLPE: investigating the change of electrical properties. Part 2: Luminescence," IEEE Trans. Dielectr. Electr. Insul., vol. 12, pp. 447454, 2005.

[59] F. Peruzzotti, L. Martinotto, and M. Del Brenna, "Cable, in particular for transport or distribution of electrical energy and insulating composition," US Patent 6696154, 2004.

[60] G. Teyssedre, G. Tardieu, D. Mary, and C. Laurent, "AC and DC electroluminescence in insulating polymers and implication for electrical ageing," J. Phys. D: Appl. Phys., vol. 34,pp. 2220-2229, 2001.

[61] C. Laurent, F. Massines, C. Mayoux, D.M. Ryder and C. Olliff, "Comparison between photo- and electroinduced luminescence spectra of polyethylene," Proc. IEEE Int. Conf. on Electrical Insulation and Dielectric Phenomena (CEIDP, Virginia Beach, USA), pp. 95-96, 1995. 
[62] N.S. Allen, J. Homer and J.E. McKellar, "Origin and role of the luminescent species in the photo-oxidation of commercial polypropylene," J. Appl. Polym. Sci., vol. 21, pp. 2261-2267, 1977.

[63] E.J. Bowen, Luminescence in Chemistry (Toronto: Van Nostrand), 1968.

[64] R.S. Becker (Ed.) Theory and Interpretation of Fluorescence and Phosphorescence (New York: Wiley Interscience), 1968.

[65] I. Williams and D.H. Fleming, Spectroscopic methods in organic chemistry, McGraw Hill, London, 1973.

[66] W. Klopffer, "Luminescent groups in very weakly degraded polystyrene," Eur. Polym. J., vol. 11, pp. 203208, 1975

[67] A.A. Lamola, "Lowest $\pi-\pi^{*}$ triplet state of acetophenone," J. Chem. Phys., vol. 47, pp. 4810-4816, 1967.

[68] C. Laurent, A. Campus et al., "Evaluation and modelling of thermoelectric ageing of XLPE insulated power cables: the ARTEMIS outcome," Proc. 6th Int. Conf. on Insulated Power Cables (JiCable, Versailles), pp. 525533,2003

[69] H. Herman, J. Thomas and G. Stevens, "Spectroscopic and chemometrics analysis of cable condition in the Artemis program," Proc. IEEE Internat. Conf. Solid Dielectrics (ICSD, Toulouse, France), pp. 623-627, 2004.

[70] S. Le Roy, G. Teyssedre, and C. Laurent, "Charge transport and dissipative processes in insulating polymers: experiments and model," IEEE Trans. Dielectr. Electr. Insul., vol. 12, pp. 644-654, 2005.

[71] See for example J.I. Pankove (Ed.) Electroluminescence, Springer, New York, 1977.

[72] L. Sanche, "Electronic aging and related electron interactions in thin-film dielectrics," IEEE Trans. Electr. Insul., vol. 28, pp. 789-819, 1993.

[73] H.R. Zeller, P. Pfluger and J. Bernasconi, "High-mobility states and dielectric breakdown in polymeric dielectrics," IEEE Trans. Electr. Insul, vol. 19, pp. 200-204, 1984.

[74] C. Laurent, G. Teyssedre, and G.C. Montanari, "Time-resolved space charge and electroluminescence measurements in polyethylene under AC stress," IEEE Trans. Dielectr. Electr. Insul., vol. 11, pp. 554-560, 2004.

[75] G. Teyssedre, L. Cisse, D. Mary, and C. Laurent, "Identification of the components of the electroluminescence spectrum of PE excited in uniform fields," IEEE Trans. Dielectr. Electr. Insul., vol. 6, pp. 11-19, 1999.

[76] B. Qiao, C. Laurent, and G. Teyssedre, "Electroluminescence and cathodoluminescence from polyethylene and polypropylene films: spectra reconstruction from elementary components and underlying mechanisms," J. Appl. Phys., vol. 119, p. 024103, 2016.

[77] G. Teyssedre and C. Laurent, "Evidence of hot electron-induced chemical degradation in electroluminescence spectra of polyethylene," J. Appl. Phys., vol. 103, p. 046107, 2008.

[78] B. Qiao, C. Laurent, and G. Teyssedre, "Evidence of exciton formation in thin polypropylene films under AC and DC fields and relationship to electrical degradation," Proc. Int. Symp. Electr. Insul. Materials (ISEIM, Niigata City, Japan), pp. 81-84, 2014.

[79] C. Laurent and G. Teyssedre, "Hot electron and partial-discharge induced aging of polymers," Nucl. Instr. and Meth. in Phys. Res. B, vol. 208, pp. 442-447, 2003.

[80] L. Sanche, IEEE Trans. Dielectr. Electr. Insul., "Nanoscopic aspects of electronic aging in dielectrics," vol. 4, pp. 507-543, 1997.

[81] G. Teyssedre, D. Mary, J.L. Augé, and C. Laurent, "Dependence of electroluminescence intensity and spectral distribution on ageing time in polyethylene naphthalate as modelled by space charge - modified internal field," J. Phys. D: Appl. Phys., vol. 32, pp. 2296-2305, 1999.

[82] J. Goldstein, D. Newbury, D. Joy, C. Lyman, P. Echlin, L. Sawyer, and J. Michael, Scanning Electron Microscopy and X Ray Microanalysis (Kluwer Academic/Plenum Publishers, New York), 2003.

[83] J.W. Allen, "Impact processes in electroluminescence," J. Lumin., vol. 48, pp. 18-22, 1991.

[84] L. Sanche, "Dissociative attachment and surface reactions induced by low-energy electrons," J. Vac. Sci. Technol. B, vol. 10, pp. 196-200, 1992. 
[85] D. Ceresoli, M.C. Righi, E. Tosatti, S. Scandolo, G. Santoro and S. Serra "Exciton self-trapping in bulk polyethylene," J. Phys.: Condens. Mat., vol. 17, p. 4621, 2005.

[86] C.R. Bealing, and R. Ramprasad, "An atomistic description of the high-field degradation of dielectric polyethylene," J. Chem. Phys., vol. 139, p. 1749043, 2013.

[87] D. Ceresoli, E. Tosatti, S. Scandolo, G. Santoro and S. Serra "Trapping of excitons at chemical defects in polyethylene," J. Chem. Phys., vol. 121, pp. 6478-6484, 2004.

[88] D. Mary and D. Malec, "Electroluminescence measurements to detect accumulated charge at the electrode-insulator interface," IEEE Trans. Dielectr. Electr. Insul., vol. 8, pp. 771-775, 2001.

[89] A. See, J.C. Fothergill, L.A. Dissado, G.C. Stevens, G.C. Montanari, C. Laurent, and G. Teyssedre, "Evaluation of the onset of space charge and electroluminescence as a marker for cross-linked polyethylene ageing," 4th Int. Conf. on Electric Charges in non-Conductive Materials, (CSC, Tours, France), pp. 185-188, 2001.

[90] F. Gullo, C. Villeneuve-Faure, S. Le Roy, C. Laurent, G. Teyssedre, T. Christen, and H. Hillborg, "Impact of press-molding process on chemical, structural, and dielectric properties of insulating polymers," Proc. 8th Int. Symp. Electr. Insul. Materials (ISEIM, Toyohashi City, Japan), pp. 69-72, 2017.

[91] G. Teyssedre, J. Menegotto, and C. Laurent. "Temperature dependence of the photoluminescence in Poly(ethylene terephthalate)," Polymer, vol. 42, pp. 8207-8216, 2001.

[92] Y. Li and T. Takada, "Space charge distribution in multi-ply LDPE," Proc. Conf. Electrical Insulation and Dielectric Phenomena (CEIDP, Victoria, Canada), pp. 397-402, 1992.

[93] H. Ghorbani, F. Abid, H. Edin and M. Saltzer, "Effect of heat-treatment and sample preparation on physical properties of XLPE DC cable insulation material," IEEE Trans. Dielectr. Electr. Insul., vol. 23, pp. 2508-2516, 2016.

[94] Y. Ohki, N. Hirai, K. Kobayashi, R. Minami, M. Okashita, and T. Maeno, "Effects of byproducts of crosslinking agent on space charge formation in polyethylene - comparison between acetophenone and $\alpha$ methylstyrene," Proc. IEEE Conf. Electrical Insulation and Dielectric Phenomena (CEIDP, Victoria, Canada), pp. 535-538, 2000.

[95] J. Kalinowski, Organic Electroluminescent Materials and Devices, Ed. S Miyata and H R Nalwa (New York: Gordon and Breach), Chap. 1, 1997. 